\title{
Surface Wave, Internal Wave, and Source Motion Effects on Matched Field Processing in a Shallow Water Waveguide
} by

\author{
John Russell Daugherty \\ B.S., United States Naval Academy (1981) \\ Submitted in partial fulfillment of the \\ requirements for the degree of \\ OCEAN ENGINEER \\ and \\ MASTER OF SCIENCE IN OCEAN ENGINEERING \\ at the \\ MASSACHUSETTS INSTITUTE OF TECHNOLOGY \\ and the \\ WOODS HOLE OCEANOGRAPHIC INSTITUTION
}

August 1989

(C) John Russell Daugherty, 1989

The author hereby grants to MIT and WHOI permission to reproduce and to distribute copies of this thesis document in whole or in part.

Signature of Author

Joint Program in Oceanographic Engineering Massachusetts Institute of Technology Woods Hole Oceanographic Institution August 11, 1989

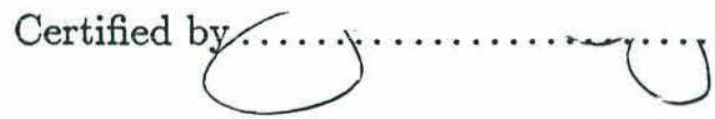

Dr. James F. Lynch Woods Hole Oceanographic Institution Thesis Supervisor

Certified by ...........

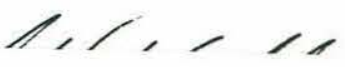

Accepted by .

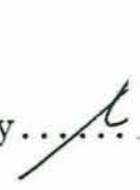

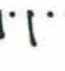

Aassachuse

Dr. Arthur B. Baggeroer

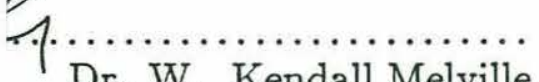

Dr. W. Kendall Melville

Chairman,Joint Committee for Oceanographic Engineering Massachusetts Institute of Technology/Woods Hole Oceanographic Institution 


\title{
Surface Wave, Internal Wave, and Source Motion Effects on Matched Field Processing in a Shallow Water Waveguide \\ by John Russell Daugherty
}

\author{
Submitted to the Massachusetts Institute of Technology/ \\ Woods Hole Oceanographic Institution \\ Joint Program in Oceanographic Engineering \\ on August 11, 1989, in partial fulfillment of the \\ requirements for the degree of \\ Ocean Engineer \\ and \\ Master of Science in Ocean Engineering
}

\begin{abstract}
Given well known environmental conditions, matched field processing has been shown to be a promising signal processing technique for the localization of acoustic sources. However, when environmental data are incomplete or inaccurate, a 'mismatch' occurs between the measured field and model field which can lead to a severe degradation of the localization estimator. We investigate the possible mismatch effects of surface and internal waves on matched field processing in a shallow water waveguide. We utilize a modified ray theory, based on the work of Tindle, to calculate the acoustic pressure field. This allows us to simply incorporate range dependent environmental conditions as well as to generalize our work to deeper waveguides. In general, the conventional (Bartlett) matched field beamformer does not provide sufficient resolution to unambiguously locate a source, even in a perfectly matched environment. The maximum likelihood method (MLM) matched field beamformer has much better resolution but is extremely susceptible to mismatch. The mismatch due to surface roughness can result in a large reduction of the estimator peak. Part, but not all, of the peak can be regained by 1)using a model which includes incomplete reflection at the surface based on actual sea surface statistics and 2) short time averaging of the measured signal, with times on the order of the period of the surface waves. Mismatch due to internal waves can also result in a large degradation of the estimator. Averaging over the same time period as surface waves provides little improvement and leads one to surmise that internal waves may be a limiting constraint on matched field processing. Finally, we combine the surface and internal wave fields with a slowly moving source. This example highlights the necessity for the development of a beamformer which has a broader mainlobe while maintaining adequate sidelobe suppression, and we address this issue by looking at two such beamformers.
\end{abstract}

Thesis Supervisor: Dr. James F. Lynch

Woods Hole Oceanographic Institution 


\section{Acknowledgements}

I take this opportunity to thank the following people who have enabled me to complete my research at MIT/WHOI.

- For invaluable guidance and encouragement, my thesis advisor, Dr. Jim Lynch.

- For the many hours of computer assistance, Arthur Newhall.

- For their time, assistance and computer algorithms, particularly the SAFARI code, Dr. Arthur Baggeroer and Dr. Henrik Schmidt.

- For her many helpful comments while proofing the thesis, Dr. Alex Tolstoy.

- For many of the interesting graphics, Betsy Pratt and Roger Goldsmith.

- For the countless hours of discussion on topics both relavent and inane, my fellow Joint Program cohorts.

- For providing the opportunity as well as the financial backing, the United States Navy.

- And last (but always first), my wife Linda, for her enduring love and support. 


\section{Contents}

1 Introduction $\quad 9$

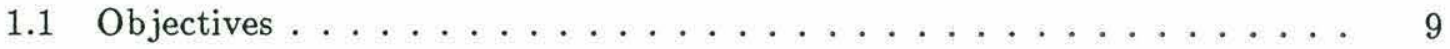

1.2 Basic Waveguide Model . . . . . . . . . . . . . . . . . . 10

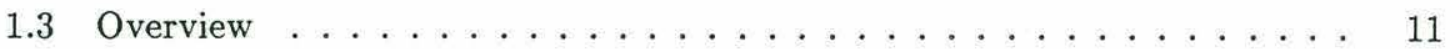

2 Components of the Analysis $\quad 12$

2.1 Ray Theory with Beam Displacement . . . . . . . . . . . . 12

2.2 Scattering from Surface Waves . . . . . . . . . . . . 16

2.2 .1 Surface Wave Model . . . . . . . . . . . . . . . . . 16

2.2 .2 Surface Scattering Model . . . . . . . . . . . . . . . 19

2.3 Internal Wave Model . . . . . . . . . . . . . . . . . . 23

2.4 Matched Field Processing . . . . . . . . . . . . . . 31

2.4.1 Bartlett Beamformer . . . . . . . . . . . . . . 31

2.4.2 Maximum Likelihood Beamformer . . . . . . . . . . . . . . 33

2.4.3 Maximum Constraint Beamformer . . . . . . . . . . . . . . 34

2.4.4 Variable Coefficient Likelihood Beamformer . . . . . . . . . . . 37

3 Analysis and Results $\quad 43$

3.1 Estimation of the Covariance Matrix . . . . . . . . . . . . 43

3.2 Surface Wave Effects . . . . . . . . . . . . . . . . . . 44

3.2 .1 Analysis . . . . . . . . . . . . . . . . . . 44

3.2.2 Comparison of BE and MLM Processors in an Ideal Case . . . . 44

3.2.3 Effects of increasing wave heights on the MLM estimator . . . . 45 
3.2.4 Counteracting surface wave effects ........... 50

3.3 Internal Wave Effects . . . . . . . . . . . . . . 53

3.3 .1 Analysis . . . . . . . . . . . . . . 53

3.3.2 Effects of single frequency internal wave on the MLM estimator . . 53

3.3.3 Counteracting internal wave effects . . . . . . . . . 55

3.4 Moving Source Effects . . . . . . . . . . . . . . . . 59

3.4 .1 Analysis . . . . . . . . . . . . . . . . . . 59

3.4.2 Results from Moving Source Effects . . . . . . . . . . . 59

3.5 Combined Effects and Sub-optimal Processors . . . . . . . . . . . . . 59

3.5 .1 Analysis . . . . . . . . . . . . . . . 59

3.5.2 Results from Combined Effects . . . . . . . . . . . 63

4 Discussion and Conclusions $\quad 68$

$\begin{array}{ll}\text { A Derivation of the Surface Scattering Integral } & \mathbf{7 0}\end{array}$

A.1 Helmholtz Integral Equation . . . . . . . . . . . . . . . 70

A.2 Evaluation of Helmholtz Integral Equation . . . . . . . . . . . 71 


\section{List of Figures}

2-1 A typical ray path with beam displacement included. The expanded view is an actual pressure field generated by SAFARI showing the displacement of the beam at the interface. . . . . . . . . . . . . 13

2-2 Comparison of transmission loss curves . . . . . . . . . . . . . 15

2-3 A wavenumber spectrum of the ocean waves . . . . . . . . . 16

2-4 (a) A cross section of the model surface and (b) the corresponding surface height probability density function based on summing discete frequencies. 18

2-5 Idealized reflection from a rough sea surface in the specular direction. . . 19

2-6 Comparison of the reflection coefficient numerically calculated for the model surface vs. the theoretical Gaussian rough surface. . . . . . . . . . . 22

2-7 Comparison of transmission loss curves based on ray theory with beam displacement (dashed line) and the actual solution (solid line) including the effect of a random rough surface with $1 \mathrm{~m}$ rms wave height. . . . . . . 23

2-8 An acoustic record of an internal wave field. The period of the waves are doppler shifted by the ship's speed of about 2.5 knots. . . . . . . . 25

2-9 (a) A typical ray path through a shallow water internal wave (b) An expanded view showing the intensity fluctuations of the ray. . . . . . . 26

2-10 Comparison of transmission loss curves based on ray theory with beam displacement (dashed line) and the actual solution (solid line) for the three layer internal wave model. . . . . . . . . . . . . . . . . . 28

2-11 Total intensity loss across the layer using an approximation of total transmission (dashed line) and the actual transmission coefficient (solid line). Both include spreading losses. . . . . . . . . . . . . . 30 
2-12 Plane wave ambiguity functions for a $\mathrm{BE}$ processor(dashed line) and an MLM processor(solid line) given an SNR of (a)-10 dB and (b) $10 \mathrm{~dB}$. . . . 35

2-13 Plane wave ambiguity function for a BE processor(dashed line), an MLM processor(solid line), and an MCM processor(dash-dotted line) in the case of (a) no mismatch and (b) a mismatch caused by plane wave assumption with source close to array. . . . . . . . . . . . . . 38

2-14 (a)Output of VCLM processor vs. correlation coefficient for exponent values of 1.0, 1.5, and 2.0. (b) Difference between MLM output and VCLM output vs. correlation coefficient for exponent values of 1.5 and 2.0 . . . 40

2-15 Plane wave ambiguity function for a BE processor(dashed line), an MLM processor(solid line), and an VCLM processor(dash-dotted line) in the case of (a) a perfect match and (b) a mismatch caused by plane wave assumption with source close to array. . . . . . . . . . . . . . . . . . . 42

3-1 Ambiguity surface using a $\mathrm{BE}$ processor in an ideal case. True source location is at a range of $4 \mathrm{~km}$ and depth of $54 \mathrm{~m} . \ldots \ldots$. . . . . . . 46

3-2 Ambiguity surface using an MLM processor in an ideal case. True source location is at a range of $4 \mathrm{~km}$ and depth of $54 \mathrm{~m} . \ldots . . . \ldots 47$

3-3 MLM ambiguity function for mismatched surface condition of $0.5 \mathrm{~m}$ rms wave height. True source location is at a range of $4 \mathrm{~km}$ and depth of $54 \mathrm{~m}$. 48

3-4 MLM ambiguity function for mismatched surface condition of $1.0 \mathrm{~m} \mathrm{rms}$ wave height. True source location is at a range of $4 \mathrm{~km}$ and depth of $54 \mathrm{~m}$.

3-5 MLM ambiguity function for matched surface condition of $0.5 \mathrm{~m}$ rms wave height. True source location is at a range of $4 \mathrm{~km}$ and depth of $54 \mathrm{~m}$. . . . . 51

3-6 MLM ambiguity function for matched surface condition of $1.0 \mathrm{~m}$ rms wave height. True source location is at a range of $4 \mathrm{~km}$ and depth of $54 \mathrm{~m}$. . . . 52

3-7 The effect on peak-to-background ratios for various surface wave height models vs. actual wave height $\ldots \ldots \ldots \ldots \ldots$. . . . . . . 54

3-8 MLM ambiguity function for internal wave condition of $0.0 \mathrm{~m}$ amplitude internal wave height. True source location is at a range of $4 \mathrm{~km}$ and depth

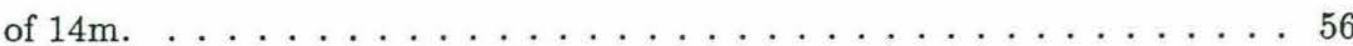


3-9 MLM ambiguity function for internal wave condition of $2.0 \mathrm{~m}$ amplitude internal wave height. True source location is at a range of $4 \mathrm{~km}$ and depth

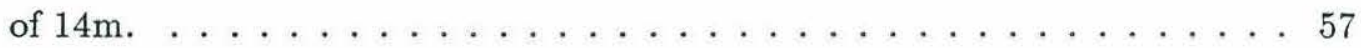

3-10 PBR as a function of phase estimation error for internal wave. . . . . . 58

3-11 MLM ambiguity function for moving source effects. True source location is at a range of $4 \mathrm{~km}$ and depth of $14 \mathrm{~m}$ at $\mathrm{t}=0.0$, speed 5 knots toward receiver. $\quad 60$

3-12 MLM ambiguity function for moving source effects. True source location is at a range of $4 \mathrm{~km}$ and depth of $14 \mathrm{~m}$ at $\mathrm{t}=0.0$, speed 10 knots toward receiver. ............................... 61

3-13 MLM ambiguity function for moving source effects. True source location is at a range of $4 \mathrm{~km}$ and depth of $14 \mathrm{~m}$ at $\mathrm{t}=0.0$, speed 15 knots toward

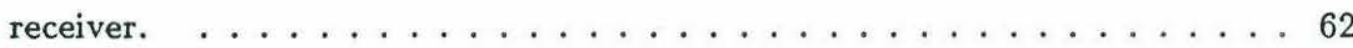

3-14 MLM ambiguity function for combined effects. True source location is at a range of $4 \mathrm{~km}$ and depth of $14 \mathrm{~m}$ at $\mathrm{t}=0.0$, speed $5 \mathrm{~km} / \mathrm{hr}$ toward receiver. . 65

3-15 MCM ambiguity function for combined effects. True source location is at a range of $4 \mathrm{~km}$ and depth of $14 \mathrm{~m}$ at $\mathrm{t}=0.0$, speed $5 \mathrm{~km} / \mathrm{hr}$ toward receiver. $\quad 66$

3-16 VCLM ambiguity function for combined effects. True source location is at a range of $4 \mathrm{~km}$ and depth of $14 \mathrm{~m}$ at $\mathrm{t}=0.0$, speed $5 \mathrm{~km} / \mathrm{hr}$ toward receiver. $\quad 67$

A-1 Calculation of pressure field in a volume V. . . . . . . . 71

A-2 Scattering from a rough surface . . . . . . . . . . 72 


\section{Chapter 1}

\section{Introduction}

\section{$1.1 \quad$ Objectives}

Traditionally, source localization has relied on the processing of assumed plane wavefronts to estimate bearing and, in restricted situations, approximate range. In reality, the ocean is extremely complex and the assumption of plane waves in the processing scheme can lead to severe degradation of the estimate. Recently, with the advent of more powerful computers, optimal signal processing techniques have been developed which can use the complexity of the ocean's structure to actually improve source localization. One such technique, which has recently received much attention, is that of matched field processing.

Matched field processing involves the correlation of the actual acoustic pressure field measured at a receiver array with a predicted field based on a postulated source position and an assumed ocean model. A high degree of correlation, or 'match', between the measured field and the predicted field indicates a likely source location. Thus, an increased complexity of the ocean's structure provides a greater variability of the acoustic fields, which aids the estimation procedure. However, matched field processing is also predicated on having sufficient knowledge of the ocean's structure, which allows accurate prediction of the acoustic pressure field. When the environmental data used to calculate the predicted fields are inaccurate or incomplete, a 'mismatch' occurs between measured data and the predicted pressure field, leading to errors in the estimator.

There have been several studies in the last few years investigating the sensitivity 
of matched field processing to errors in the model. For example, DelBalzo et.al.(1988) addressed water depth mismatches in a shallow water channel, Feuillade et. al.(1989) studied geoacoustic parameter variability, and Tolstoy(1989) investigated low frequency sensitivity to sound speed profile mismatch. On the whole, they have dealt only with deterministic mismatches. We turn our investigation here to the following issues:

- Environmental effects of surface and internal waves which, in general, must be treated as random processes. As such, wave effects prevent convergence to a single model and result in the degradation of the estimator.

- Moving source effects which, in general, prevent the correlation to a single grid point and, therefore, degrade the estimate.

- A combination of surface and internal waves with a slowly moving source to obtain a more realistic look at the robustness of the various matched field processors.

- The development of more robust matched field processors.

\subsection{Basic Waveguide Model}

In the investigation of surface wave effects, we consider the localization of a $150 \mathrm{~Hz}$ source in a simple two fluid Pekeris model, which is a good first approximation to many shallow water situations. The density ratio of the sediment to water is $\rho_{\text {bottom }} / \rho_{\text {water }}=$ 1.772. The speed of sound is $c_{\text {water }}=1500 \mathrm{~m} / \mathrm{s}$ in the water layer and $c_{\text {bottom }}=1621.62$ $\mathrm{m} / \mathrm{s}$ in the sediment halfspace. The depth of the channel is $H=100$ meters. The sediment half space is modeled as a fluid. For simplicity, attenuation is neglected. This is the same shallow water model used by Fizell (1987) and DelBalzo et. al.(1988).

Our model is extended to include a two layer water channel over a sediment half space for the investigation of internal wave effects. The two layer water channel is widely used in oceanography as a first approximation, for it allows a resonable theoretical explication without inordinate complications. The two layer stratification results in the formation of an internal wave which propagates along the interface of the discontinuity. The water layers are modeled as a warm surface layer over a colder bottom layer, i.e. $c^{\prime}{ }_{\text {water }}=1505$ 
$\mathrm{m} / \mathrm{s} ; c_{\text {water }}=1500 \mathrm{~m} / \mathrm{s}$. The density in the warm layer has decreased by $.001 \mathrm{gm} / \mathrm{cc}$. All other channel parameters remain the same. This same model is used when the surface wave and internal wave fields are combined with a moving source.

\subsection{Overview}

This thesis is organized as follows. In Chapter 2, we discuss the fundamentals of our analysis. We begin with a brief discussion of ray theory and show the necessity of including 'beam displacements' in order to accurately model the acoustic pressure field in shallow water at low frequency. Next, we present our surface wave model, which uses an analytical spectrum with a Gaussian sea surface. The assumptions for the surface reflection calculations are also included in this section. In the next section, we look at the effects of single frequency spectral components of a two layer internal wave model on sound transmission. Following that, we provide some background on the signal processing techniques of matched field processing which we use in our study. Included in this section is a presentation of more robust techniques of matched field processing which we incorporate. In Chapter 3, we present our detailed analysis of surface wave, internal wave, and source motion effects on matched field processing. A summary of the work and a discussion of areas of future research are included in Chapter 4. 


\section{Chapter 2}

\section{Components of the Analysis}

\subsection{Ray Theory with Beam Displacement}

In this thesis, we use ray theory to describe the acoustic field since it allows us to easily incorporate realistic boundary conditions and to extend our work staightforwardly to deeper waveguides. However, the use of ray theory may be questioned since it is generally thought to be restricted to high frequencies and, conversely, early studies indicate that matched field localization is most effective at low frequencies (Baggeroer, pers. comm.). This situation is easily resolved by the incorporation of 'beam displacement' into the ray theory calculations. The beam displacement is an effective horizontal displacement along an interface of a bounded plane wave (ray) upon reflection from that surface. Tindle has shown that the ray method with beam displacement is capable of describing even low frequency acoustic fields in certain shallow water waveguides to a high degree of accuracy (Tindle and Bold,1981; Tindle,1983).

A typical ray path in an isovelocity shallow water waveguide is shown in Figure 21. The source and receiver are located at depths of $Z_{o}$ and $Z_{r}$ respectively. The beam displacement, indicated by $\Delta$, occurs at each bottom interaction. An expanded view of the ray path at the water-sediment interface was generated using a fast field computer code known as SAFARI (Schmidt,1987). This view of the pressure field clearly shows the reflected beam displaced horizontally. This results from the excitation of the lateral wave when the incident wave is just below the critical angle. The reflected field is then 

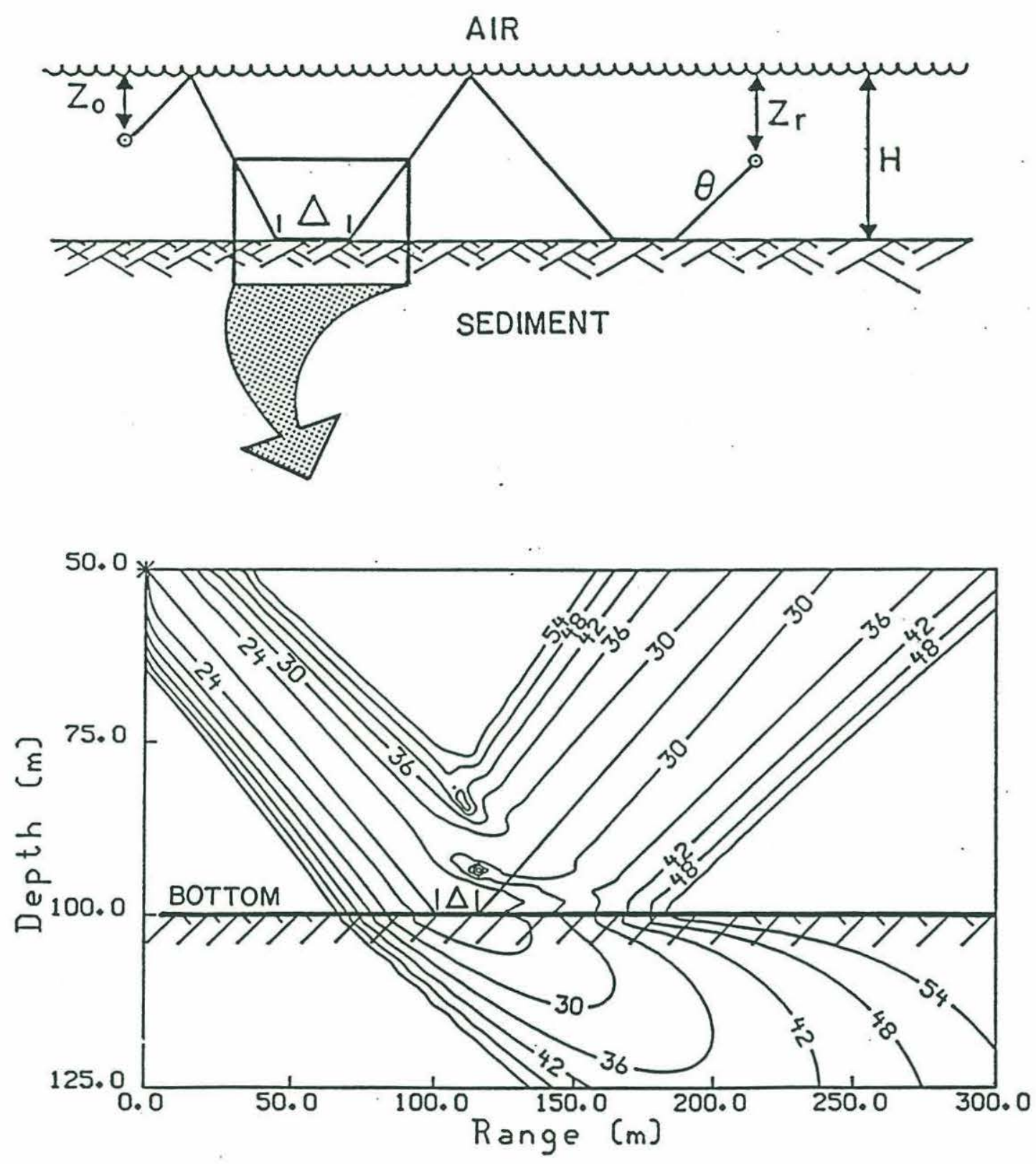

Figure 2-1: A typical ray path with beam displacement included. The expanded view is an actual pressure field generated by SAFARI showing the displacement of the beam at the interface. 
composed of contributions from both the specular beam and the lateral field, causing the apparent horizontal displacement of the beam. Further examples and discussion of beam displacement can be found in Schmidt and Jensen (1985) and Jensen and Schmidt (1987).

For the simple case of a beam incident from one uniform half-space with sound speed $c_{1}$ and density $\rho_{1}$ onto a second uniform half-space with sound speed $c_{2}>c_{1}$ and density $\rho_{2}$, the reflection coefficient for total internally reflected rays ${ }^{1}$ may be written

$$
R(\theta)=\left(\rho_{2} \gamma_{1}-i \rho_{1} \gamma_{2}\right) /\left(\rho_{2} \gamma_{1}+i \rho_{1} \gamma_{2}\right)
$$

where $\theta$ is the grazing angle, and $\gamma_{1}\left(=\frac{\omega}{c_{1}} \sin \theta\right)$ and $\gamma_{2}\left(=\frac{\omega}{c_{1}} \sqrt{\cos ^{2} \theta-\left(c_{1} / c_{2}\right)^{2}}\right)$ are the vertical wavenumbers in media 1 and 2 , respectively. The phase of the reflection coefficient is given by

$$
\phi=-2 \tan ^{-1}\left(\rho_{1} \gamma_{2} / \rho_{2} \gamma_{1}\right)
$$

The lateral displacement of the ray, as shown by Brekhovskikh(1960), is

$$
\Delta=\partial \phi / \partial k_{r}=2 k_{r} \rho_{1} \rho_{2}\left(\gamma_{1}^{2}+\gamma_{2}^{2}\right) /\left[\gamma_{1} \gamma_{2}\left(\rho_{1}^{2} \gamma_{2}^{2}+\rho_{2}^{2} \gamma_{1}^{2}\right)\right]
$$

where $k_{r}\left(=\frac{\omega}{c_{1}} \cos \theta\right)$ is the horizontal wavenumber. The group time displacement, $\tau$, corresponding to the beam displacement was given by Zhang (1975) as

$$
\tau=\partial \phi / \partial \omega=2 \omega \rho_{1} \rho_{2}\left(\gamma_{1}^{2} / c_{2}^{2}+\gamma_{2}^{2} / c_{1}^{2}\right) /\left[\gamma_{1} \gamma_{2}\left(\rho_{1}^{2} \gamma_{2}^{2}+\rho_{2}^{2} \gamma_{1}^{2}\right)\right]
$$

Therefore, the apparent speed of the horizontal beam displacement in the bottom is $\Delta / \tau=c_{1} / \cos \theta$.

The pressure field measured by a vertical array of $M$ sensors can now be constructed by coherently summing the contributions of all the totally internally reflected eigenrays (i.e. all the rays leaving the source which pass through the receiver),

$$
P\left(\mathbf{z}_{m}, \omega \mid \mathbf{r}\right)=\sum_{l=1}^{L} A_{l m}(\mathbf{r}) e^{j \omega T_{l m}(\mathbf{r})}
$$

The factor $A_{l m}(\mathbf{r})$ is the complex amplitude of the $l^{\text {th }}$ eigenray between a source located at $\mathbf{r}\left(r_{o}, z_{o}\right)$ and the $m^{\text {th }}$ element of the receiver. This factor incorporates effects from (nonrough) surface and bottom interactions as well as geometric spreading. The propagation

\footnotetext{
${ }^{1}$ In our study, we will consider only rays which have total internal reflection. Rays which have a reflection coefficient less than unity are taken to be attenuated by the multiple bottom reflections prior to reaching the receiver and are thus ignored.
} 
delay, including the effect of the beam displacement, along the $l^{\text {th }}$ eigenpath to the $m^{\text {th }}$ sensor is denoted $T_{l m}(\mathbf{r})$. We have assumed that the signal has a narrow bandwidth so that the reflection coefficient, phase factor, and beam displacement can be calculated at the center frequency.

\section{TRANSMISSION LOSS}

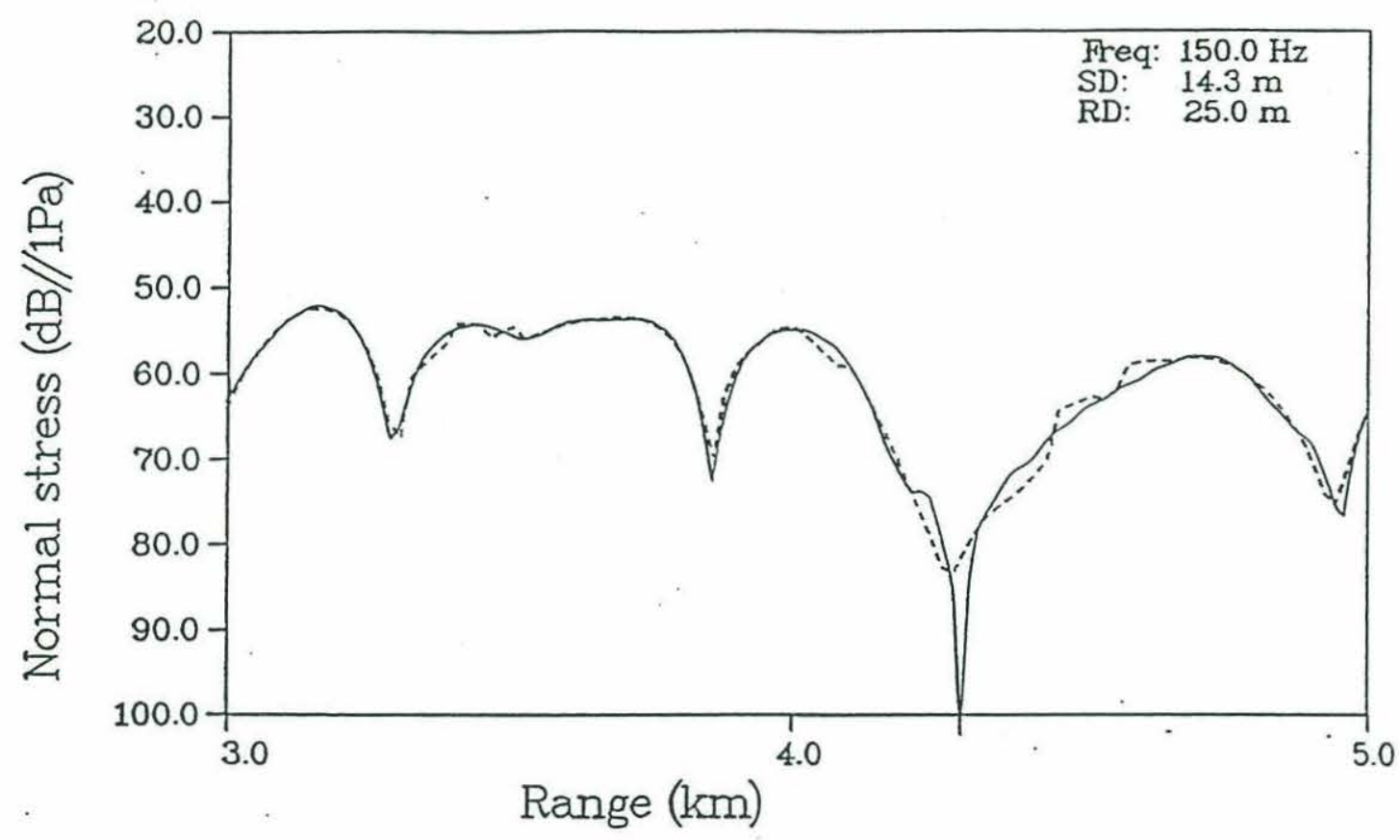

Figure 2-2: Comparison of transmission loss curves based on ray theory with beam displacement (dashed line) and the actual solution (solid line).

Figure 2-2 compares transmission loss curves generated by ray theory with beam displacement with an 'exact' solution generated by SAFARI. The dashed line in Figure 22 is the transmission loss calculated with the ray theory. The solid line is the 'exact' solution. As claimed, the beam displacement method accurately describes the acoustic field. The solutions, as expected, diverge in the vicinity of a caustic, which requires an additional correction to ray theory. Westwood and Tindle(1987) incorporated this correction into a beam displacement algorithm with excellent results. Our work here is limited by the necessity of finding large numbers of eigenrays. The additional caustic corrections, while giving a more accurate acoustic field, are not required to study the 
wave effects on matched field processing, and so have not been included.

\subsection{Scattering from Surface Waves}

\subsubsection{Surface Wave Model}

Surface gravity waves, to a good first approximation, propagate independently of each other, and thus the ocean surface can be characterized by a sum of sinusoidal waveforms. A realization of the surface wave spectrum is obtained by the superposition of many wave trains of different discrete frequencies and with random phase. A typical ocean wavenumber spectrum from Pierson and Stacy (1973) is shown in Figure 2-3.

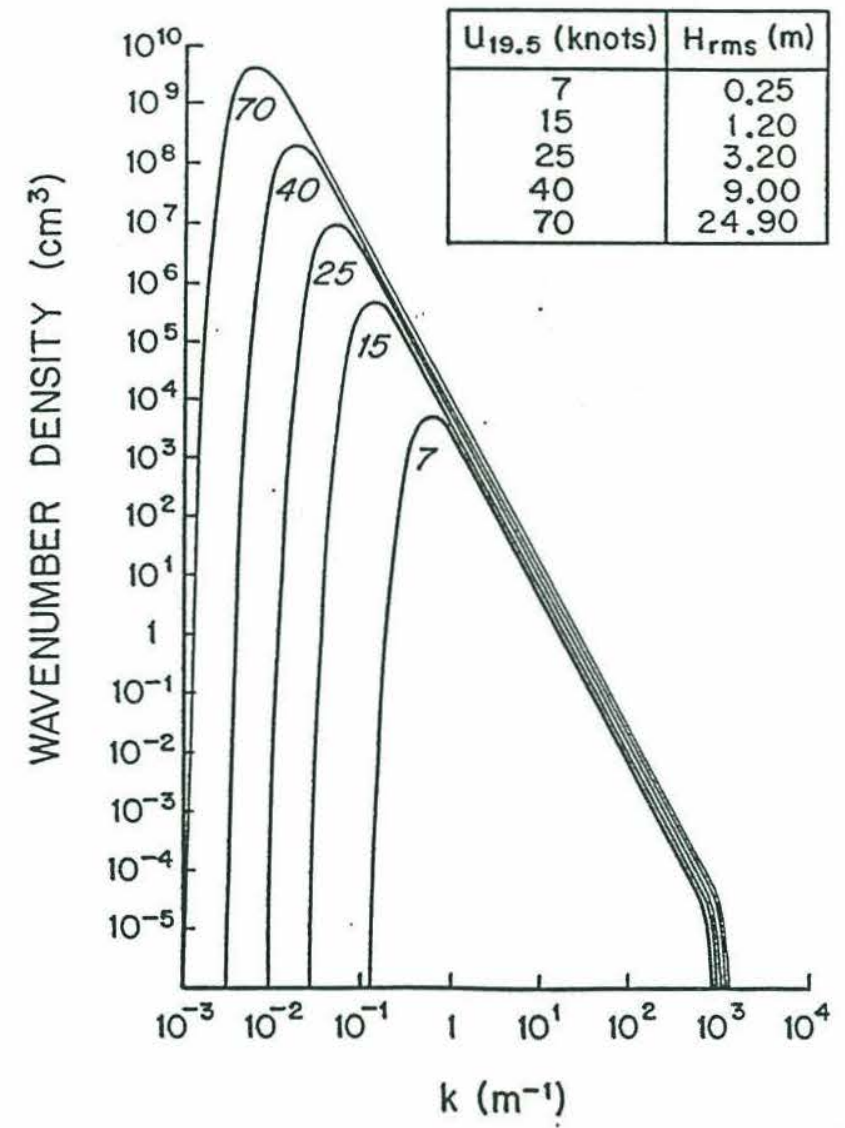

Figure 2-3: A wavenumber spectrum of the ocean waves for wind speeds from 7 to 70 knots. $\mathrm{U}_{19.5}$ is the wind speed measured $19.5 \mathrm{~m}$ above the sea surface. $\mathrm{H}_{r m s}$ is the effective rms waveheight.

If we neglect spray, air bubbles in the water, and breaking waves, the sea surface is 
a single valued function which we denote as $\xi(s, t)$ which is a function of the horizontal displacement $s$ (equal to $\sqrt{x^{2}+y^{2}}$ ) and the time function $t$. Considering only waves traveling on a line between source and receiver, the resultant sea surface displacement from the mean is written in discrete form as

$$
\xi(s, t)=\sum_{n=1}^{\infty} a_{n} \cos \left(k_{n} s+\omega_{n} t+\epsilon_{n}\right)
$$

where $a_{n}$ is the amplitude of the $n^{\text {th }}$ component of the surface wave spectrum which has a spatial wavenumber $k_{n}$ and temporal frequency $\omega_{n}$ and $\epsilon_{n}$ is a phase lag which varies randomly from 0 to $2 \pi$. Using the linearized hydrodynamic equations of motion of a free surface, the wavenumber and frequency are related by the dispersion relationship

$$
\omega_{n}=\sqrt{g k_{n} \tanh k_{n} H}
$$

where $H$ is the depth of the channel.

In this study, we have selected five discete wavelengths ranging from 30 to 80 meters, as shown in Table 2.1, to approximate the continuous spectrum. The amplitudes are for a wind speed range of 7 to 15 knots.

\begin{tabular}{||c|c|c|c||}
\hline $\mathrm{n}$ & $a_{n}(m)$ & $\lambda_{n}(m)$ & $T_{n}(\mathrm{sec})$ \\
\hline 1 & 0.75 & 78.5 & 7.1 \\
\hline 2 & 1.00 & 62.8 & 6.35 \\
\hline 3 & 0.75 & 52.3 & 5.8 \\
\hline 4 & 0.50 & 44.9 & 5.35 \\
\hline 5 & 0.25 & 39.2 & 5.0 \\
\hline
\end{tabular}

Table 2.1: Parameters used in construction of surface wave model

According to experimental data obtained in the ocean (Tung and Huang,1985), the statistical distribution of $\xi$ is very close to Gaussian. Figure 2-4 shows a cross section of the model surface and plots the corresponding surface height probability density function. Superimposed is a Gaussian function with the same variance as the model surface $\left(\sigma^{2}=1\right)$. As seen, even a limited number of discrete frequency components generates a psuedorandom wave surface whose height distribution approaches a Gaussian. 

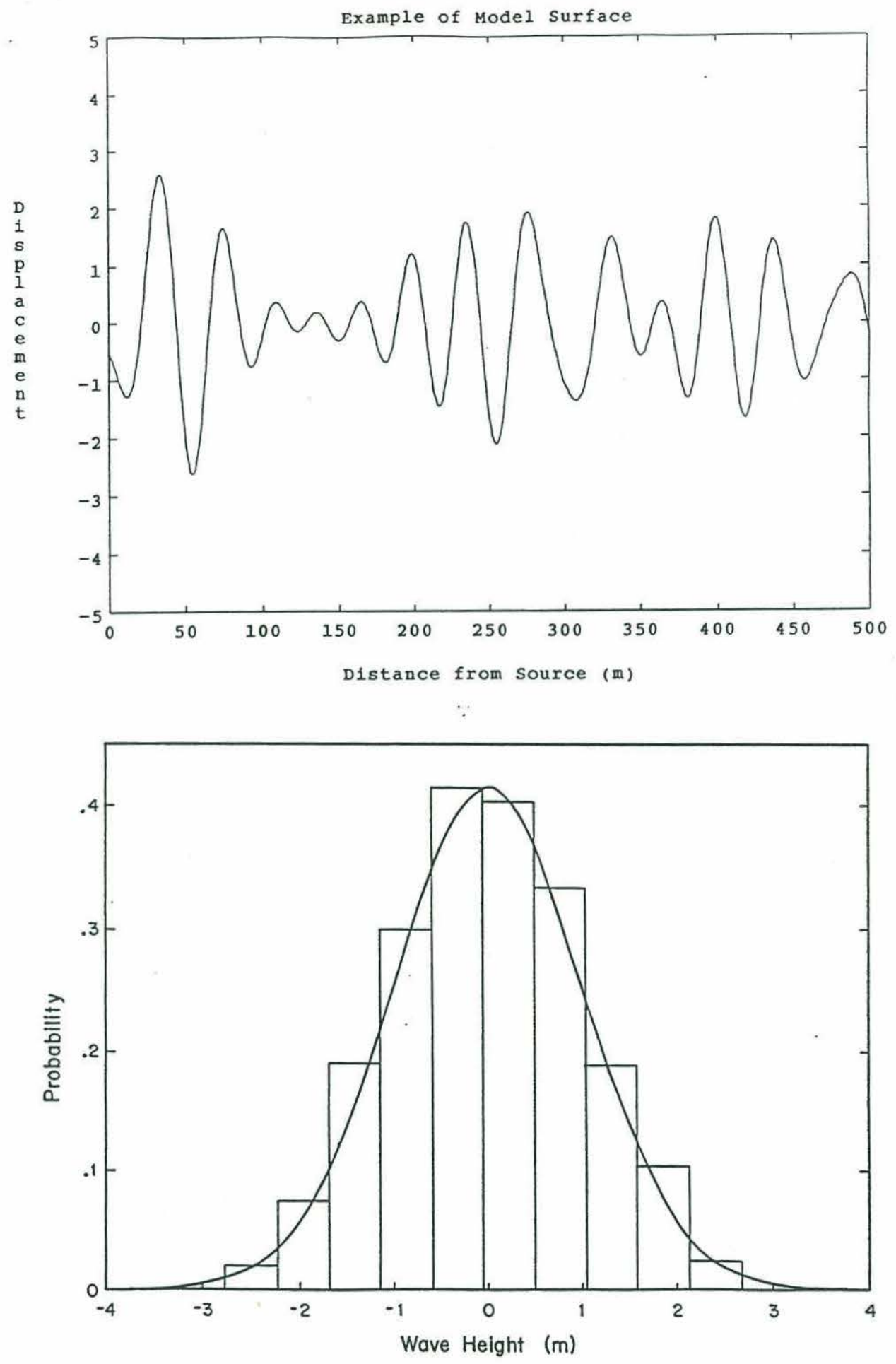

Figure 2-4: (a) A cross section of the model surface and (b) the corresponding surface height probability density function based on summing discete frequencies. 


\subsubsection{Surface Scattering Model}

The basic problem is to find the resultant pressure field at the receiver array given a source function which has been perturbated by interactions with a rough surface in a shallow water waveguide ${ }^{2}$. We start by analyzing the effects of a single reflection at a rough surface as illustrated in Figure 2-5.

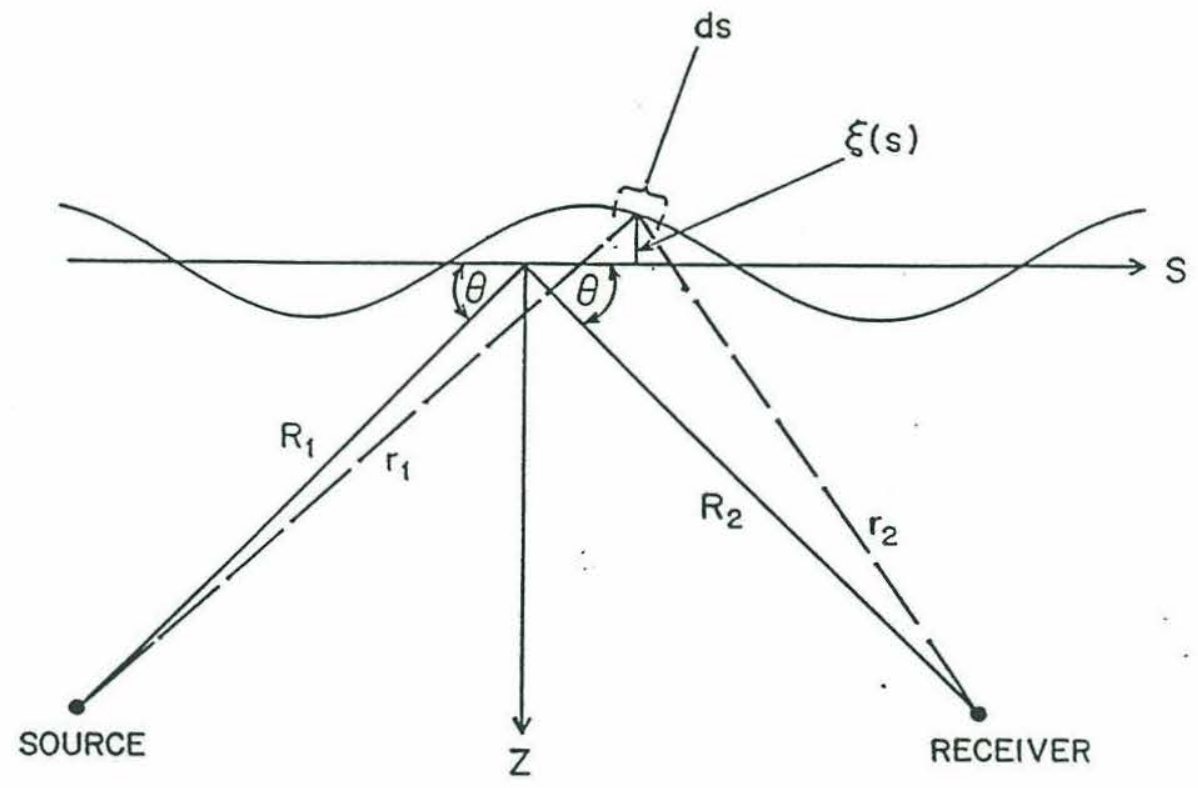

Figure 2-5: Idealized reflection from a rough sea surface in the specular direction.

In describing the scattering of the acoustic signal from the rough ocean surface, we follow a classic solution to the Helmholtz integral equation for acoustic waves on a random surface derived by Eckart (1953) using the Kirchhoff approximation for the boundary conditions. This provides the following result,

$$
P_{r}=\Re / 4 \pi \int_{S} P_{o} \frac{\partial}{\partial z}\left(\frac{e^{-i k\left(r_{1}+r_{2}\right)}}{r_{1}+r_{2}}\right) d s
$$

This solution was based on the following assumptions:

\footnotetext{
${ }^{2} \mathrm{~A}$ complete derivation is included in Appendix A.
} 
1. As stated by the Kirchhoff approximation, we assume that the reflection coefficient, which is derived using an infinite plane wave incident upon an infinite plane surface, can be applied at every point of the rough surface. There have been many studies on the accuracy of this approximation as well as its region of validity. A comprehensive study of scattering by McCammon (1984,p.131) concluded,

The Kirchhoff approximation is shown to agree rather well with the exact solution at very shallow grazing angles.

The validity of the approximation requires the radius of curvature of the sea surface to be much greater than the incident acoustic wavelength (Born and Wolf,1959). Specifically,

$$
\lambda \ll R_{c} \text { where } R_{c}=\frac{\left(1+\left(\frac{d \xi}{d s}\right)^{2}\right)^{3 / 2}}{\left|\frac{d^{2} \xi}{d s^{2}}\right|}
$$

The absolute lower bound for the curvature radius using our surface model is $\left[\sum_{n=1}^{5} a_{n} k_{n}^{2}\right]^{-1}$, making the stated region of validity

$$
\lambda \sum_{n=1}^{5} a_{n} k_{n}^{2} \ll 1
$$

The surface wavelengths, waveheights, and acoustic frequency used in our study are such that that the Kirchhoff approximation is within its accepted range of validity.

2. Following Eckart, we assume that the roughness of the surface is small enough so that the local normal to the surface $\left(\frac{\partial}{\partial n}\right)$ can be approximated by the normal to the mean surface $\left(\frac{\partial}{\partial z}\right)$. The region of validity of Eckart's approximation is generally accepted as

$$
\xi k \sin \theta \ll 1
$$

Given small sea surface heights (less than 2 meters rms), shallow grazing angles (less than $20^{\circ}$ ), and low frequency, our study meets the roughness criteria necessary for the small slope approximation.

3. We assume the effects of shadowing, multiple scattering, and curvature corrections can be neglected when calculating the specular reflection. As stated by McCammon (1984,p.112), 
... no corrections should be applied for the specular term to obtain the best possible fit to the exact solution, since all the examined corrections actually degrade[d] the solution at low grazing angles.

4. During the propagation of the signal from source to receiver, we assume the ocean surface is 'frozen'. This allows us to ignore frequency spreading due to interaction with a moving boundary and small changes in wave heights which would result from a moving wave. Considering the transit time of the acoustic signal versus the velocity of the surface waves, this is a reasonable assumption. The surface does 'move' between samples of the source signal in accordance with Equation (2.6).

Using a stationary phase approximation and assuming that $R_{1}$ and $R_{2}$ are much greater than the surface displacements, Equation(2.8) can be futher simplified to,

$$
P_{r}=P_{o} \frac{e^{-i k\left(R_{1}+R_{2}\right)}}{R_{1}+R_{2}} \underbrace{\Re \int_{S} e^{-i 2 \gamma_{1} \xi(s)} d s}
$$

This describes a ray undergoing reflection at a mean surface whose coherent reflection coefficient, $\langle\Re\rangle$, is calculated by integrating over the area of surface illumination. When the surface roughness correlation distance is much smaller than the illumination radius, the coherent reflection coefficient in the specular direction for a random, Gaussian distributed surface is $\langle\Re\rangle=\Re e^{-2 \gamma_{1}^{2} \sigma^{2}}$, where $\sigma^{2}$ is the variance of the sea surface displacement $\xi(s)$.

In order to test our scattering results, we numerically calculate the coherent reflection coefficient using Equation (2.12) for grazing angles of 0 to 18 degrees, assuming a large illumination area. This is compared to the theoretical coherent relection coefficient calculated for a Gaussian rough surface as shown in Figure 2-6. The solid line is the result from the numerical calculation over our rough surface. The dashed line is the theoretical result. The variance of sea surface displacement was fixed at 1.0 meter and $\Re$ assumed to be 1 . Even though our model consists of only a few fequencies of the total wave spectrum, the agreement to the full rough surface result is excellent, lending support to our use of a surface model with only a few degrees of freedom. 


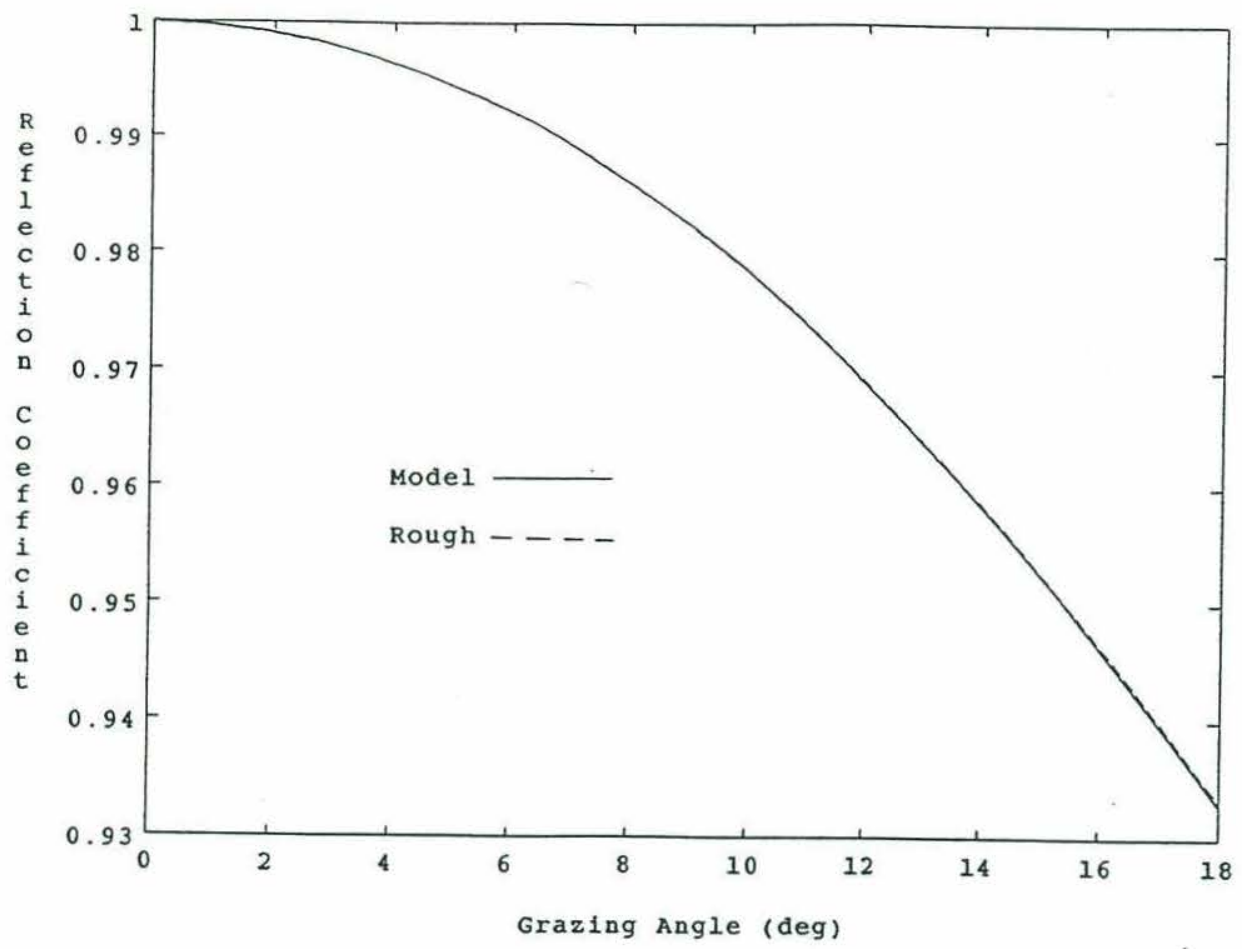

Figure 2-6: Comparison of the reflection coefficient numerically calculated for the model surface vs. the theoretical Gaussian rough surface.

Returning to the original problem of calculating the pressure field across an array in a shallow water waveguide, we now incorporate a new complex random variable, $B_{l m}(\mathbf{r})$, which is the effective rough surface reflection coefficient for the $l^{\text {th }}$ eigenray path to the $m^{\text {th }}$ sensor. This new variable, which is a function of the surface roughness, ray grazing angle, and surface illumination area, is defined as

$$
B_{l m}(\mathrm{r})=\prod_{n=0}^{n_{l}}\left[\int_{S} e^{-i 2 \gamma_{1} \xi_{n}(s)} d s\right]
$$

where $n_{l}$ is the total number of surface reflections for the $l^{\text {th }}$ eigenray. The total acoustic field along the array, after the addition of this variable, is written as

$$
P\left(\mathbf{z}_{m}, \omega \mid \mathbf{r}\right)=\sum_{l=1}^{L} A_{l m}(\mathbf{r}) B_{l m}(\mathbf{r}) e^{j \omega T_{l m}(\mathbf{r})}
$$

As a final validation of the surface wave model and our scattering assumptions, we recalculate the transmission loss curve in our Pekeris waveguide using Equation (2.14) 
and compare it to an 'exact' solution. The 'exact' transmission loss curve is calculated by SAFARI using a rough surface with zero correlation distance. Both calculations used an rms wave height of 1 meter. The results, displayed in Figure 2-7, show an even better agreement than the flat surface case. The rough surface has resulted in a simplified acoustic field which the ray calculations can more easily approximate.

\section{TRANSMISSION LOSS}

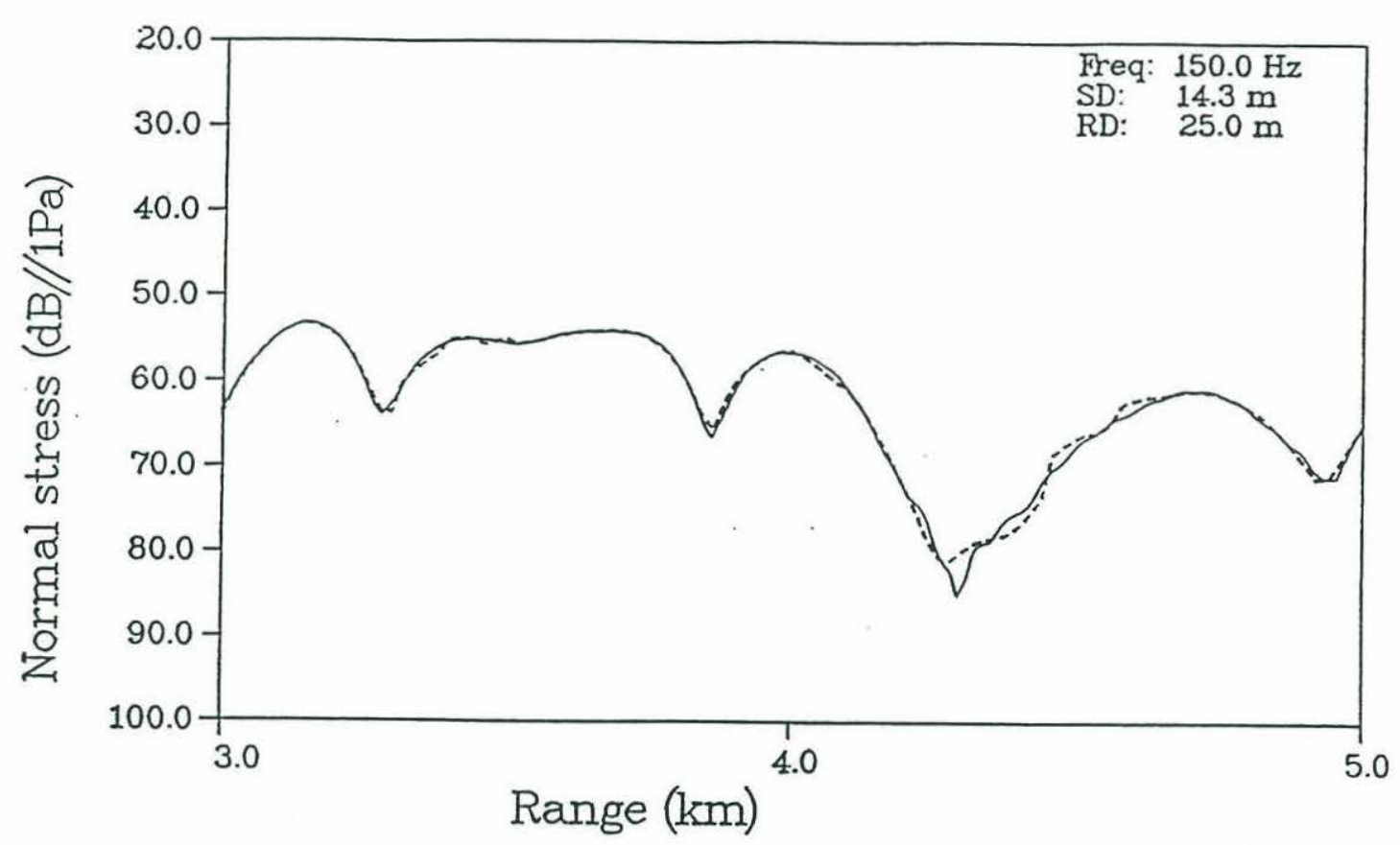

Figure 2-7: Comparison of transmission loss curves based on ray theory with beam displacement (dashed line) and the actual solution (solid line) including the effect of a random rough surface with $1 \mathrm{~m}$ rms wave height.

\subsection{Internal Wave Model}

In our internal wave model, which is based on CTD measurements of the surface mixed layer made off San Francisco (Lynch, pers comm), we assume a two layer shallow water channel (warm surface layer and cooler sublayer) overlying a sediment halfspace bottom. The two layers water layers form a discontinuity at which the energy of the internal waves is concentrated. Using the linearized hydrodynamic equations, it is easily 
shown the resulting wave perturbations have solutions of the form:

$$
\zeta(s, t)=\sum_{n=1}^{\infty} a_{n} \cos \left(k_{n} s+\omega_{n} t+\epsilon_{n}\right)
$$

The dispersion relationship for shallow water ${ }^{3}$ internal waves is shown to be (Apel, 1987),

$$
\omega_{n}^{2}=k_{n}^{2} g\left(\frac{\rho_{1}^{\prime}-\rho_{1}}{\rho_{1}}\right)\left(\frac{h_{1}^{\prime} h_{1}}{h_{1}^{\prime}+h_{1}}\right)
$$

where $\rho_{1}^{\prime}$ is the density in the warmer surface layer of depth $h_{1}^{\prime}$ and $\rho_{1}$ is the density in the rest of the water column of depth $h_{1}$. In general, shallow water internal waves are characterized by amplitudes of a few meters and wavelengths from tens of meters to kilometers. The propagation velocities of internal waves fall in the range between a few tens of centimeters per second to several meters per second but are generally much slower than surface waves. An excellent example of an internal wave field was recorded using high frequency acoustic scattering by Orr (1980) and is shown in Figure 2-8. As seen, there is almost a sinusoidal interface between a warmer surface layer and the underlying cooler water (arrows 1 and 4). Scattering at 35 meters (arrows 2 and 3 ) result from stratified point scatterers. Heavy scattering near 75 meters is probably caused by marine life.

Using a ray tracing scheme, the acoustic pressure field in the presence of an single frequency internal wave was first calculated by Lee (1961), who showed that the sound field will be alternately of high and low intensity corresponding to the wavelength of the sinusoidal internal wave. The intensity fluctuation results from the refraction of the ray paths which tend to periodically group and disperse. This is unlike surface scattering whose intensity structure results from interference of rays that have been shifted in phase at the rough interface. Katz (1966) illustrated the fluctuation of the intensity field with time as the internal wave propagated along the discontinuity. An example of a single frequency internal wave model is shown in Figure 2-9 together with a typical ray path. An expanded view of the ray path crossing the internal wave displays the fluctuations of the intensity field. The intensity field was calculated by a raytracing procedure.

\footnotetext{
${ }^{3}$ The total depth in our model is great enough so that only very long internal waves would satisfy the shallow water assumption. We have actually used the complete dispersion relationship found in Apel to compute the propagation velocity. The difference is found only to be $10 \%$ so that Equation (2.16) could simply be used as a good approximation.
} 


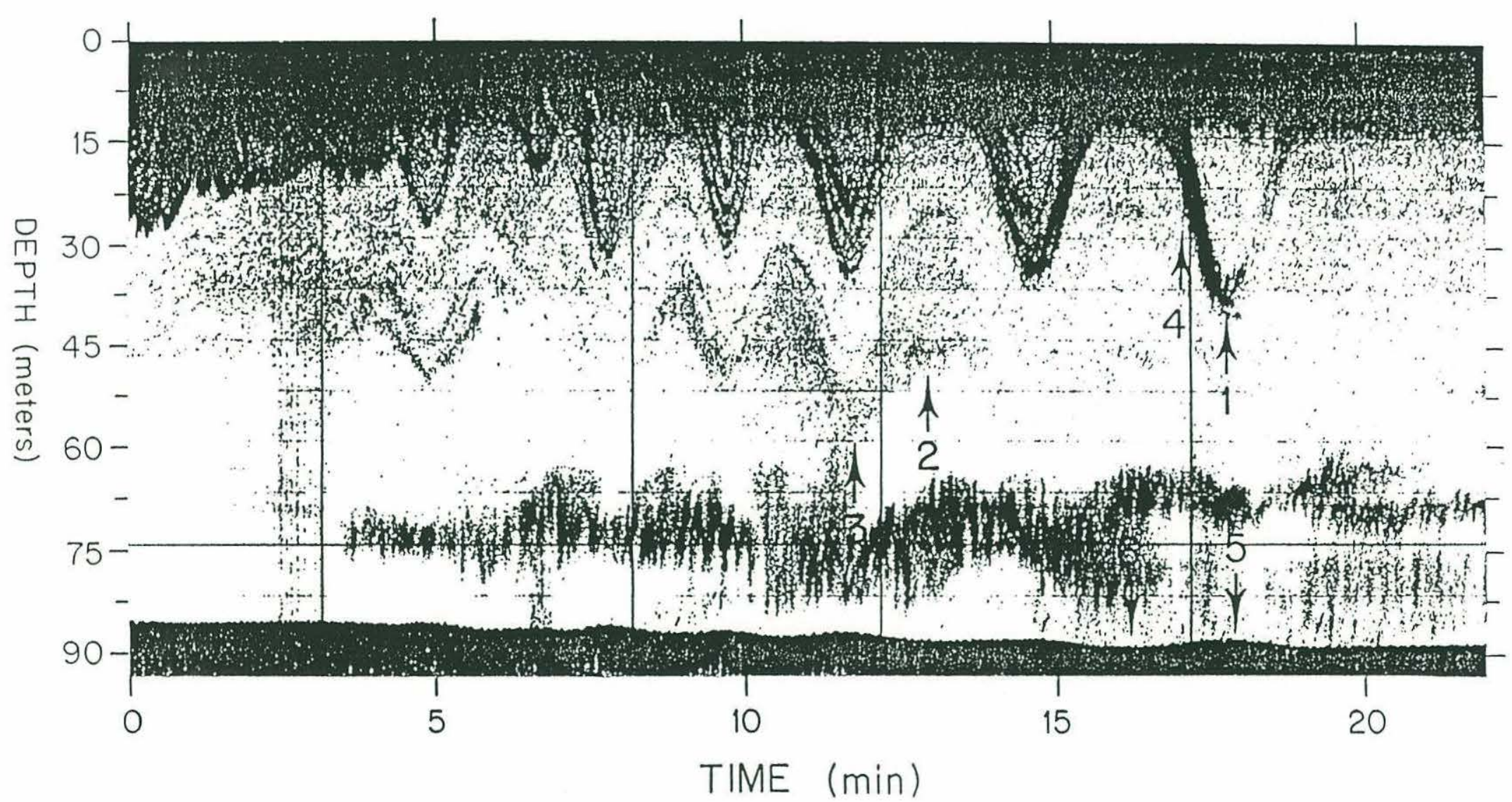

Figure 2-8: An acoustic record of an internal wave field. The period of the waves are doppler shifted by the ship's speed of about 2.5 knots. 
AIR
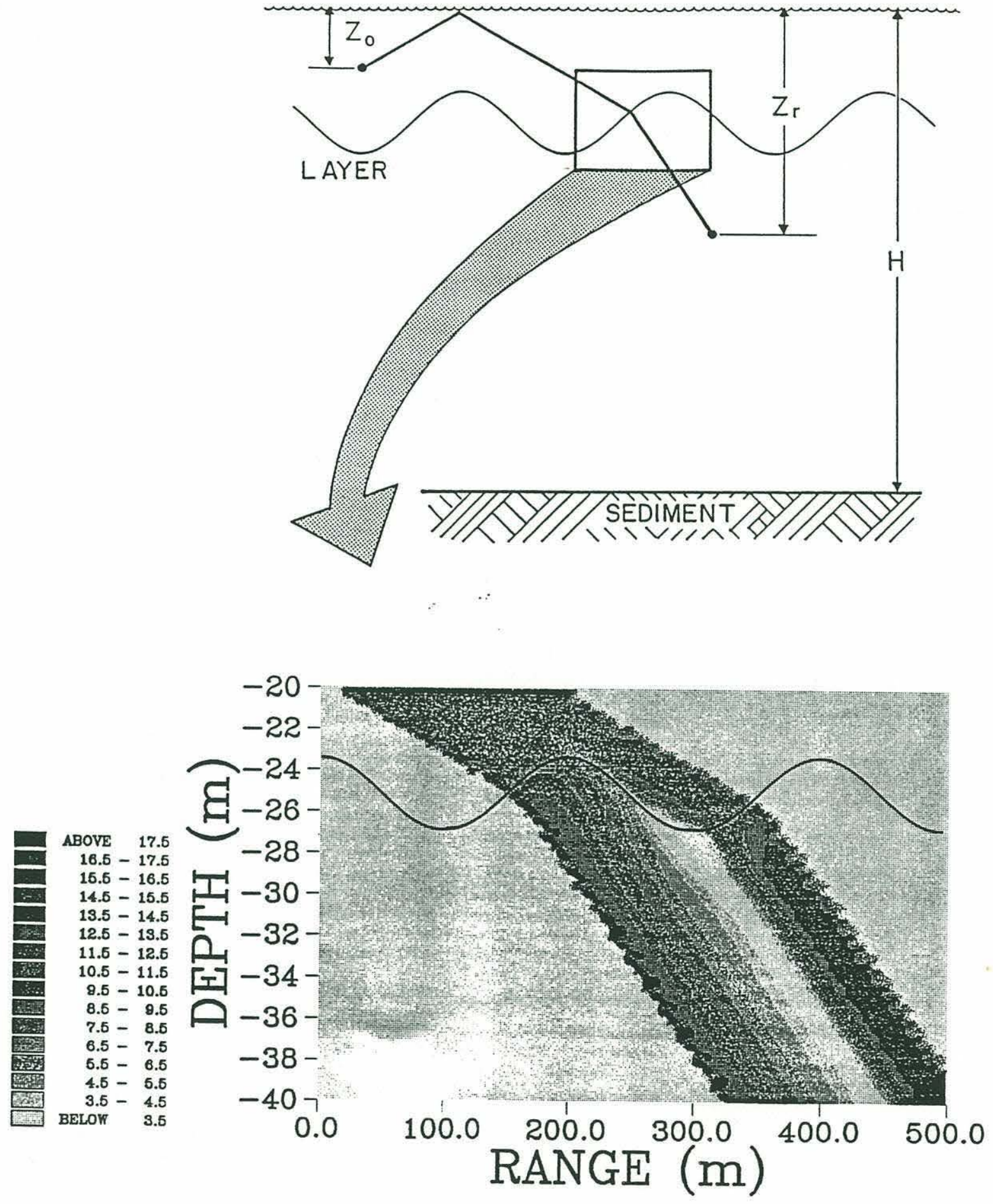

Figure 2-9: (a) A typical ray path through a shallow water internal wave (b) An expanded view showing the intensity fluctuations of the ray. 
Although the pressure field at each array element in our model could be calculated by an iterative raytracing procedure, this would require extensive calculations for each source/receiver pair. Given that matched field processing requires very large numbers of source/receiver pairs, it would be infeasible to utilize this raytracing technique. Instead, we shall utilize a technique well known in surface scattering - the idea of a phase screen, or in this case an intensity screen. Basically, the concept is one of projecting the intensity perturbation of the internal wave on a flat plane located at the mean interface height. Since the effect of the internal wave is not simply described analytically, we numerically calculate the intensity perturbation of the sound field using the raytracing procedure. Instead of recalculating the intensity correction each time the ray passes through the internal wave, we define a table lookup as a function of the incident angle and internal wave phase. This correction is then applied to the ray at the mean interface of the internal wave. By this method, one can analytically solve for the path of the eigenray using the three layer model and apply the internal wave correction at the point where the raypath intercepts the intensity screen.

We make the following assumptions in our calculations,

1. Modified ray theory is still an adequate approximation to the acoustic field for the three layer model. As a check, we once again recalculated the transmission loss curves for our three layer model using modified ray theory and SAFARI. The results, displayed in Figure 2-10 show a fairly good agreement between the exact solution and modified ray theory.

2. The radius of curvature of the internal wave interface is large relative to the acoustic wavelength so that geometric optics can be applied with negligible scattering. Based on a single frequency sinusoidal internal wave on the order of 200 meters wavelength and 2 meters amplitude, the region of validity requires,

$$
\lambda a k^{2} \approx .02 \ll 1
$$

where $\lambda$ is the acoustic wavelength, $a$ is the internal wave amplitude, and $k$ is the internal wave spatial wavenumber. 


\section{TRANSMISSION LOSS}

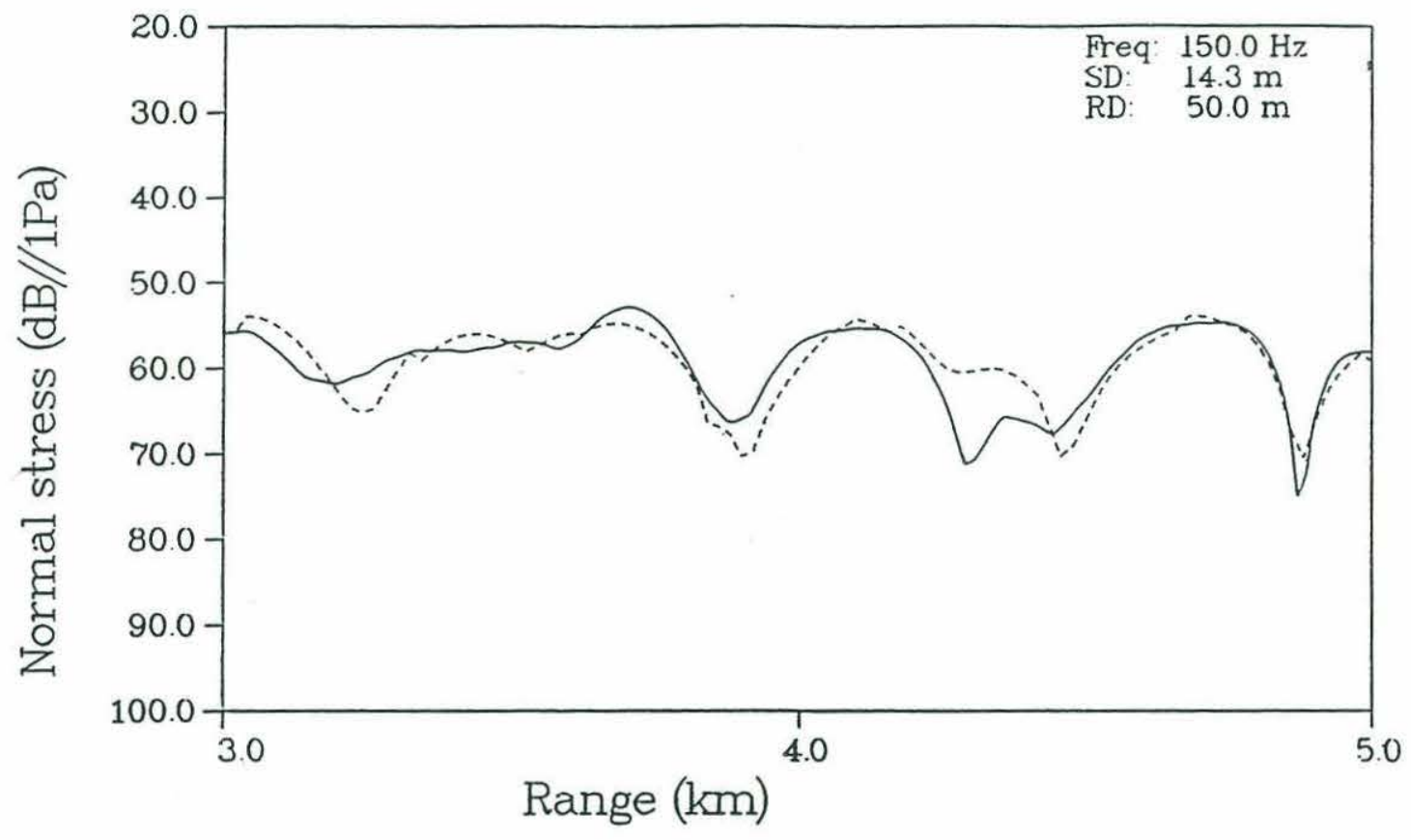

Figure 2-10: Comparison of transmission loss curves based on ray theory with beam displacement (dashed line) and the actual solution (solid line) for the three layer internal wave model. 
3. The sound speed differential between the two layers is small such that the phase differential between a ray which is incident at the mean interface and a ray which is incident at the actual interface of the internal wave can be ignored, i.e.,

$$
a\left(\gamma_{1}^{\prime}-\gamma_{1}\right) \ll \pi / 4
$$

where $\gamma_{1}^{\prime}$ and $\gamma_{1}$ are the vertical wavenumbers in the warmer and cooler water layers, respectively and $a$ is the amplitude of the internal wave. Given that $\delta c=5 \mathrm{~m} / \mathrm{sec}$ and low grazing angles (5-15 degrees), the phase differential is approximately $\pi / 150$ which is small enough to be ignored.

4. The density and sound speed differentials between the two water layers are large enough to cause internal wave perturbations, yet small enough so that total transmission of the signal occurs with no significant reflective component. Based on a plane wave incident from the upper layer (where the source is located) upon the lower cooler layer, the reflection coefficient is

$$
|\Re|=\frac{\left|\frac{\rho_{1}^{\prime} c_{1}^{\prime}}{\sin \theta_{1}^{\prime}}-\frac{\rho_{1} c_{1}}{\sin \theta_{1}}\right|}{\left|\frac{\rho_{1}^{\prime} c_{1}^{\prime}}{\sin \theta_{1}^{\prime}}+\frac{\rho_{1} c_{1}}{\sin \theta_{1}}\right|}
$$

At normal incidence, $|\Re| \approx .0005$. But at low grazing angles (below 5 degrees), $|\Re| \geq$ .1 which would seem to violate the assumption. However, at low grazing angles, the ray also undergoes significant intensity loss due to spreading which dominates the calculation. If we look instead at the total intensity loss across the layer (defined as Transmission Coefficient $\times$ Spreading Loss), we get a better estimate of the accuracy of our approximation. In Figure 2-11, we have plotted the total intensity loss as a function of grazing angle using the approximation that the transmission coefficient is always equal to one (dashed line) and using the actual transmission coefficient calculated in Equation (2.19) (solid line). We observe that the the total intensity loss is very similar for both cases and supports our use of the total transmission approximation.

Once again, we return to the original problem of calculating the pressure field across an array in a shallow water waveguide. We incorporate a third random variable, $C_{l m}(\mathbf{r})$, 


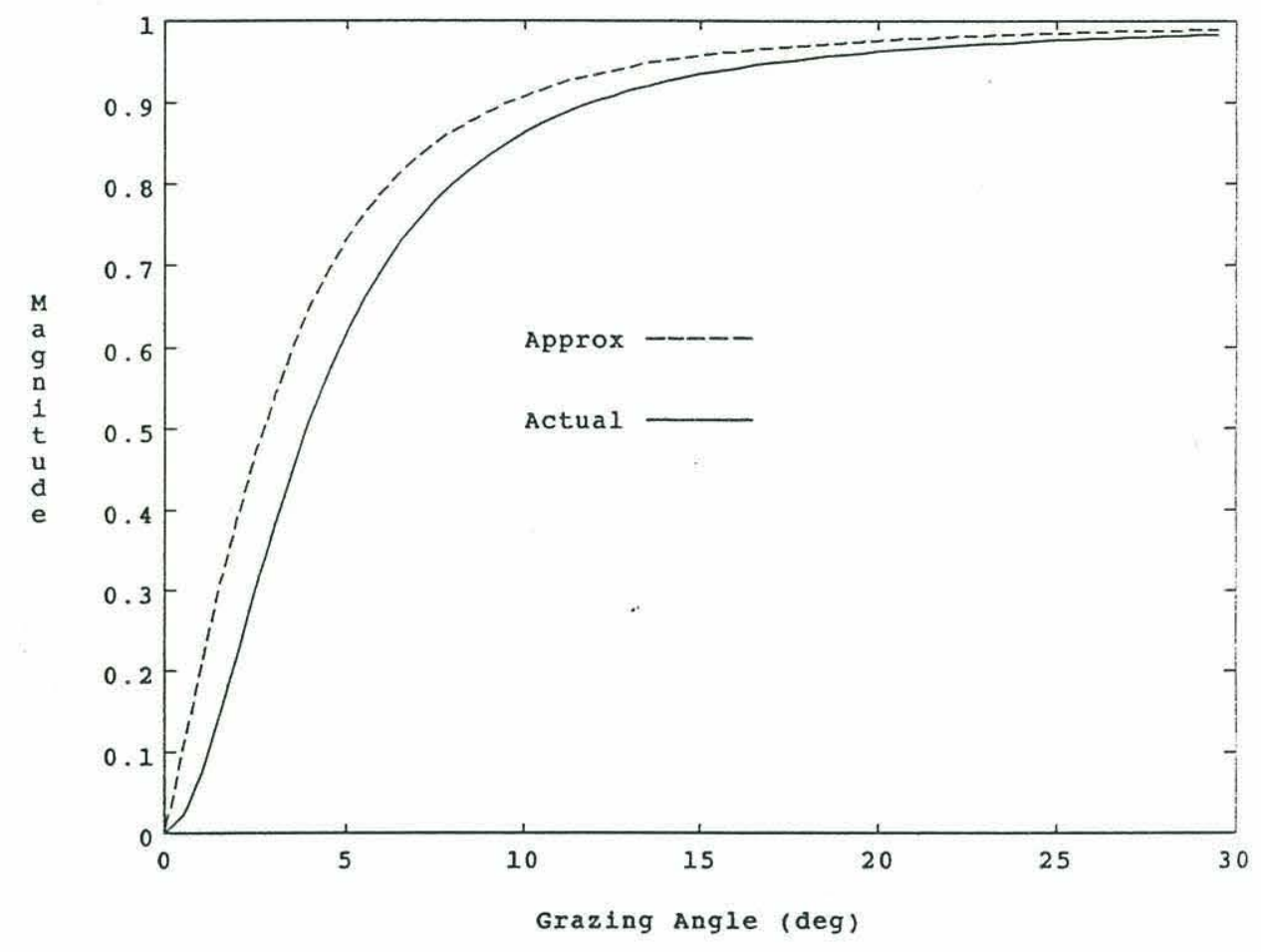

Figure 2-11: Total intensity loss across the layer using an approximation of total transmission (dashed line) and the actual transmission coefficient (solid line). Both include spreading losses. 
which is the effective intensity change due to internal wave refraction for the $l^{\text {th }}$ eigenray path to the $m^{\text {th }}$ sensor from a source located a $\mathbf{r}$. The total acoustic field along the array, after the addition of this variable, is written as

$$
P\left(\mathbf{z}_{m}, \omega \mid \mathbf{r}\right)=\sum_{l=1}^{L} A_{l m}(\mathbf{r}) B_{l m}(\mathbf{r}) C_{l m}(\mathbf{r}) e^{j \omega T_{l m}(\mathbf{r})}
$$

\subsection{Matched Field Processing}

The generalized matched field beamformer correlates the measured field at the array with replicas of the expected field from all postulated source positions. The measured field is composed of the signal field, $P\left(\mathbf{z}_{m}, \omega \mid \mathbf{r}\right)$ and noise $n\left(\mathbf{z}_{m}, \omega\right)$, i.e.,

$$
S\left(\mathbf{z}_{m}, \omega \mid \mathbf{r}\right)=P\left(\mathbf{z}_{m}, \omega \mid \mathbf{r}\right)+n\left(\mathbf{z}_{m}, \omega\right)
$$

For simplicity, we will assume the noise to be uncorrelated, Gaussian, and white with a spectral level of $\sigma_{n}^{2}$. In doing so, we neglect the effect of distributed noise sources, such as surface or shipping noise, as well as discrete sources other than the original. Correlated noise effects have recently been treated by Baggeroer et. al. (1988).

\subsubsection{Bartlett Beamformer}

Bartlett (BE), or conventional, matched field beamforming is performed by weighting the output of the array elements and summing over all the elements, i.e.

$$
L_{B E}(\hat{\mathbf{r}})=\left|\sum_{m=1}^{M} w_{m}(\hat{\mathbf{r}}) S_{m}(\mathbf{r})\right|^{2}=\left|\mathbf{w}_{B E}^{\dagger}(\hat{\mathbf{r}}) \mathrm{S}(\mathbf{r})\right|^{2}
$$

where

- the $\dagger$ sign superscript denotes the conjugate transpose,

- $M$ is the number of array elements,

- $\mathbf{r}$ is the actual source location in depth and range,

- $\mathbf{S}(\mathbf{r})$ is the signal field generated by a source located at $\mathbf{r}$,

- $\hat{\mathbf{r}}$ is the postulated source location(s), and 
- $\mathbf{w}_{B E}(\hat{\mathbf{r}})$ is the weighting vector of the BE processor for the look direction $\hat{\mathbf{r}}$.

The estimator forms a complicated pattern, known as the ambiguity surface, which is a function of the scanning parameters of depth and range. A large value of the estimator would indicate a likely source position. However, due to the complex nature of the channel, there may well be several locations where the estimator reaches a high value. Yet only one is the true source location whereas the others form a set of ambiguous peaks.

For a Bartlett beamformer, we set the weighting vectors equal to the replica fields $\Psi(\hat{\mathbf{r}})$, which are the normalized acoustic fields across the array based on predicted source locations. We adopt the normalization that $\Psi^{\dagger}(\hat{\mathbf{r}}) \Psi(\hat{\mathbf{r}})=1$. Therefore, given that

$$
\mathbf{w}_{B E}(\hat{\mathbf{r}})=\Psi(\hat{\mathbf{r}}),
$$

the output of the Bartlett beamformer can be written,

$$
L_{B E}(\hat{\mathbf{r}})=\Psi^{\dagger}(\hat{\mathbf{r}}) \mathbf{K}(\mathbf{r}) \Psi(\hat{\mathbf{r}})
$$

where $\mathbf{K}(\mathbf{r})$ is the total covariance matrix for the array. Using the white noise assumption, we can write the covariance matrix as

$$
\mathbf{K}(\mathbf{r})=\mathbf{S}(\mathbf{r}) \cdot \mathbf{S}^{\dagger}(\mathbf{r})=\sigma_{n}^{2} I+\sigma_{s}^{2} \Phi(\mathbf{r}) \Phi^{\dagger}(\mathbf{r})
$$

where $\sigma_{s} \Phi(\mathbf{r})$ has been substituted for the signal field, $P\left(\mathbf{z}_{m}, \omega \mid \mathbf{r}\right) . \Phi(\mathbf{r})$ is the normalized signal field such that $\operatorname{tr}\left(\Phi(\mathbf{r}) \Phi^{\dagger}(\mathbf{r})\right)=M$ (the number of array elements) and $\sigma_{s}$ is the average signal strength across the array. Substitution of Equation (2.25) into Equation (2.24) yields a more direct form of the Bartlett processor,

$$
L_{B E}(\hat{\mathbf{r}})=\sigma_{n}^{2}+M \sigma_{s}^{2} \underbrace{\left.\frac{\Psi^{\dagger}(\hat{\mathbf{r}}) \Phi(\mathbf{r})}{M}\right|^{2}}_{\rho^{2}(\hat{\mathbf{r}}, \mathbf{r})}
$$

The variable $\rho^{2}$ is the correlation coefficient between the measured field and the replica fields. In essence, it is a measure of the similarity between the source field at $\mathbf{r}$ and the replica field(s) at $\hat{\mathbf{r}}$. The normalization of the replica fields results in values for $\rho^{2}$ varying from 0 , when there is no correlation, to 1 when there is an exact correlation. An analysis of the estimator given in Equation (2.26) shows that the first term is a constant which 
contributes a smooth background noise floor on the ambiguity surface. All local maxima on the ambiguity surface result from the second term which is dependent only on the source and replica fields.

\subsubsection{Maximum Likelihood Beamformer}

More recently, studies of matched field processing have centered upon the use of the Maximum Likelihood Method (MLM) for the parameter estimation procedure as developed by Capon (1969) and Hinich (1973). Basically, the algorithm adaptively constructs a weight vector $\left(\mathbf{w}_{M L M}\right)$ which yields a minimum mean-square output to the noise field with the constraint that the signal field is passed with unity gain when $\hat{\mathbf{r}}=\mathbf{r}$. In other words, the weight vector minimizes the quadratic function,

$$
F=\mathbf{w}_{M L M}^{\dagger}(\hat{\mathbf{r}}) \mathbf{K}(\mathbf{r}) \mathbf{w}_{M L M}(\hat{\mathbf{r}})+\lambda\left(\mathbf{w}_{M L M}^{\dagger}(\hat{\mathbf{r}}) \Psi(\hat{\mathbf{r}})-1\right)
$$

where $\lambda$ is a Lagrange multiplier. Using the calculus of variations, the optimal weight vector is derived to be

$$
\mathrm{w}_{M L M}(\hat{\mathbf{r}})=\frac{\mathrm{K}^{-1} \Psi(\hat{\mathbf{r}})}{\Psi^{\dagger}(\hat{\mathbf{r}}) \mathrm{K}^{-1} \Psi(\hat{\mathbf{r}})}
$$

Substituting $\mathrm{w}_{M L M}$ in Equation (2.28) for $\mathrm{w}_{B E}$ in Equation (2.22), we can define the output of the processor by

$$
L_{M L M}(\hat{\mathbf{r}})=\left[\Psi^{\dagger}(\hat{\mathbf{r}}) \mathrm{K}^{-1}(\mathbf{r}) \Psi(\hat{\mathbf{r}})\right]^{-1}
$$

By using the Woodbury identity for matrix inversion which states

$$
\left[\mathrm{A}+\mathrm{BC}^{\dagger}\right]^{-1}=\mathrm{A}^{-1}-\mathrm{A}^{-1} \mathrm{~B}\left(\mathrm{I}+\mathrm{C}^{\dagger} \mathrm{A}^{-1} \mathrm{~B}\right) \mathrm{C}^{\dagger} \mathrm{A}^{-1}
$$

where $\mathrm{A}$ is an $M \times M$ matrix, $\mathrm{B}$ and $\mathrm{C}$ are $M \times N$ matricies, and $\mathrm{I}$ is the identity matrix of rank $N$, the MLM estimator can be rewritten

$$
L_{M L M}(\hat{\mathbf{r}})=\frac{\sigma_{n}^{2}+M \sigma_{s}^{2}}{1+M \sigma_{s}^{2} / \sigma_{n}^{2}\left[1-\rho^{2}\right]}
$$

We see that if there is no correlation between the measured field and the replica, the estimator reduces approximately to the noise level. If there is a high correlation, the estimator equals the signal plus noise, as desired. The $\left[1-\rho^{2}\right]$ term, referred to as 
the 'estimator-subtractor' by Van Trees (1968), is responsible for acheiving the desired sidelobe minimization.

To fully understand matched field processing in a shallow water waveguide, it is instructive to first examine the characteristics of the processor for the case of plane waves in an infinite medium. A comparison between the performance of a $\mathrm{BE}$ processor and an MLM processor is illustrated in Figures 2-12(a) and (b). A vertical array with 21 elements spaced 2.5 meters apart is placed in an infinite fluid medium with sound speed of 1500 $\mathrm{m} / \mathrm{s}$ and density of $1 \mathrm{gm} / \mathrm{cc}$. A $150 \mathrm{~Hz}$ plane wave is incident on the array at a angle of zero degrees. The plane wave ambiguity functions have been plotted for various signalto-noise ratios (SNR). In general, the Bartlett beamformer has significant sidelobes which can often be indistinguishable from the mainlobe. The MLM matched field processor has better suppression of sidelobes and higher resolution of the mainlobe.

The enhanced performance is particularly significant when the signal-to-noise ratio is high. For plane wave beamforming, this is usually considered advantageous. However, for MLM processing in a waveguide the high resolution can result in severe degradation of the estimator. First, the grid spacing of the scanning parameters must be decreased to compensate for the more narrow peak. This in turn requires extensive numerical calculations to produce the replica fields. Even then, an 'infinite' number can not be determined and some mismatch can still occur. Secondly, the MLM processor is sensitive not only to errors in the scanning parameters but also errors in the model itself. In this case, even if the scanning parameters are sampled exactly at the true values, the replica field may not provide a perfect match to the measured field. A more useful processing method would adequately control sidelobes, like the MLM processor, yet be more tolerant of slight mismatches between the measured field and the predicted field, like the $\mathrm{BE}$ processor. The next two sections present alternative algorithms which attempt to satisfy this criteria.

\subsubsection{Maximum Constraint Beamformer}

Baggeroer and Schmidt (pers. comm.) are developing a technique which uses a Multiple Constaint Method (MCM) for parameter estimation. Simply stated, the method 

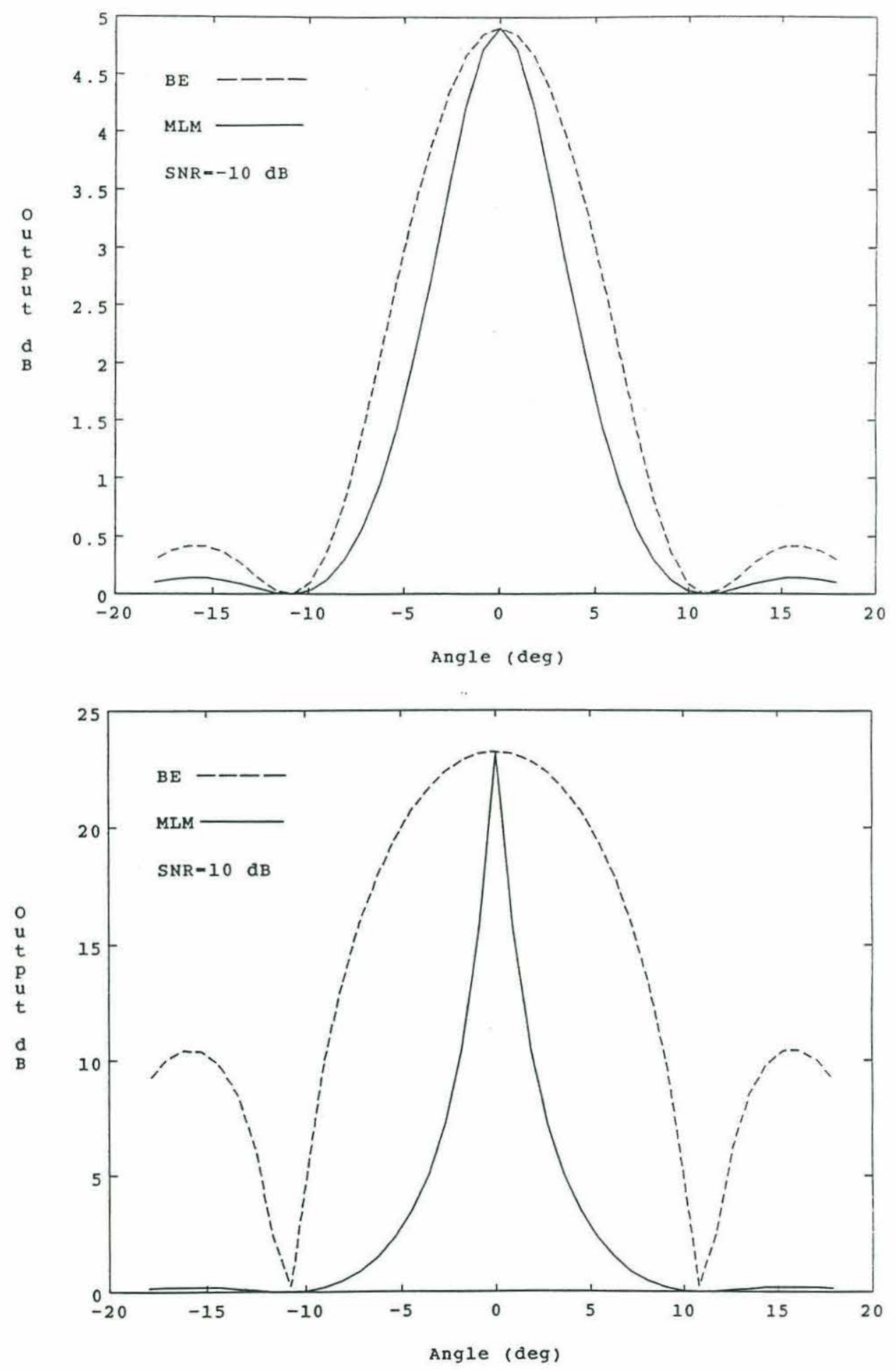

Figure 2-12: Plane wave ambiguity functions for a $B E$ processor(dashed line) and an MLM processor(solid line) given an SNR of (a)-10 dB and (b) $10 \mathrm{~dB}$. 
attempts to match the measured field not just to a single grid point, like the MLM processor, but to a small group of grid points which are close enough in depth and range that they have similar replica fields, but not exactly the same. We provide here a brief summary of the derivation of the MCM processor.

The quadratic form constraining the output of the MCM beamformer is minimized with the multiple constraints that the signal field is passed with unity gain throughout the expanded grid area:

$$
F=\mathbf{w}_{M C M}^{\dagger}(\hat{\mathbf{r}}) \mathrm{K}(\mathbf{r}) \mathbf{w}_{M C M}(\hat{\mathbf{r}})+\sum_{n=1}^{N} \operatorname{Real}\left[\lambda_{n}\left(\mathbf{w}_{M C M}^{\dagger}(\hat{\mathbf{r}}) \Psi\left(\hat{\mathbf{r}}_{n}\right)-d_{n}\right)\right]
$$

where $\mathrm{w}_{M C M}$ is the weighting vector desired by the MCM processor, $\Psi\left(\hat{\mathrm{r}}_{n}\right)$ is the replica field generated by a source located at the $n^{\text {th }}$ grid point contained in the enlarged grid area centered at $\hat{\mathbf{r}}$, the $\lambda_{n}$ are Lagrange multipliers, and the $d_{n}$ are constraints over the $N$ grid points defining the enlarged grid area. Using calculus of variations, the output of the MCM processor is shown to be

$$
L_{M C M}(\hat{\mathbf{r}})=\frac{\mathrm{D}\left[\bar{\Psi}^{\dagger}(\hat{\mathbf{r}}) \mathrm{K}^{-1}(\mathbf{r}) \bar{\Psi}(\hat{\mathbf{r}})\right]^{-1} \mathrm{D}^{\dagger}}{\mathrm{D}\left[\bar{\Psi}^{\dagger}(\hat{\mathbf{r}}) \bar{\Psi}(\hat{\mathbf{r}})\right]^{-1} \mathrm{D}^{\dagger}}
$$

where $\bar{\Psi}(\hat{\mathbf{r}})$ is a matrix composed of the $N$ grid point replicas forming the enlarged grid area, such that

$$
\bar{\Psi}(\hat{\mathbf{r}})=\left[\Psi_{1}|\cdots| \Psi_{\hat{\mathbf{r}}}|\cdots| \Psi_{\mathrm{N}}\right], \mathbf{D}=\left[d_{1}|\cdots| d_{\hat{\mathbf{r}}}|\cdots| d_{N}\right] \text {, and } d_{n}=\Psi_{\hat{\mathbf{r}}}^{\dagger} \Psi_{n} \text {. }
$$

The selection of grid locations is of particular importance for the MCM processor. If the grid points are too far apart, the replica fields are so unlike that significant sidelobes occur. If the grid points are too close together, significant numerical errors could occur due to the singularity of the replica matrix. In addition, a small spacing results in a significant increase in computational requirements. For a plane wave example which we will discuss next, we have selected grid points approximately 1 degree apart and chosen the expanded grid area to include the look direction grid point and its nearest neighbor on each side.

The robustness of the MCM beamformer is illustrated for the same 21 element linear array described in the last section. A $150 \mathrm{~Hz}$ source is located in the far field such 
that the incident wave is modeled as a plane wave. The incident angle is 0 degrees and the average SNR at the receiver array is $10 \mathrm{~dB}$. The replica fields are also modeled as plane waves. In Figure 2-13(a), the ambiguity function for a BE processor(dashed line), an MLM processor(solid line), and an MCM processor(dash-dotted line) are plotted. One immediately notes that the MCM beamformer has a wide mainlobe, like the BE beamformer, while maintaining the desired sidelobe suppression of the MLM beamformer.

To illustrate the robustness of the MCM processor, the source is now located closer to the array (range $=1000$ meters) such that the incident wave has a curvature which prevents a perfect match with any plane wave replicas. The incident angle remains 0 degrees and the signal strength is adjusted such that the average SNR at the receiver is still $10 \mathrm{~dB}$. As shown in Figure 2-13(b), the MLM beamformer is highly susceptible to mismatch and therefore the output is greatly reduced. The MCM beamformer, however, appears unaffected.

\subsubsection{Variable Coefficient Likelihood Beamformer}

We introduce here a second processor, which we refer to as the Variable Coefficient Likelihood Method (VCLM). It has similar characteristics to the MLM processor, yet is even more robust to environmental mismatch. Basically, we define the VCLM processor as,

$$
L_{V C L M}(\hat{\mathbf{r}})=\left[\Psi^{\dagger}(\hat{\mathbf{r}}) \mathrm{K}^{-\alpha}(\mathbf{r}) \Psi(\hat{\mathbf{r}})\right]^{-1 / \alpha}
$$

where $\alpha$ is now a variable coefficient. For the case of white noise and a single source, the VCLM estimator can be rewritten,

$$
L_{V C L M}(\hat{\mathbf{r}})=\frac{\sigma_{n}^{2}+M \sigma_{s}^{2}}{\left[\rho^{2}+\left(1+M \sigma_{s}^{2} / \sigma_{n}^{2}\right)^{\alpha}\left(1-\rho^{2}\right)\right]^{1 / \alpha}}
$$

This is similar in appearance to a low pass filter where the signal-to-noise ratio and the exponent, $\alpha$, determine the cutoff point. When the correlation coefficient is equal to 1 , the output of the estimator is equal the the signal plus noise. When the correlation coefficient is less than 1 but greater than the cutoff point, the estimator is still approximately equal to the signal plus noise value. Below the cutoff point, the estimator performs much 

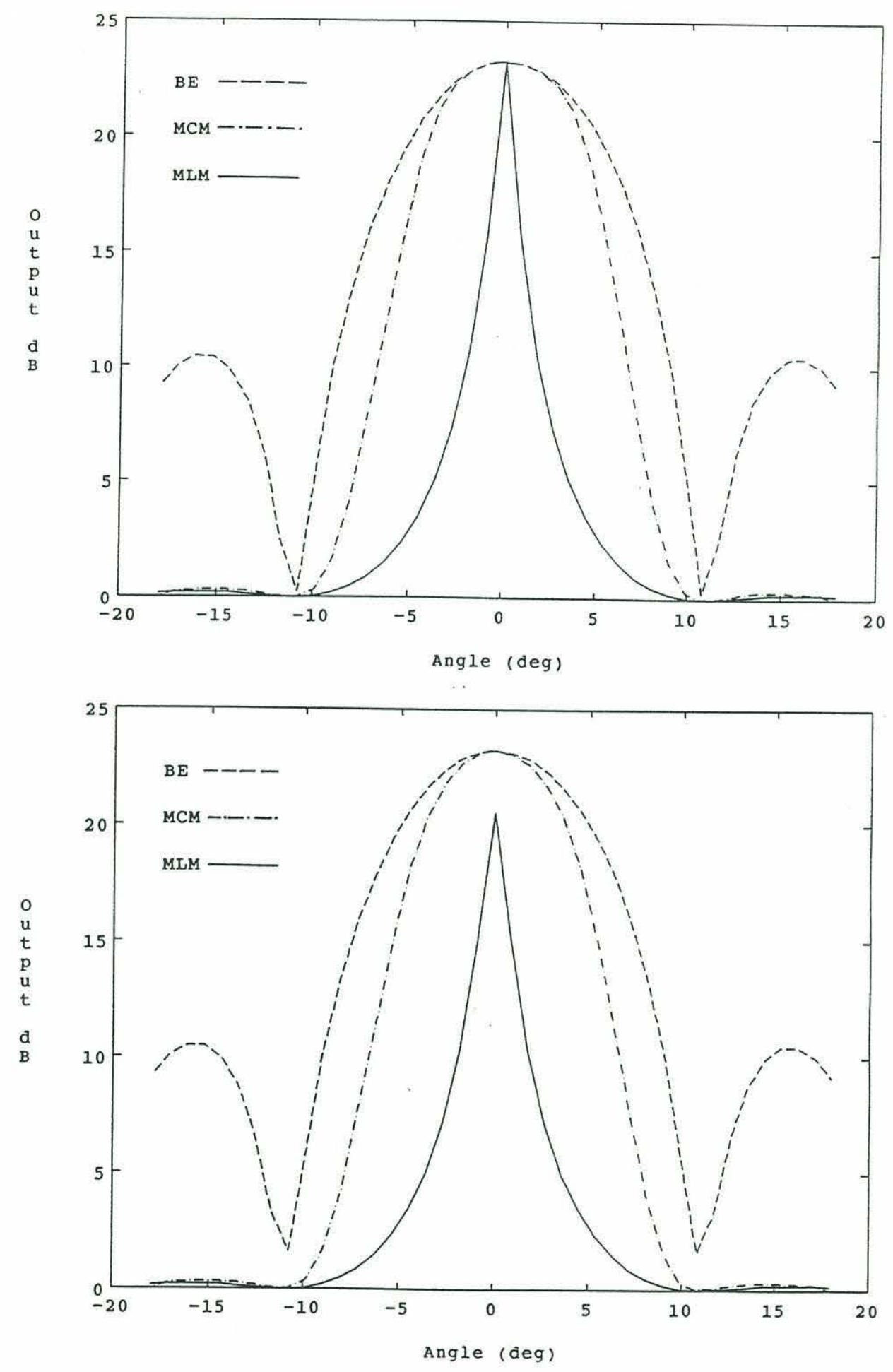

Figure 2-13: Plane wave ambiguity function for a $\mathrm{BE}$ processor(dashed line), an MLM processor(solid line), and an MCM processor(dash-dotted line) in the case of (a) no mismatch and (b) a mismatch caused by plane wave assumption with source close to array. 
like a normal MLM estimator except that the 'estimator-subtractor' term has a variable exponent to control the extent of sidelobe suppression.

Figure 2-14(a) shows the output of the VCLM processor vs. correlation coefficient for various values of the exponent. When the coefficient is equal to one the VCLM processor is exactly an MLM processor. As the exponent increases the output level is increased relative to the MLM output when the correlation coefficient is between 0 and 1. It appears that all we have accomplished is to raise the level of sidelobes. In a way that is true. But by looking at the difference between the output of the VCLM and the MLM beamformer in Figure 2-14(b), one can see that the sidelobe levels are increased by different amounts. Furthermore, by varying the exponent value the location of the difference peak can be shifted. Thus, by judicious selection of the exponent value, we can maximize the output of the source peak relative to all other ambiguous peaks.

The selection of the optimal exponent value is acomplished by finding the value of $\alpha$ which maximizes of the difference function $L_{V C L M}\left(\alpha, \mathrm{SNR}, \rho_{\max }^{2}\right)-L_{M L M}\left(\mathrm{SNR}, \rho_{\max }^{2}\right)$. This requires an estimate of the SNR and the maximum correlation coefficient between the source and the replica fields. We accomplish this by using a singular value decomposition of the covariance matrix (Dongarra, et.al.(1979)) which provides a set of eigenvalues and their corresponding eigenvectors. The largest eigenvalue is the estimated source strength. The average of the remaining eigenvalues is the noise level. The eigenvector corresponding to the largest eigenvalue is crossed with the replica fields to get the estimated maximum correlation coefficient.

We once again use the linear array to test the characteristics of the new VCLM processor. The $150 \mathrm{~Hz}$ source is located in the far field with the incident wave modeled as a plane wave. The incident angle is 0 degrees and the average SNR at the receiver array is $10 \mathrm{~dB}$. In Figure 2-15(a), the ambiguity function for a BE processor(dashed line), an MLM processor(solid line), and an VCLM processor(dash-dotted line) are plotted. The VCLM ambiguity function appears to be missing though in reality it is merely being obscured by the MLM ambiguity function. This result occurs since there is an exact match between the source and the replica field at 0 degrees. The exponent of the VCLM processor is automatically selected as 1 by the optimization algorithm and the method reduces to the 

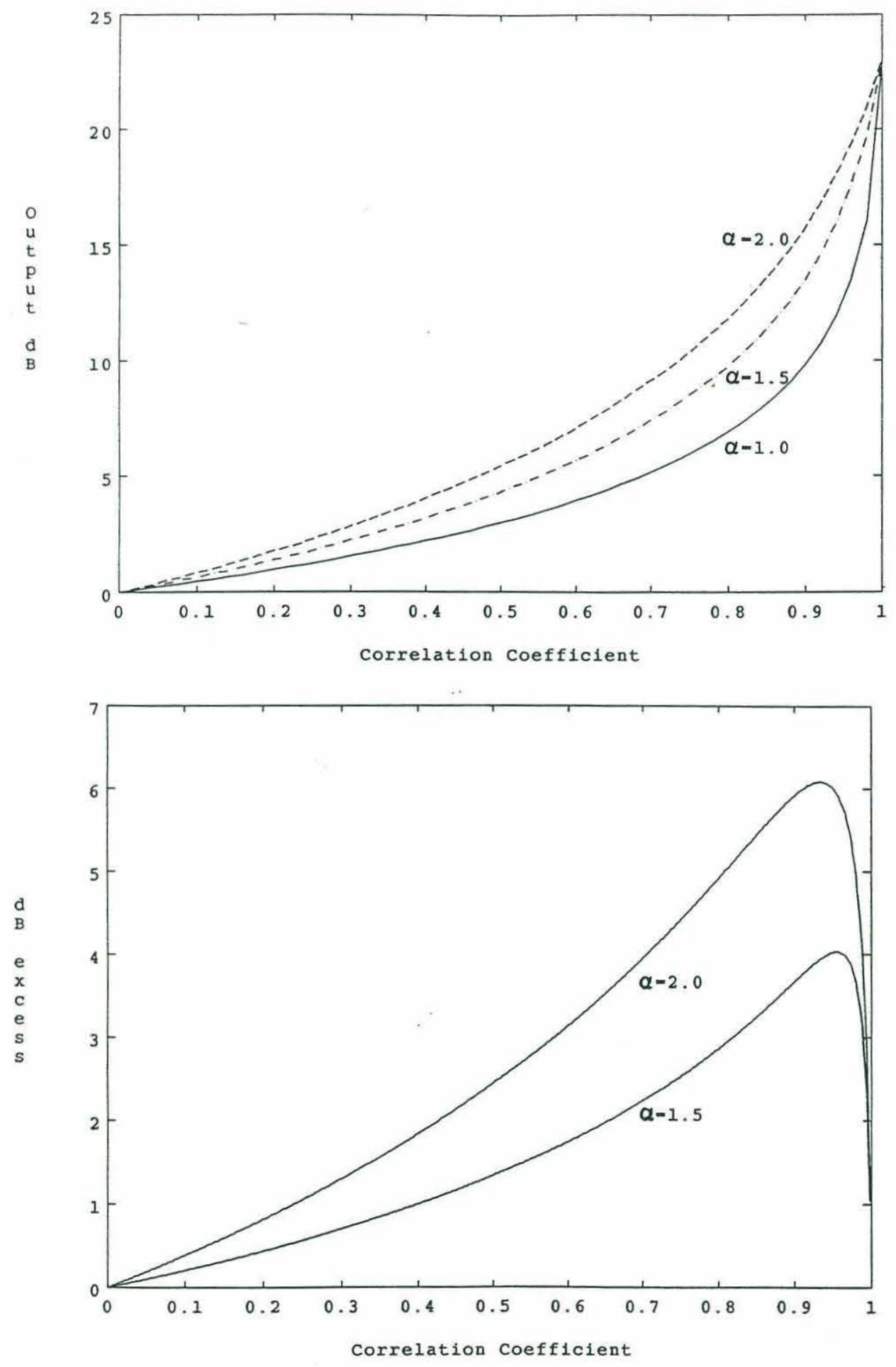

Figure 2-14: (a)Output of VCLM processor vs. correlation coefficient for exponent values of 1.0, 1.5, and 2.0. (b) Difference between MLM output and VCLM output vs. correlation coefficient for exponent values of 1.5 and 2.0 . 


\section{MLM processor.}

Next, the source is moved closer to the array (range $=1000$ meters) so that the incident field has a curvature which prevents a perfect match with any plane wave replicas. The incident angle remains 0 degrees and the signal strength is adjusted such that the average SNR at the receiver is still $10 \mathrm{~dB}$. As seen in Figure 2-13(b), the VCLM beamformer maintains a large value at the peak since the algorithm has selected a new exponent value which maximizes the peak relative to all other values. In general, the VCLM processor will always work better than the MLM processor and shows the most improvement at high SNR and low correlation between the source and replica fields.

Although these examples have used plane waves for illustrative purposes, the results are easily extraplolated to more complicated processes, as will be shown in our shallow water waveguide examples. 

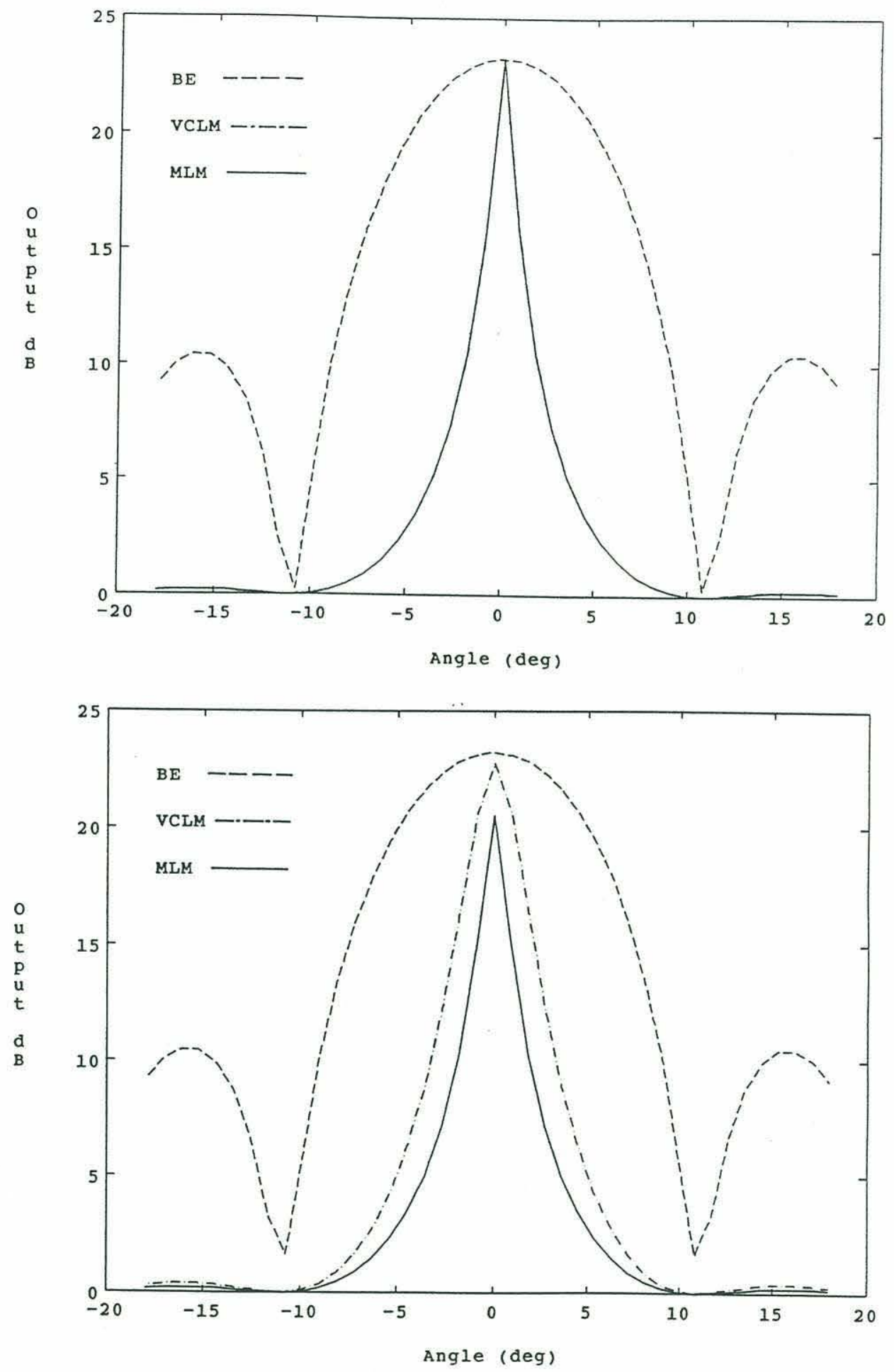

Figure 2-15: Plane wave ambiguity function for a BE processor(dashed line), an MLM processor(solid line), and an VCLM processor(dash-dotted line) in the case of (a) a perfect match and (b) a mismatch caused by plane wave assumption with source close to array. 


\section{Chapter 3}

\section{Analysis and Results}

\subsection{Estimation of the Covariance Matrix}

Estimation of the covariance matrix involves many issues and tradeoffs. We have selected the Fourier, or direct, method of spectral estimation (Kay and Marple, 1981). Below is a block diagram which shows the basic process. We briefly summarize our selection of sampling interval, window function, and data length.

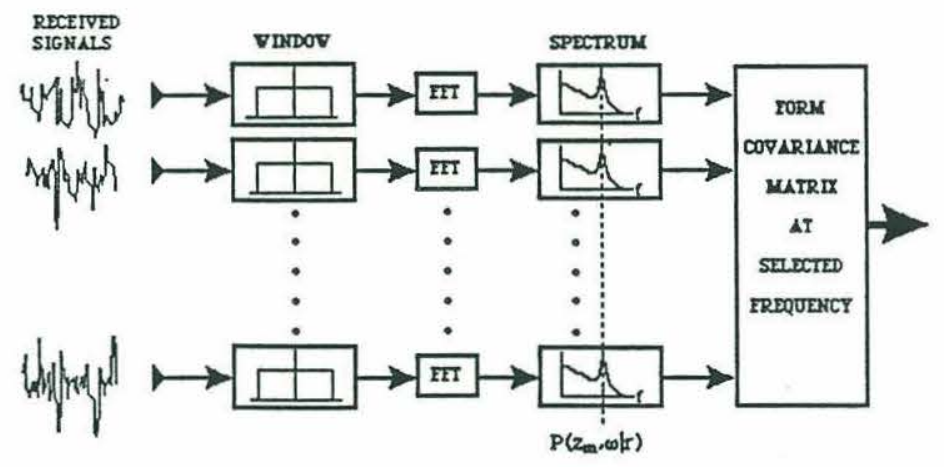

Given a $150 \mathrm{~Hz}$ signal, the Nyquist sampling requirements would be on the order of 1 msec. However, due to the narrowband assumption, we need only sample at the frequency required by the underlying process which we are studying (e.g. surface waves effects). In our study, the doppler shift caused by the moving source proves to be the limiting constraint of sampling frequency. We have selected a sample interval of 0.1 seconds which gives us a doppler window of $\pm 5 \mathrm{~Hz}$. This is sufficient for the doppler shift in our study.

Normally, the data must be multiplied by a window (e.g. Bartlett, Hamming, Hanning) 
to reduce sidelobe leakage (Harris, 1978). Given aprior knowledge of the single narrowband frequency, we due not have to worry about frequency interference and therefore have selected a rectangular window which provides us with the greatest resolution.

The selection of the data length was reflective of several constraints. First, a minimum time is required to get an accurate estimate of the covariance matrix with a low variance. The rough surface provided the minimum requirement on the order of several seconds. The maximum time was limited by 'smearing' effects caused by the moving source. As a result, a compromise of 12.8 seconds (128 data points) was selected.

\subsection{Surface Wave Effects}

\subsubsection{Analysis}

In our study of surface wave effects, we consider the localization of a $150 \mathrm{~Hz}$ omnidirectional point source in a Pekeris waveguide. The channel parameters are: $\rho_{\text {bottom }} / \rho_{\text {water }}=$ $1.772 ; c_{\text {water }}=1500 \mathrm{~m} / \mathrm{sec} ; c_{\text {bottom }}=1621.62 \mathrm{~m} / \mathrm{sec} ; H=100 \mathrm{~m}$. The receiver array consists of 21 hydrophones equally spaced from $25 \mathrm{~m}$ to $75 \mathrm{~m}$ ( $\lambda / 4$ apart). The average signal-to-noise ratio at the receiver $(+10 \mathrm{db})$ is selected to facilitate the study of the sea surface effect without undue noise interference.

The ambiguity surface is computed over a large grid to determine the magnitude of the source peak as well as quantify the background statistics. The grid size is chosen such that the ambiguity functions are sampled at the exact source position of $4 \mathrm{~km}$ in range and $54 \mathrm{~m}$ in depth. This is ensured by choosing 21 sampling points in the range interval 3 to $5 \mathrm{~km}(\Delta r=100 \mathrm{~m})$, and 21 points in the depth interval 10 to $90 \mathrm{~m}(\Delta z=4 \mathrm{~m})$. The peak-to-background ratio (PBR) expressed in $\mathrm{dB}$ is then calculated for the 'measured' data.

\subsubsection{Comparison of BE and MLM Processors in an Ideal Case}

We first analyze the performance characteristics of matched field processing using a $\mathrm{BE}$ processor and a MLM processor in a perfectly matched case. The propagation model used to synthetically produce the 'measured' signal is the same as that used to produce 
the replica fields, namely, a flat surface model. We have included a contour plot which shows the top ten $\mathrm{dB}$ of each ambiguity surface so that a better comparison of sidelobe suppression can be made.

The BE processor's ambiguity surface, as shown in Figure 3-1 has the global peak at the true source position but also has many large ambiguous peaks. The magnitude of the source peak is $24.5 \mathrm{~dB}$ but mainly as a result of increased background levels, the peak-tobackground ratio is only $12 \mathrm{~dB}$. In Figure 3-2, the MLM processor produces an ambiguity surface that clearly displays the true source location with a peak magnitude of $24.5 \mathrm{~dB}$ above the background and all ambiguous peaks reduced by more than $10 \mathrm{~dB}$. In fact, the largest ambiguous peak is $19 \mathrm{~dB}$ less than the source peak. Throughout our study, the $\mathrm{BE}$ processor could not unambiguously locate the source, and so for the remainder of the thesis, its results will not be displayed.

\subsubsection{Effects of increasing wave heights on the MLM estimator}

The sensitivity to surface wave height mismatch is now investigated. The replica fields are generated using the same flat surface model as before. However, the 'measured' field is computed using a model which incorporates the surface wave scattering as discussed in Section 2.2. An absolute measure of processor sensitivity cannot be performed here since the process is highly non-linear, yet we can select characteristic environmental conditions which will illustrate the effect of varying surface wave heights.

For each of nine different wave heights (from 0 to 2 meters rms), we generate a 150 $\mathrm{Hz}$ signal along the receiver array. The cross-spectral covariance matrix is estimated and used as input to the MLM processor based on a replica model field using a flat surface. As an example, Figure 3-3 and Figure 3-4 illustrate the cases of 0.5 and 1.0 meter rms wave height, respectively. For 0.5 meter surface wave height, the main peak exhibits a $7.0 \mathrm{~dB}$ reduction compared to the flat surface case. For the larger wave height of 1.0 meter, the main peak experiences a $11.6 \mathrm{~dB}$ decrease from the ideal case. In general, when the wave height is small, the source location is easily identifiable. As wave height increases, the source location peak decreases and ultimately is indistinguishable from ambiguous peaks at erroneous source locations. 


\section{Ideal Case (BE)}
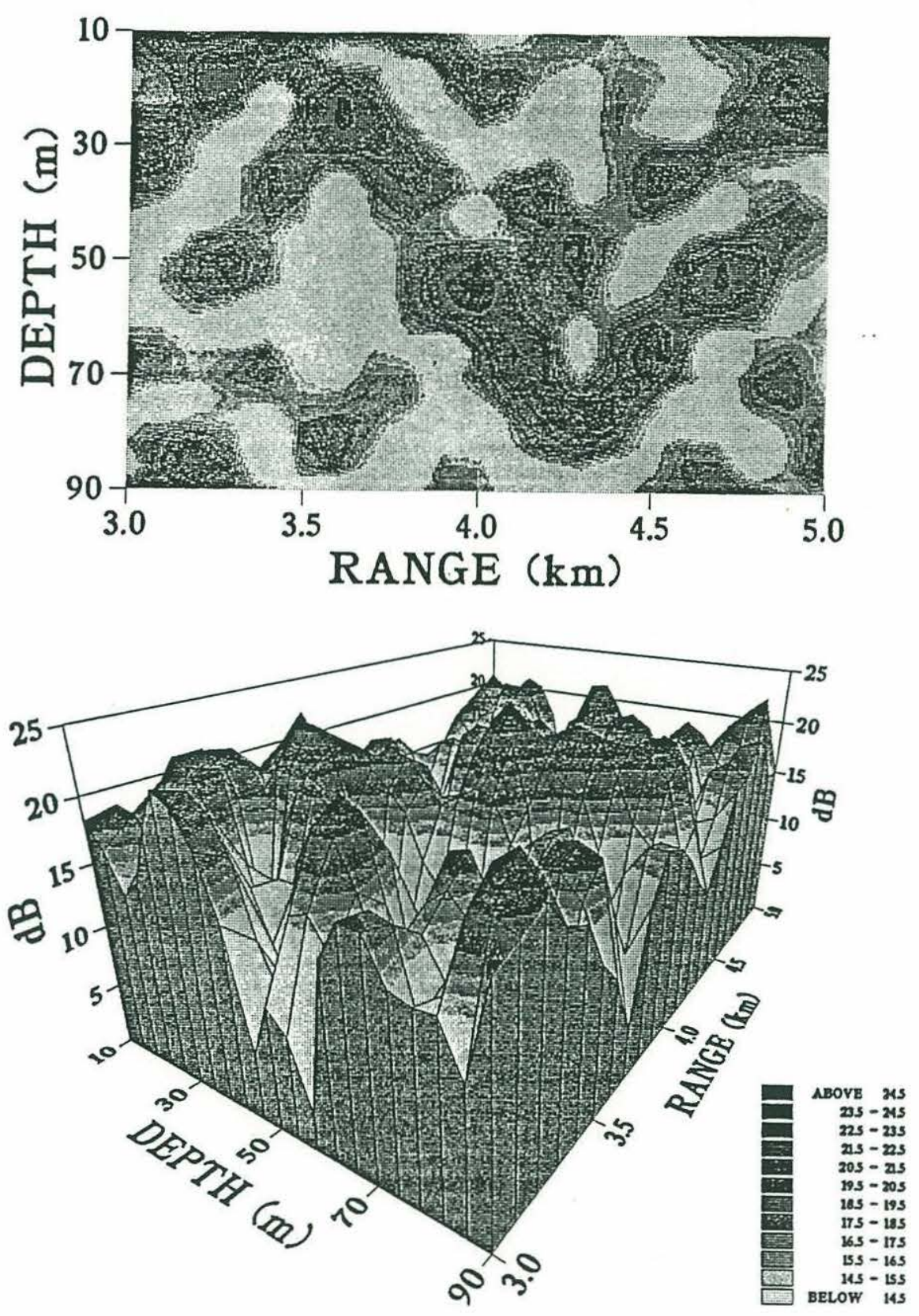

Figure 3-1: Ambiguity surface using a BE processor in an ideal case. True source location is at a range of $4 \mathrm{~km}$ and depth of $54 \mathrm{~m}$. 


\section{Ideal Case (MLM)}
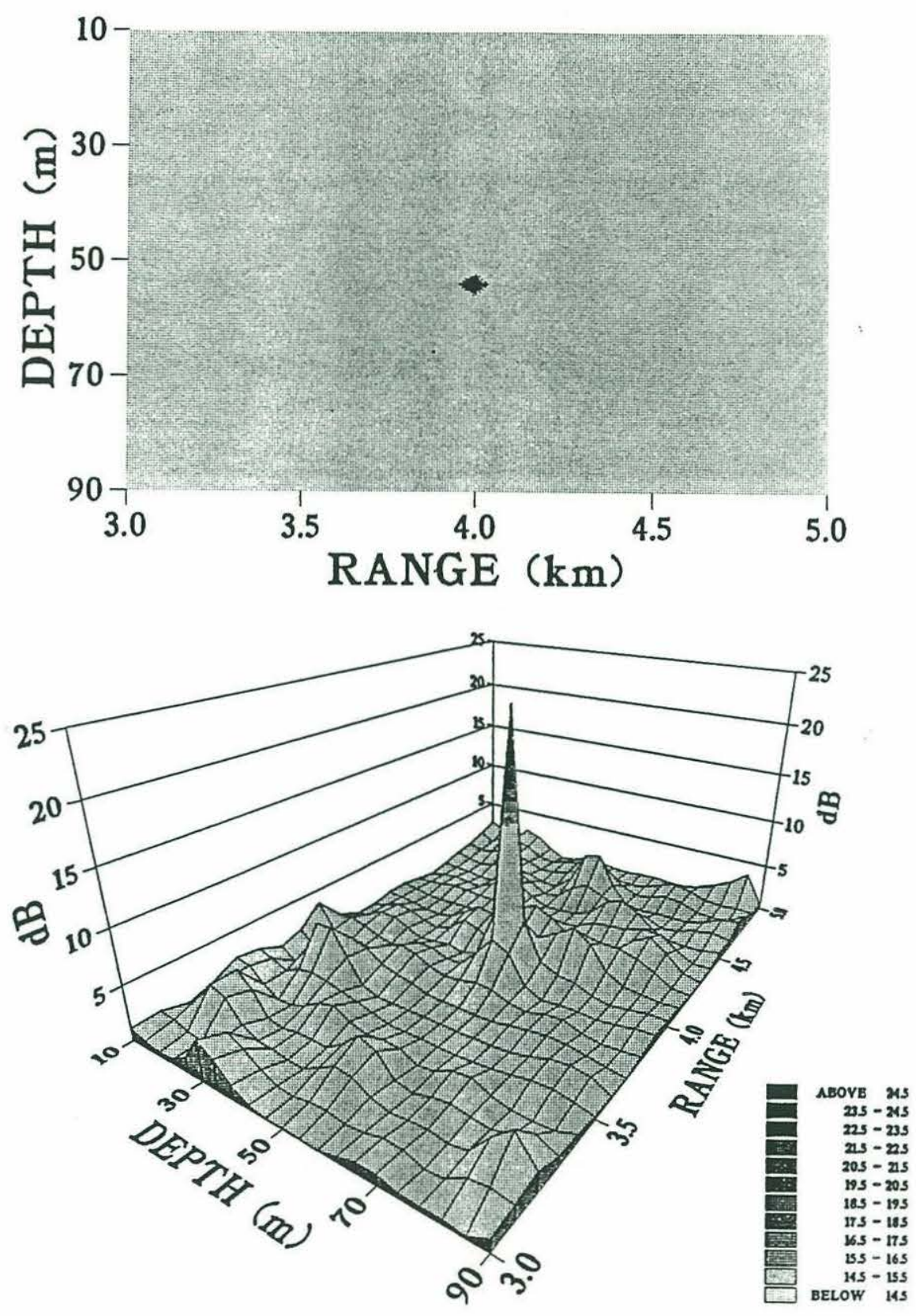

Figure 3-2: Ambiguity surface using an MLM processor in an ideal case. True source location is at a range of $4 \mathrm{~km}$ and depth of $54 \mathrm{~m}$. 


\section{Surface Waves $(0.5 \mathrm{~m})$}
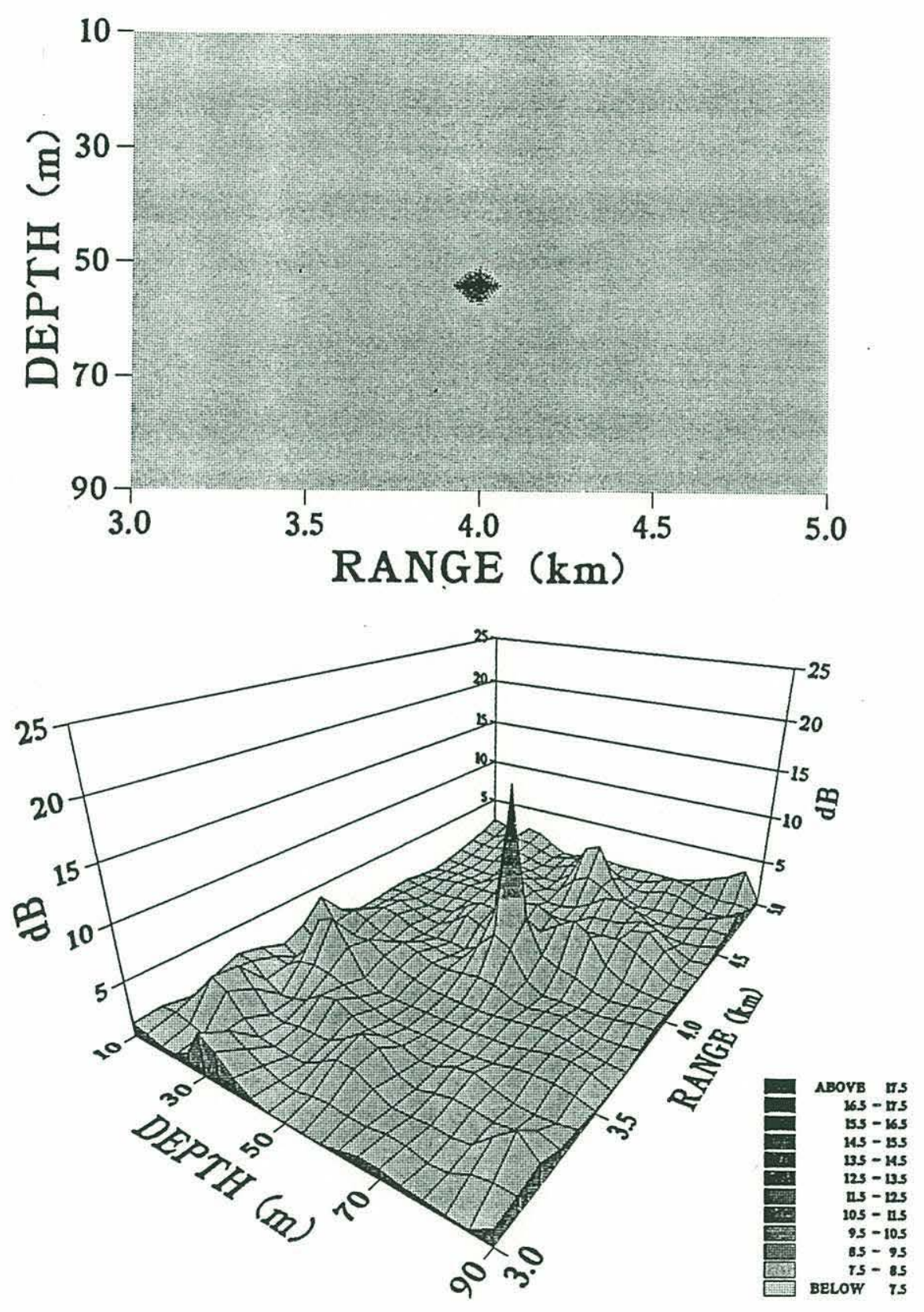

Figure 3-3: MLM ambiguity function for mismatched surface condition of $0.5 \mathrm{~m}$ rms wave height. True source location is at a range of $4 \mathrm{~km}$ and depth of $54 \mathrm{~m}$. 


\section{Surface Waves $(1.0 \mathrm{~m})$}
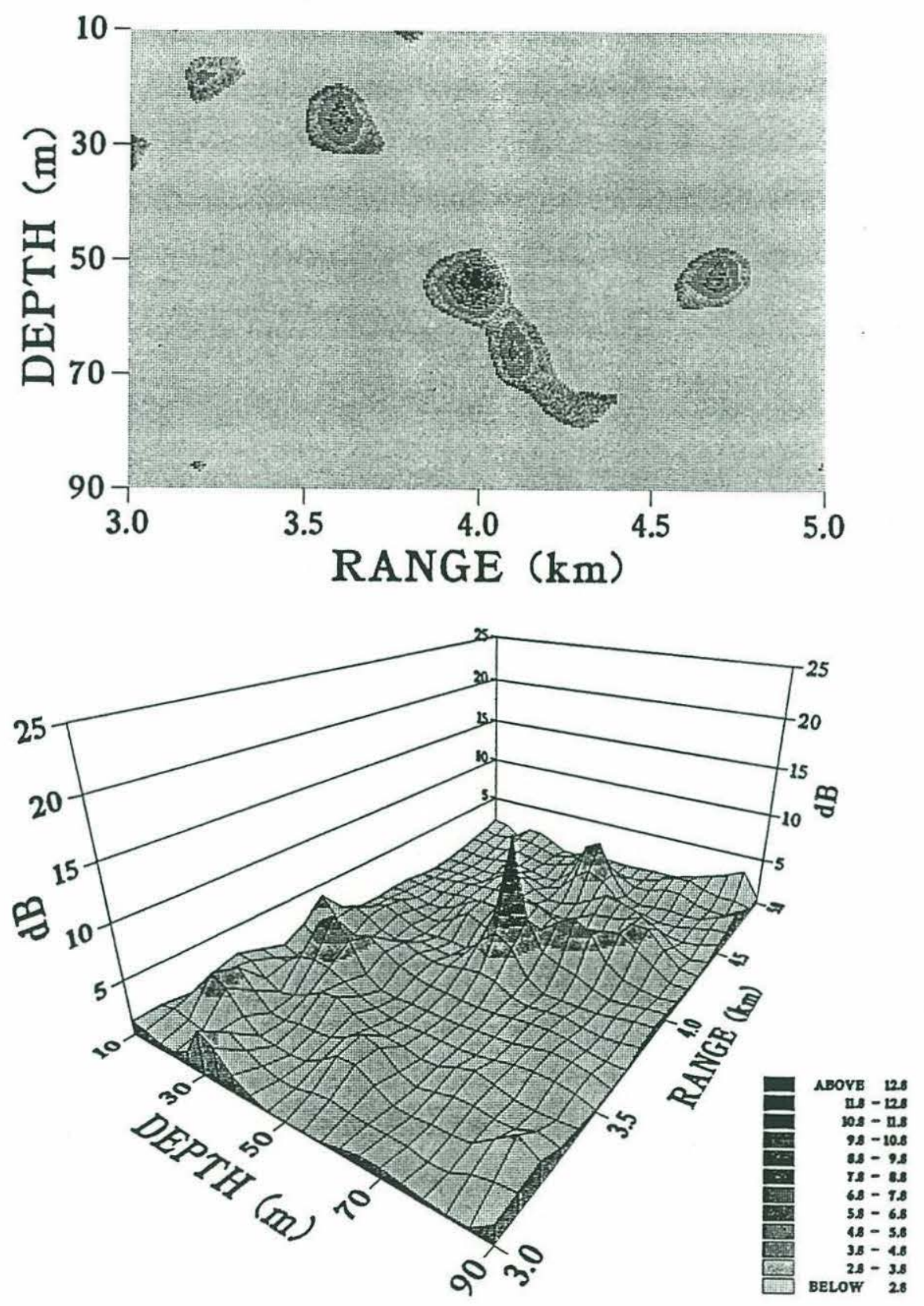

Figure 3-4: MLM ambiguity function for mismatched surface condition of $1.0 \mathrm{~m}$ rms wave height. True source location is at a range of $4 \mathrm{~km}$ and depth of $54 \mathrm{~m}$. 


\subsubsection{Counteracting surface wave effects}

The problem thus posed is to develop a method which will counteract the deleterious effects of a randomly introduced sea surface error on the signal processing. One could naively reason that increasing the time window of the 'measured' signal would result in the averaged signal at the random surface converging to the flat surface model. Yet, even an 'infinite-time' signal provides only a slight increase in PBR over the finite time signal and is still much lower than the flat surface maximum. The reason for this can be found with a little math and some simple probability theory. By taking the expectation of the coherent reflection for a finite width beam we discover that it is equal to the Eckart coherent reflection coefficient, i.e.,

$$
E\left[\prod \int_{s} e^{-i 2 \gamma_{1} \xi(s)} d s\right]=\prod E\left[\int_{s} e^{-i 2 \gamma_{1} \xi(s)}\right]=\prod e^{-2 \gamma_{1}^{2} \sigma^{2}}
$$

In other words, the coherent reflection from a rough surface can be treated as an incomplete reflection from a flat surface. Therefore, the infinite-time pressure field does not converge to the flat surface replica model with perfect reflection. Consequently, increasing the time window of the measured signal does not result in greater correlation to the flat surface model.

On the other hand, by using the Eckart coherent reflection coefficient based on the actual sea surface statistics, replica fields can be generated to which the 'infinite-time' cross spectral covariance matrix will converge. The time period for convergence to occur is found to be on the order of the period of the surface waves. The following results were based on 12.8 seconds of data. In Figure 3-5 and Figure 3-6, we again illustrate the ambiguity surfaces generated for surface wave heights of 0.5 and 1.0 meters rms, respectively, but this time using replica fields constructed with matching sea surface statistics. For 0.5 meter wave height, there is a $6.5 \mathrm{~dB}$ gain by matching the sea surface statistics. For the 1.0 meter wave height, there is a $10.2 \mathrm{~dB}$ gain. In both cases, the new peak is within 1 $\mathrm{dB}$ of the perfectly matched flat surface model.

The complete results of matching the model to the actual statistical sea surface conditions are illustrated in Figure 3-7. Based on the sea surface statistics of 0.0, 0.5, and 1.0 meters, we generated three different model fields. We have previously generated 9 


\section{Surface Waves $(0.5 \mathrm{~m})$}
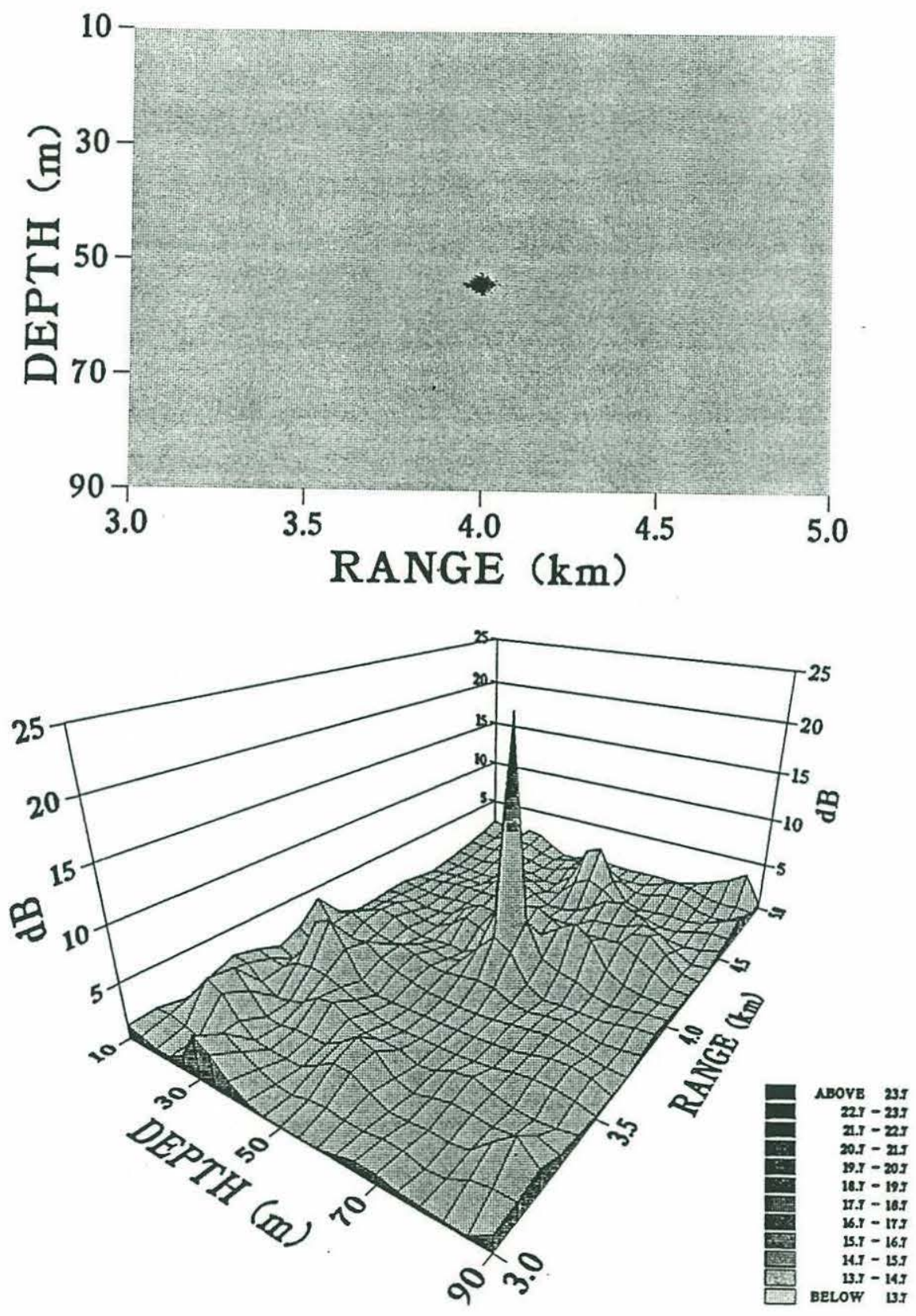

Figure 3-5: MLM ambiguity function for matched surface condition of $0.5 \mathrm{~m}$ rms wave height. True source location is at a range of $4 \mathrm{~km}$ and depth of $54 \mathrm{~m}$. 


\section{Surface Waves $(1.0 \mathrm{~m})$}
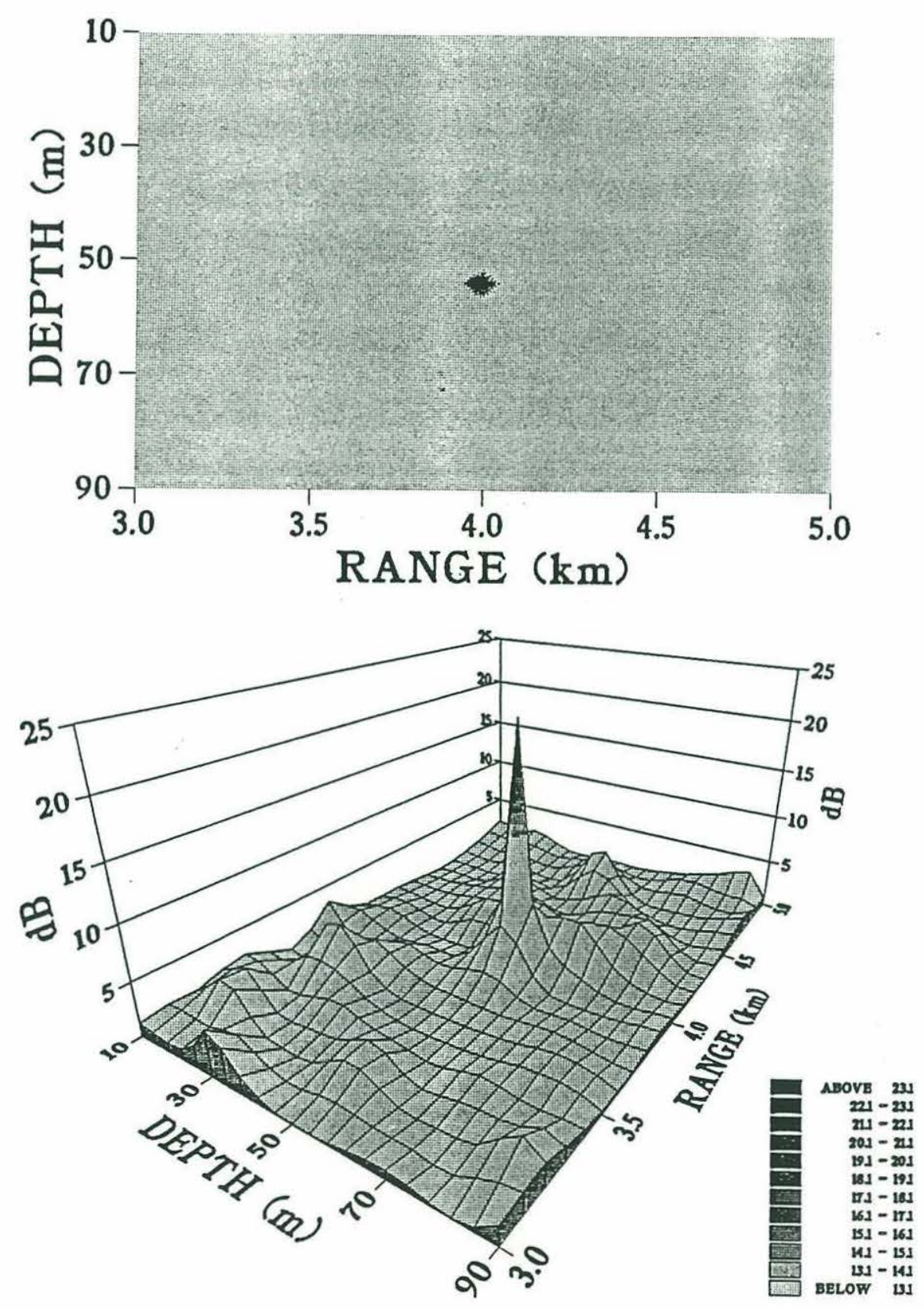

Figure 3-6: MLM ambiguity function for matched surface condition of $1.0 \mathrm{~m}$ rms wave height. True source location is at a range of $4 \mathrm{~km}$ and depth of $54 \mathrm{~m}$. 
sets of 'measured' data, one for each $1 / 4$ meter rms wave height up to 2 meters. The output of the MLM processor is calculated for each combination of the 'measured' data and the model field (27 total) and the PBR for each case is plotted (three solid lines, one for each model). Also annotated on the plot are the specific cases which have previously been displayed. As observed, the PBR is maximized when the model sea surface statistics match the actual sea surface conditions. The dashed line formed by the boundary of the solid curves is the maximum PBR for a given wave height, and will be referred to as the Eckart Limit. It is interesting to note that this limit decreases with wave height even though 'perfect' correlation between the actual signal and the statistically matched model can be obtained. The variance of the sea surface that resulted in phase interference while coherently averaging, ultimately results in reduced signal strength at the receiver. The smaller SNR causes the decreased PBR.

\subsection{Internal Wave Effects}

\subsubsection{Analysis}

In our investigation of internal wave effects on matched field processing, the same environmental parameters are used as in the surface wave study with the following exceptions. The upper 20 meters of the water channel is modeled as a warm surface layer such that the speed of sound has increased $5.0 \mathrm{~m} / \mathrm{sec}$ and the density has decreased by $.001 \mathrm{gm} / \mathrm{cc}$ from the background water channel. The source is moved across the layer to a depth of 14 meters. The grid spacing has been decreased to accomodate the requirements of the MCM processor which will be used with this model when we look at the combined effects of surface waves, internal waves, and moving sources. There are now 41 grid points in the range interval 3 to $5 \mathrm{~km}(\delta r=50 \mathrm{~m})$ and 21 grid points in the depth interval 10 to 90 meters $(\delta z=4 \mathrm{~m})$.

\subsubsection{Effects of single frequency internal wave on the MLM estimator}

The replica fields are generated using a flat interface model. The 'measured' field is computed for a single frequency internal wave as discussed in Section 2.3. The internal 


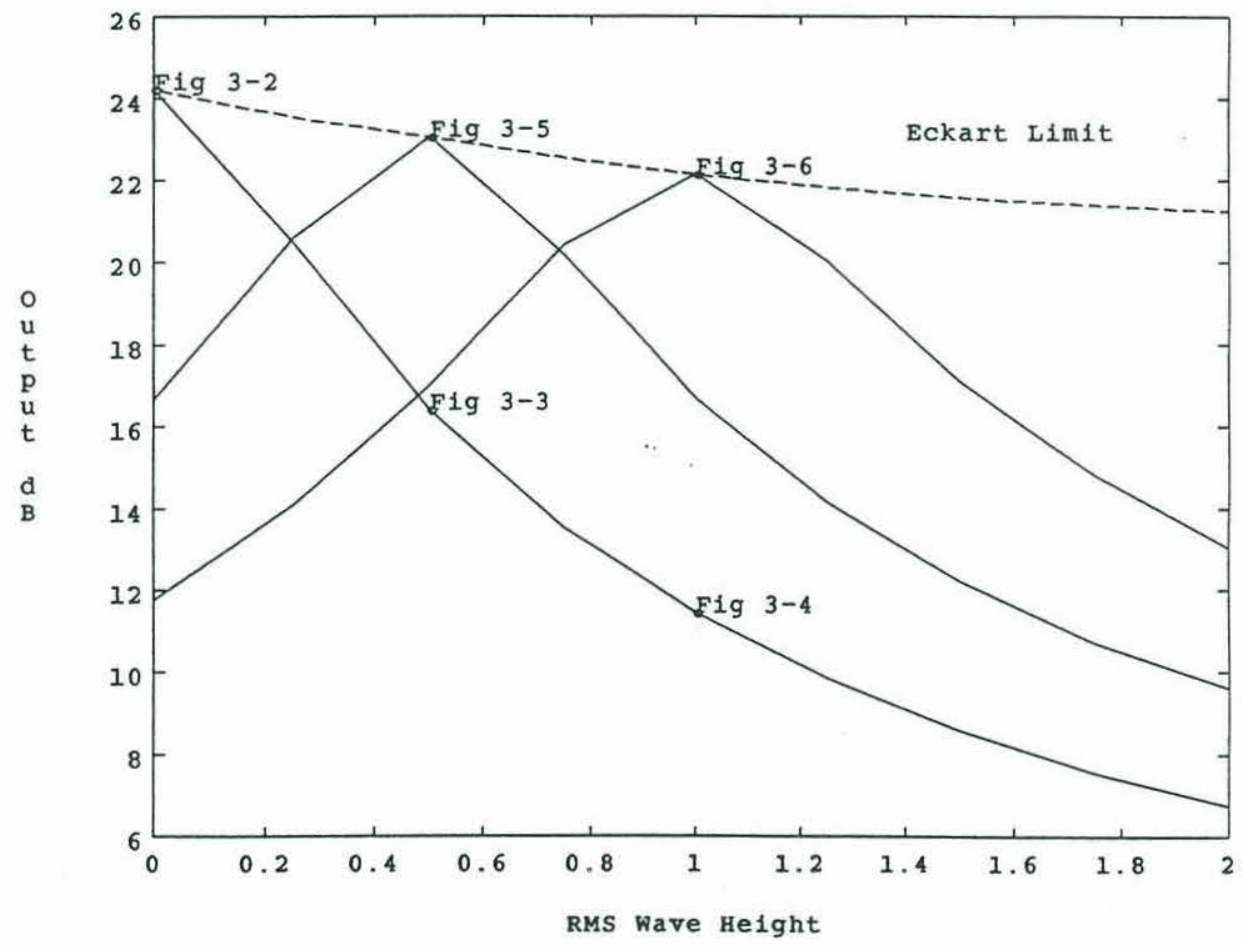

Figure 3-7: The effect on peak-to-background ratios for various surface wave height models vs. actual wave height 
wave is located at the discontinuity between the water layers, and has an amplitude of 2 meters, a wavelength of 200 meters, and a period of 10 minutes. Figure 3-8 illustrates the ambiguity surface which would be generated in the absence of the internal wave. The location of the source is clearly indicated with a peak magnitude of $24 \mathrm{~dB}$ above background. Figure 3-9 is generated with the internal wave present. As shown, the peak decreases by $11 \mathrm{~dB}$ from the ideal case.

\subsubsection{Counteracting internal wave effects}

In the surface wave study, we were able to regain almost all of the source peak by short time averaging of the measured signal on the order of a wave period and then correlating the results against a model which has a surface reflection coefficient that is dependent only on the average surface roughness and not the actual shape of the sea surface. For internal waves, neither of these techniques will work. First, the time period of internal waves is on the order of 10 minutes to several hours. This time scale prevents any resonable time averaging since many other ocean features as well as source position would also be changing. Secondly, the gaussian nature of the sea surface allowed us to describe it completely by its second moment and construct a sea surface model based on this statistic to which the measured data quickly converges. The internal wave is 'less random' in nature over the time and length scales used in the estimation of the covariance matrix and therefore cannot be described by a single statistic. That is, we are essentially bound to one realization of a random process. Thus, we must not only know the amplitude of the internal wave but also its exact phase and phase speed as it propagates along the interface.

As an example, we calculate the output of the MLM processor at the source peak as a function of phase error (we assume the amplitude is known exactly) of the internal wave. As shown in Figure 3-10, when the phase is known exactly, the ideal PBR of $24 \mathrm{~dB}$ is obtained. But as the phase estimate becomes more in error, the PBR quickly decreases. The same would be true of an amplitude mismatch even if the phase is known. 


\section{Internal Wave $(0.0 \mathrm{~m})$}
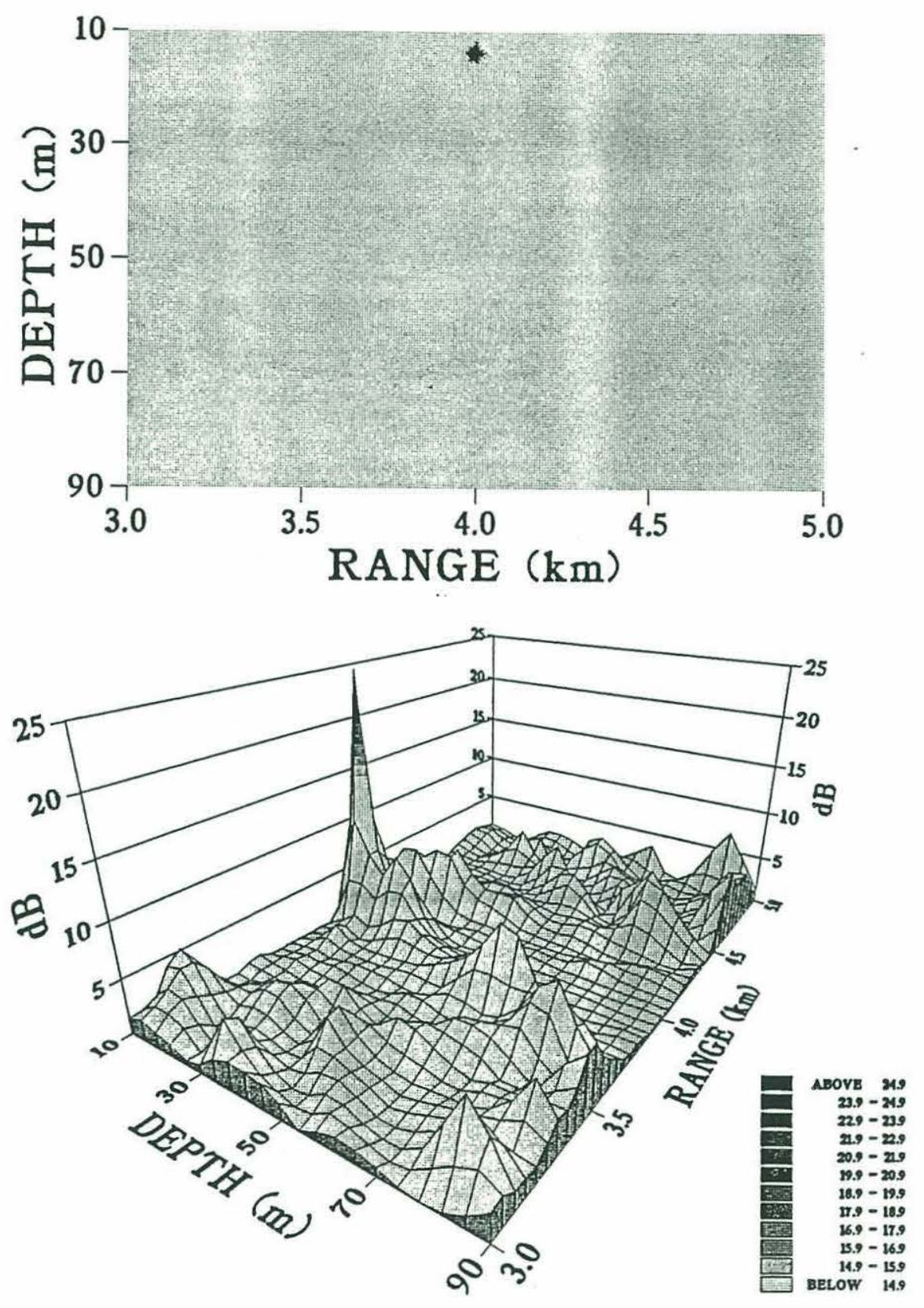

Figure 3-8: MLM ambiguity function for internal wave condition of $0.0 \mathrm{~m}$ amplitude internal wave height. True source location is at a range of $4 \mathrm{~km}$ and depth of $14 \mathrm{~m}$. 


\section{Internal Wave $(2.0 \mathrm{~m})$}
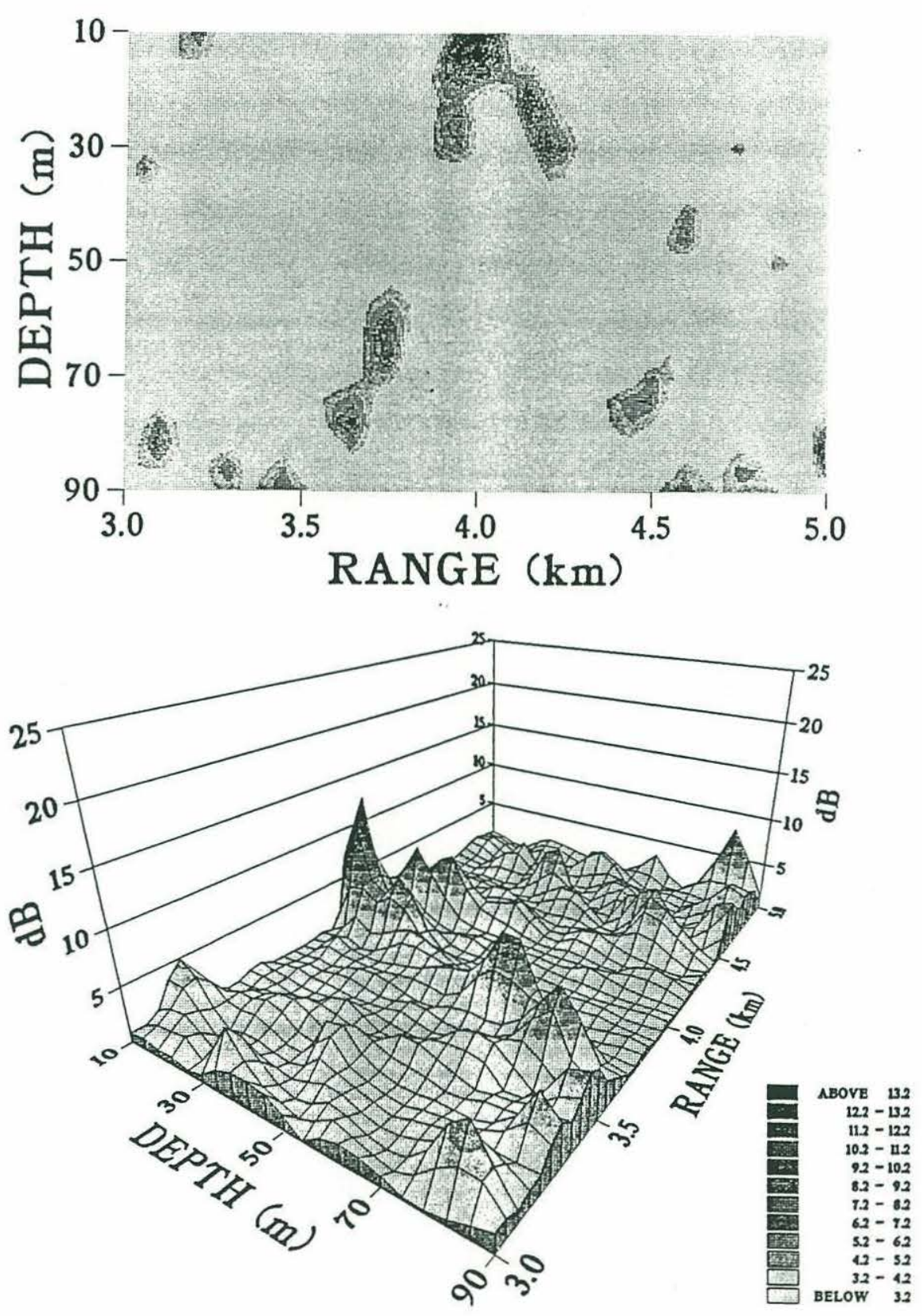

Figure 3-9: MLM ambiguity function for internal wave condition of $2.0 \mathrm{~m}$ amplitude internal wave height. True source location is at a range of $4 \mathrm{~km}$ and depth of $14 \mathrm{~m}$. 


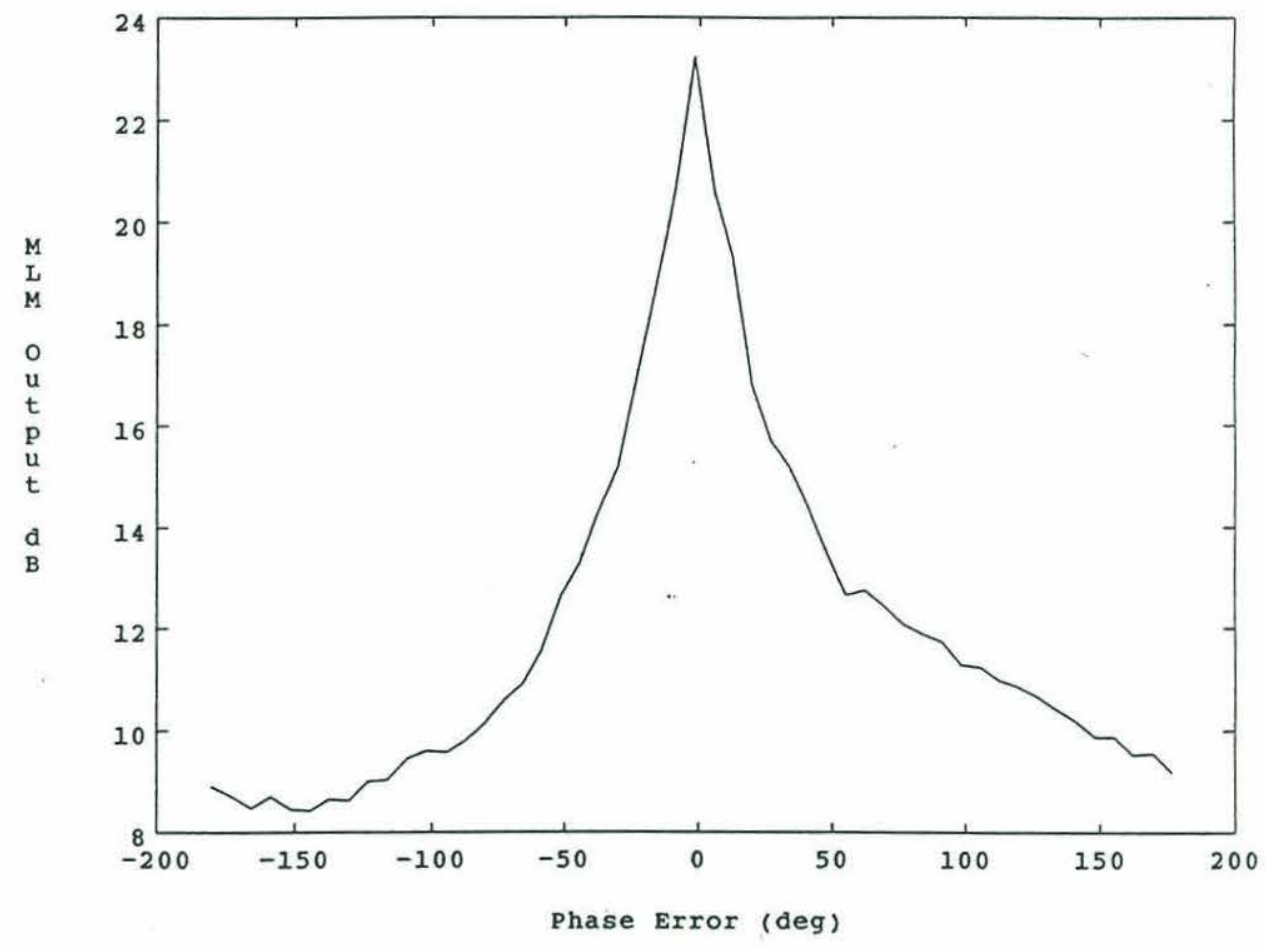

Figure 3-10: PBR as a function of phase estimation error for internal wave. 


\subsection{Moving Source Effects}

\subsubsection{Analysis}

We again use the three layer model as for the study of internal wave effects. The surface and internal wave models are not activated so that the effect of only the source motion can be studied. The source is positioned at a depth of 14 meters and a range of 4000 meters at time $t=0.0$ seconds. We study three cases in which the source is moving at speeds of 5,10 , and 15 knots toward the receiver. The acoustic signal undergoes a doppler shift as well as frequency spreading. We assume that the base frequency of 150 $\mathrm{Hz}$ is known and shift the spectrum accordingly. If the base frequency was not known, then we would either have to increase the scanning space to include frequency or else accept the additional mismatch between the received frequency and the actual frequency.

\subsubsection{Results from Moving Source Effects}

In Figure 3-11, the MLM ambiguity surface is displayed for a 5 knot target. The source peak has a value of $19.4 \mathrm{~dB}$ and no ambiguous peaks are present. This should be compared to Figure 3.8 in which the target is stationary. Even a slowly moving source experiences over $5 \mathrm{~dB}$ decrease at the peak. In Figure 3-12 the MLM ambiguity surface is displayed for a 10 knot source. The source peak has decreased to $15.1 \mathrm{~dB}$ and been extended in the direction of motion as would be expected. Ambiguous peaks have also appeared. In Figure 3-13 the MLM ambiguity surface is displayed for a 15 knot source. In this case, the source peak has decreased to $14.9 \mathrm{~dB}$ and the ambiguous peaks are even more evident.

\subsection{Combined Effects and Sub-optimal Processors}

\subsubsection{Analysis}

In order to get a more realistic view of the robustness of the various processors, we combine a slowly moving source with our surface and internal wave models to create a complete, time varying, shallow water model. The source is positioned at a range of 


\section{Moving Source $(5 \mathrm{kts})$}
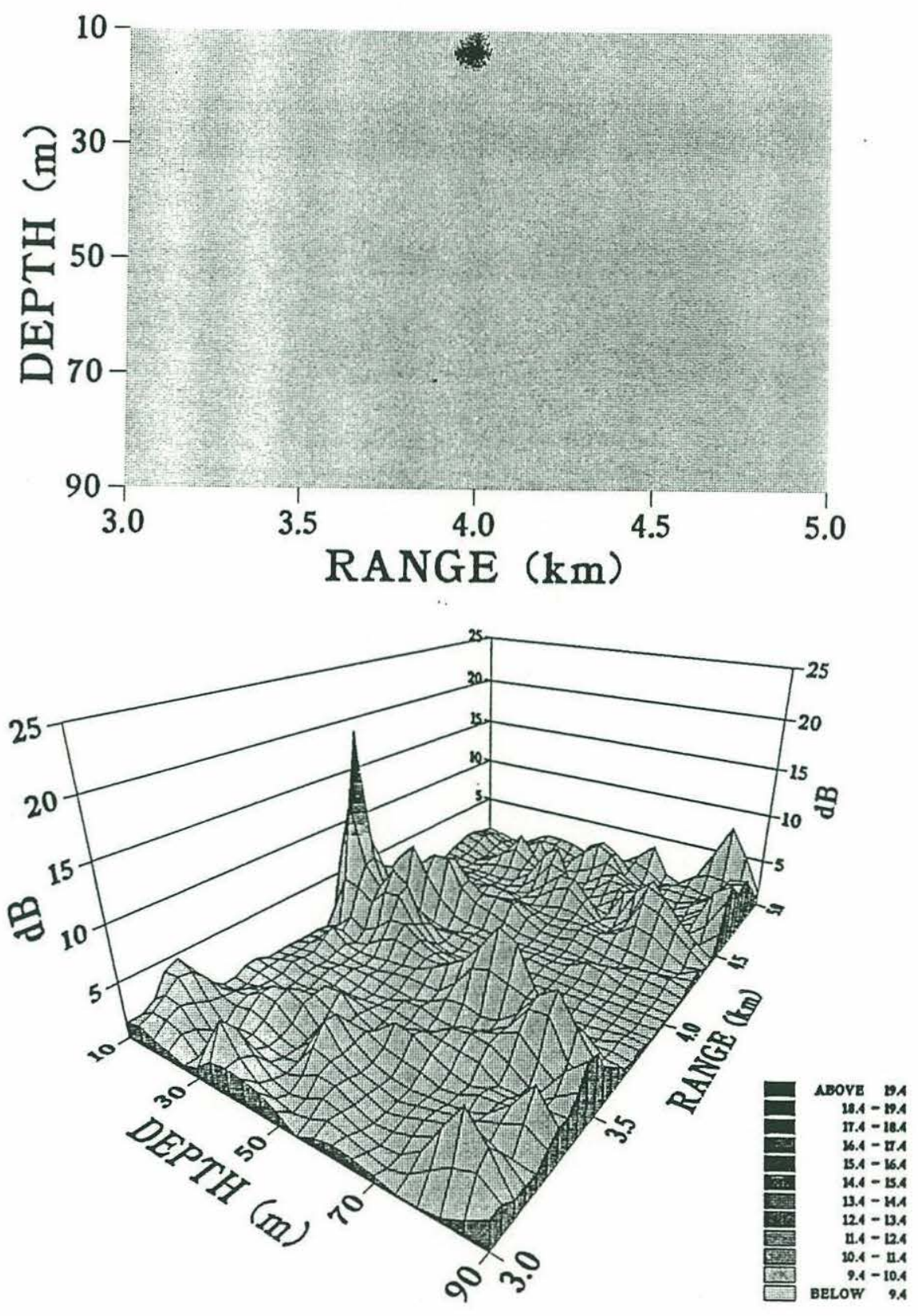

Figure 3-11: MLM ambiguity function for moving source effects. True source location is at a range of $4 \mathrm{~km}$ and depth of $14 \mathrm{~m}$ at $\mathrm{t}=0.0$, speed 5 knots toward receiver. 


\section{Moving Source (10kts)}
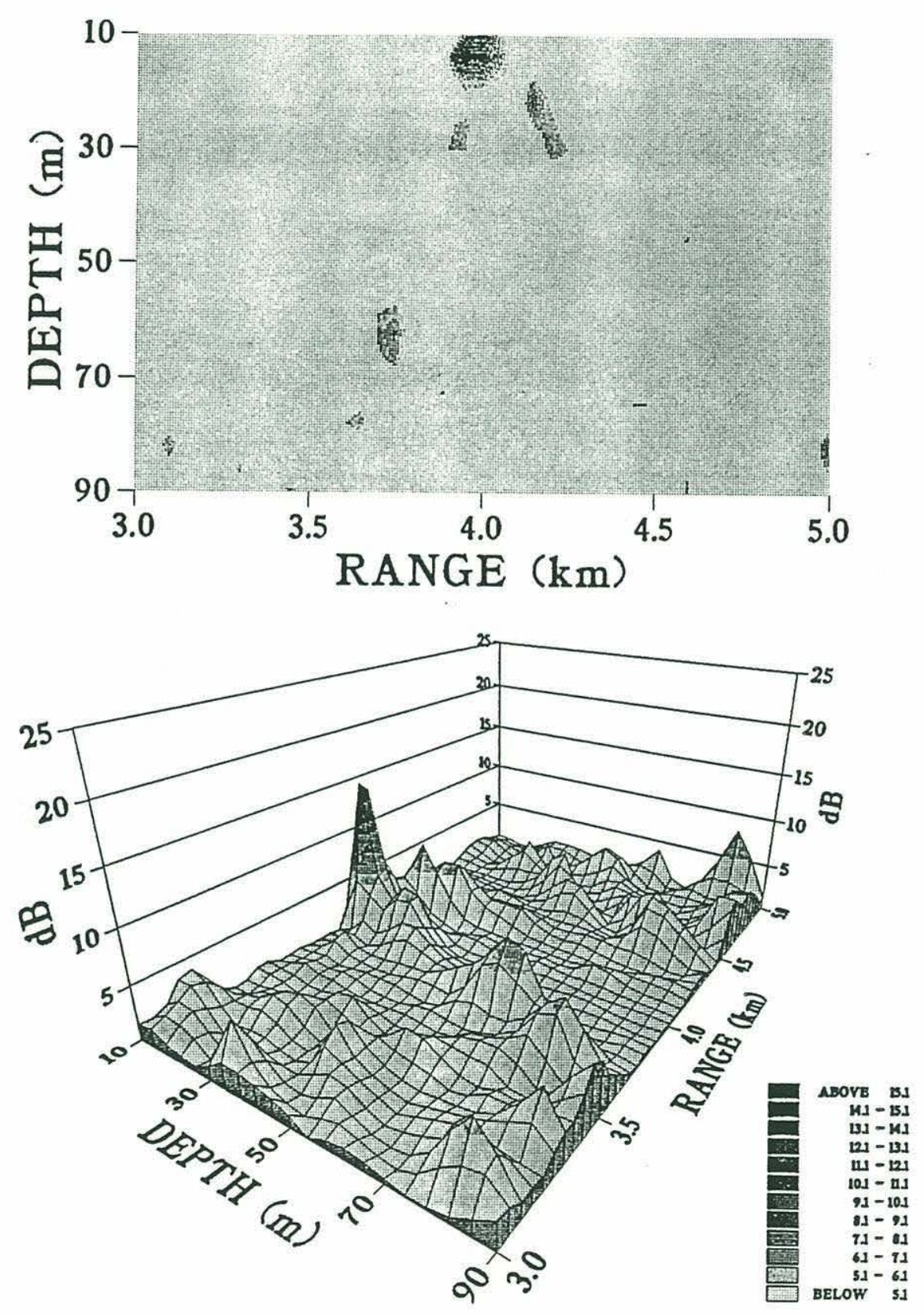

Figure 3-12: MLM ambiguity function for moving source effects. True source location is at a range of $4 \mathrm{~km}$ and depth of $14 \mathrm{~m}$ at $\mathrm{t}=0.0$, speed 10 knots toward receiver. 


\section{Moving Sources (15kts)}
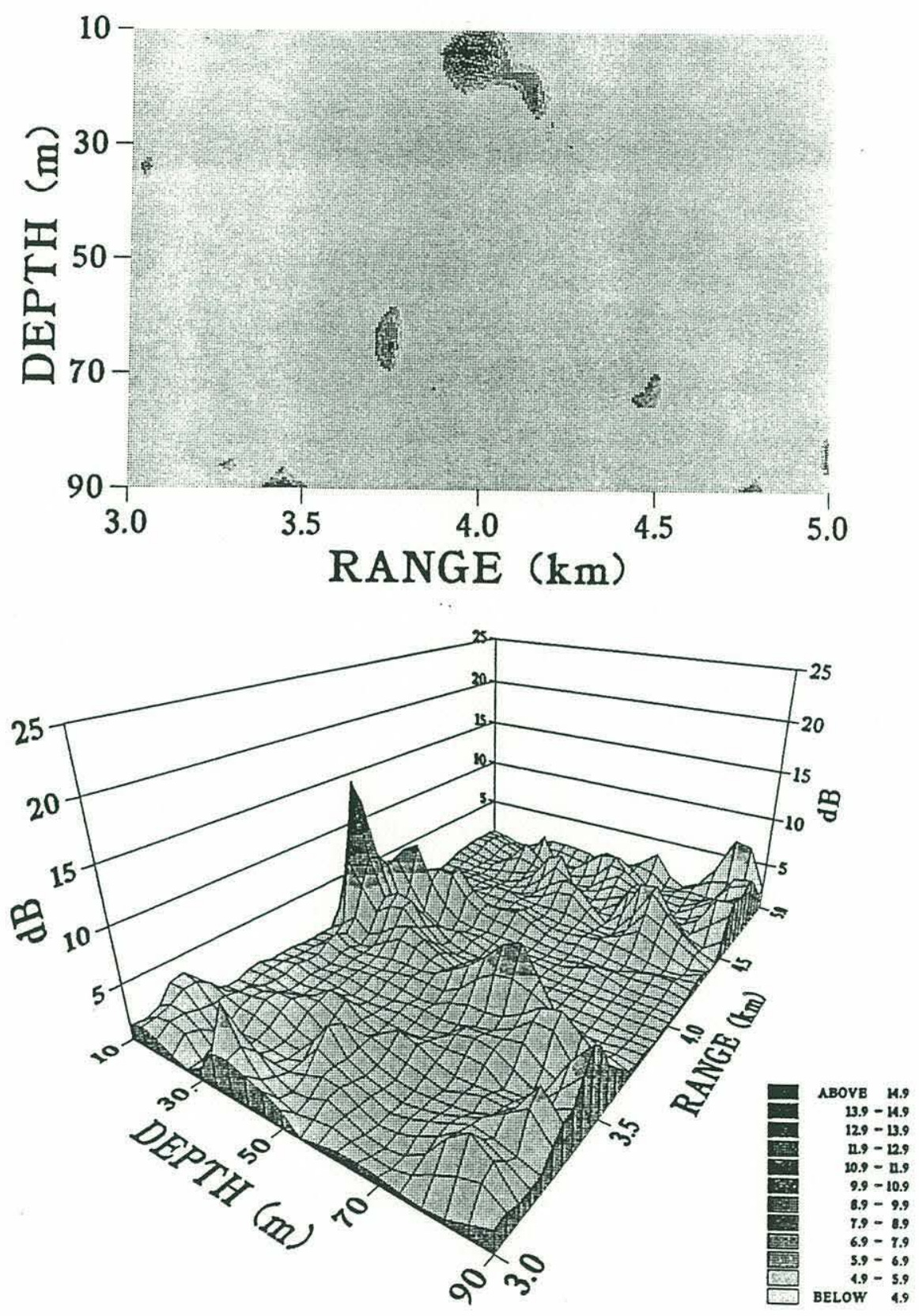

Figure 3-13: MLM ambiguity function for moving source effects. True source location is at a range of $4 \mathrm{~km}$ and depth of $14 \mathrm{~m}$ at $\mathrm{t}=0.0$, speed 15 knots toward receiver. 
4000 meters and a depth of 14 meters at time $t=0.0$. The source is moving at a speed of 5 knots toward the receiver. The surface wave model is activated with a sea surface variance of 1.0 meter. The internal wave model is activated with a 2 meter amplitude wave of unknown phase. The covariance matrix is constructed from this data and used as input into the various processors. The predicted fields are created using the same three layer model, including incomplete reflection at the sea surface matched to the surface roughness. Grid spacing remains the same as in the previous section.

\subsubsection{Results from Combined Effects}

We now analyze the results using three processors; MLM, MCM and VCLM. In Figure 3-14 the MLM ambiguity surface is displayed. As expected, the source peak has decreased from a maximum of $24 \mathrm{~dB}$ for the perfectly matched case to a value of $11.0 \mathrm{~dB}$. In addition, there are many ambiguous peaks evident. For the MLM processor, the signal is too highly matched to a single replica and as a result, we would be unable to locate the source with any sort of confidence.

In a realistic ocean environment, a more robust processor is called for. Using the same data, we now implement the MCM processor. In the plane wave example, the actual complexity as well as the importance of the grid selection was transparent. So long as the expanded grid area was within the mainlobe, the algorithm worked exceptionally well. In our shallow water waveguide, the field is much more complex with the width of the mainlobe varying throughout the scanning region. Thus, the optimal grid selection varies, depending on the true source location in combination with the model parameters. The original 21 by 21 grid used for surface waves was inadequate, and resulted in a degraded solution. This required an increase in the grid locations as reflected by the 41 by 21 grid $(\delta r=50 \mathrm{~m} ; \delta z=4 \mathrm{~m})$ used for all the two layer waveguide examples. The expanded grid area includes the postulated grid location as well as its closest neighbor in range and depth on either side for a total of 5 grid points. On the edge of the ambiguity surface the grid area has been reduced accordingly. Grid spacing as close as $\delta r=4 \mathrm{~m}$ and $\delta z=0.5 \mathrm{~m}$ provided little improvement in source peak output while significantly increasing computational time. 
The MCM ambiguity surface is displayed in Figure 3-15. Due to its more robust nature, the source peak has increased in value from $11.0 \mathrm{~dB}$ to $17.0 \mathrm{~dB}$. However, the expanded grid area results in the 'widening' of the ambiguity peaks and poorer resolution. In addition, although there has been some suppression of ambiguous peaks, the peak at a depth of 70 meters and a range of 3700 meters is comparable to the source peak. Even worse, the secondary peak has increased more than the main peak. In general, for cases with little mismatch, we found this to be the rule rather than the exception.

Using a VCLM processor, the data are again analyzed with the results displayed in Figure 3-16. The source peak has now increased to $19.4 \mathrm{~dB}$. More importantly, the source peak has increased relative to all ambiguous peaks, i.e., one has much better sidelobe suppression. Furthermore, the optimal resolution of the VCLM processor is not sacrificed to achieve this robustness. As a result, the VCLM processor provides a much better estimation of the source location than either the MLM or MCM processor. 


\section{Combined Effects (MLM)}
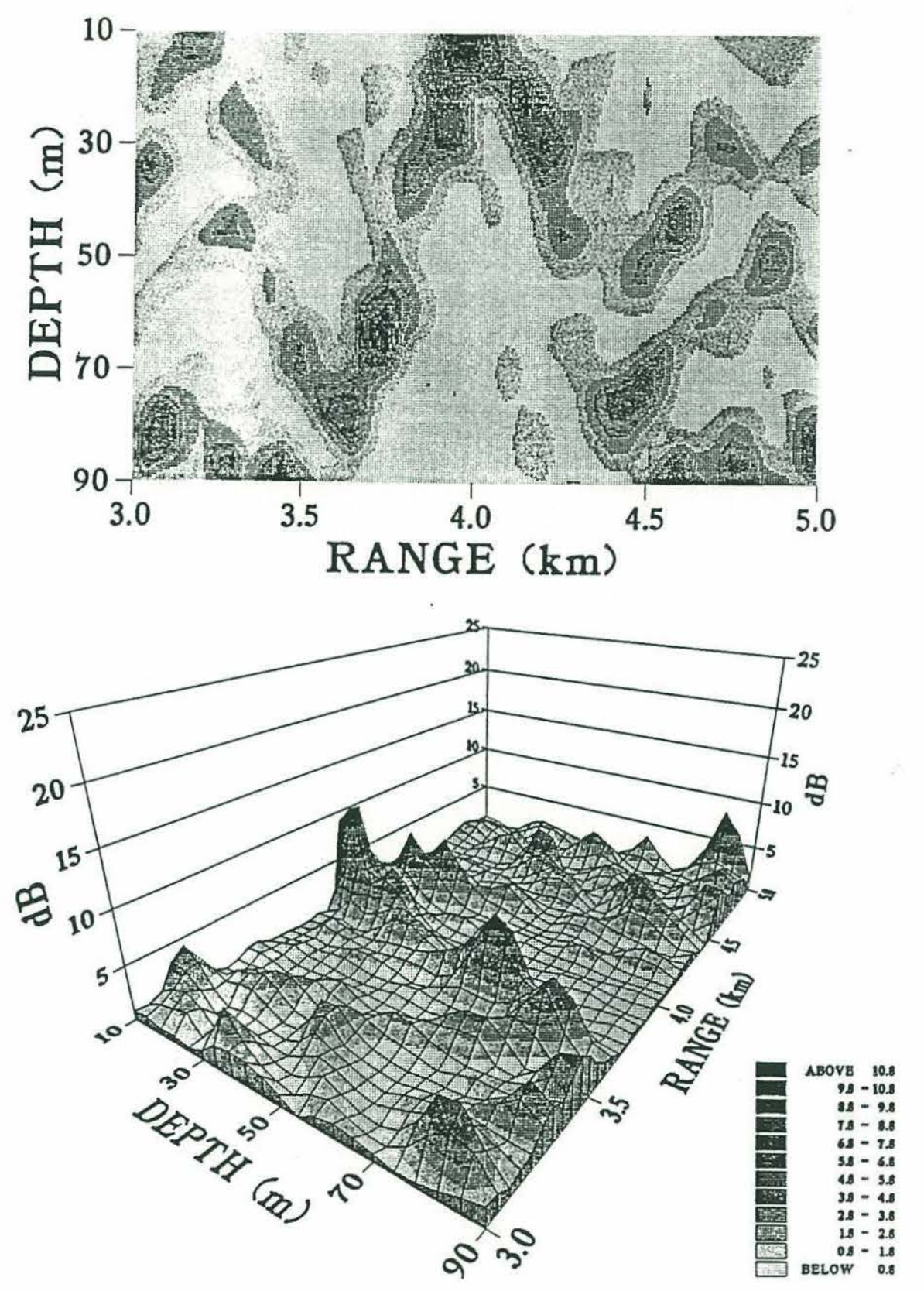

Figure 3-14: MLM ambiguity function for combined effects. True source location is at a range of $4 \mathrm{~km}$ and depth of $14 \mathrm{~m}$ at $\mathrm{t}=0.0$, speed $5 \mathrm{~km} / \mathrm{hr}$ toward receiver. 


\section{Combined Effects (MCM)}
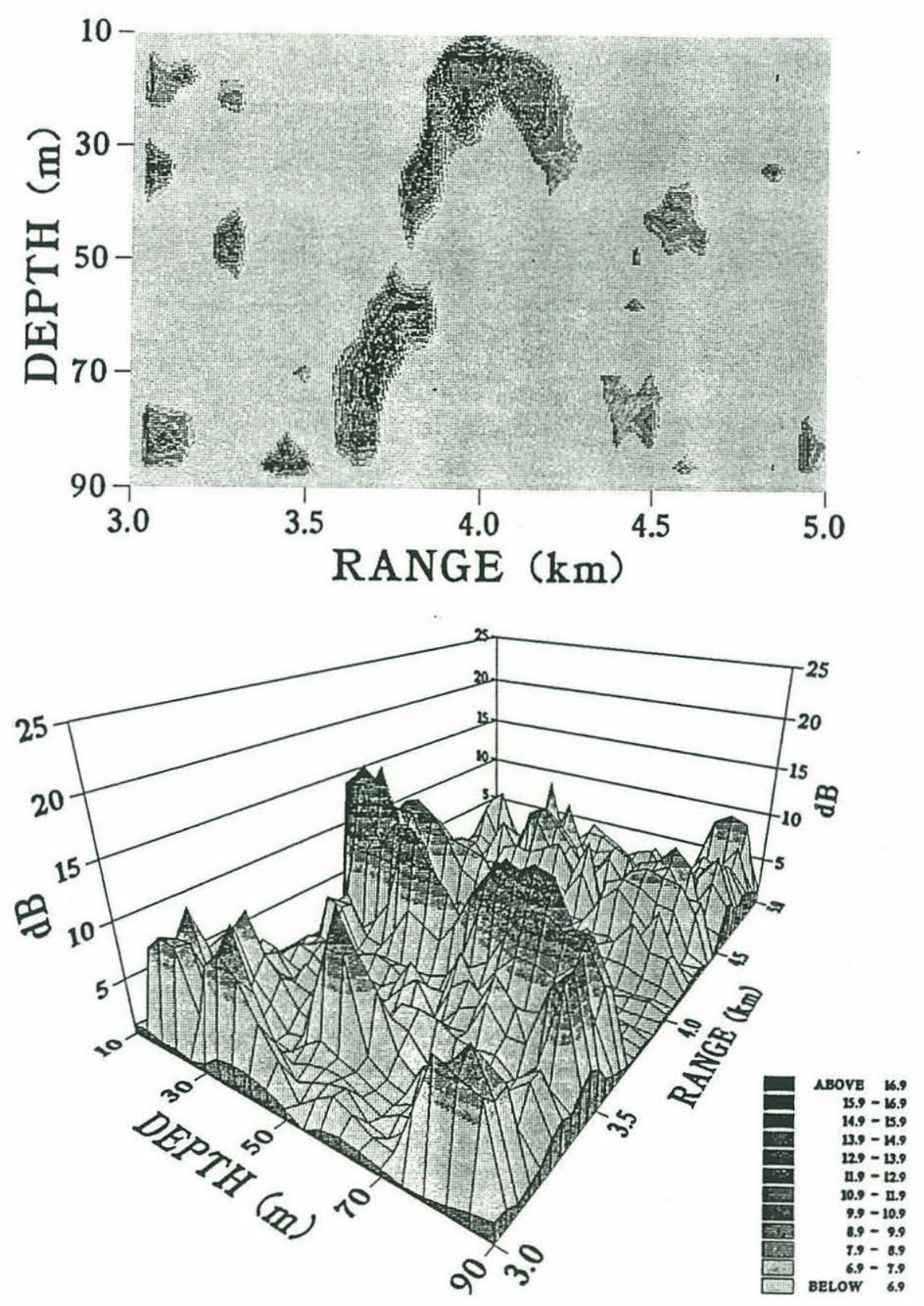

Figure 3-15: MCM ambiguity function for combined effects. True source location is at a range of $4 \mathrm{~km}$ and depth of $14 \mathrm{~m}$ at $\mathrm{t}=0.0$, speed $5 \mathrm{~km} / \mathrm{hr}$ toward receiver. 


\section{Combined Effects (VCLM)}
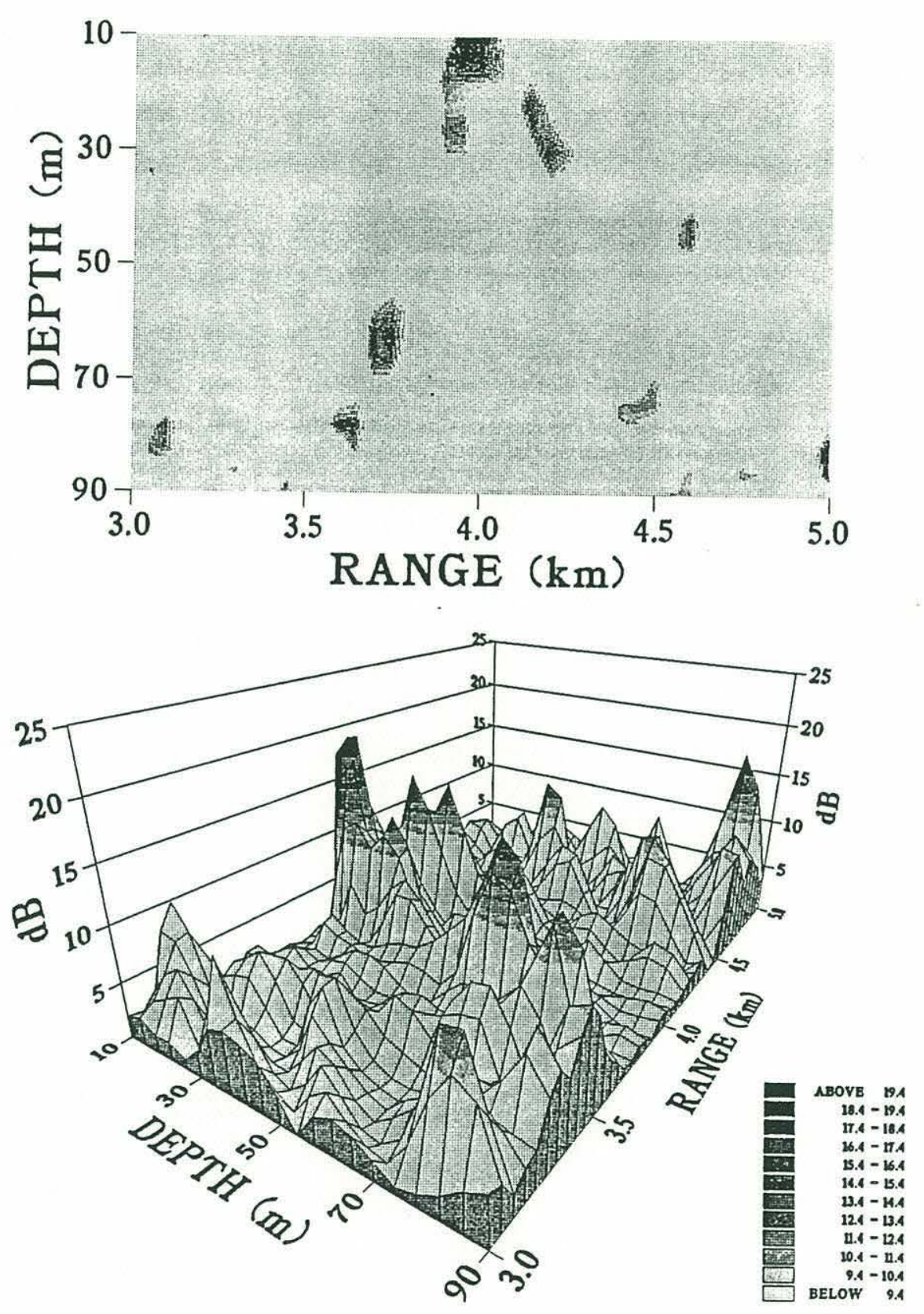

Figure 3-16: VCLM ambiguity function for combined effects. True source location is at a range of $4 \mathrm{~km}$ and depth of $14 \mathrm{~m}$ at $\mathrm{t}=0.0$, speed $5 \mathrm{~km} / \mathrm{hr}$ toward receiver. 


\section{Chapter 4}

\section{Discussion and Conclusions}

Matched field processing has been shown in recent years to be a promising signal processing technique. To date, the Maximum Likelihood Method of parameter estimation has been used most extensively in this field. However, the MLM processor has shown itself to be highly susceptible to errors in the environmental model. In this thesis, we have looked at three such effects; surface roughness, internal waves, and source motion.

Sea surface roughness resulted in a large reduction of the source peak level when using a flat surface model to generate the replica fields. However, if a model is used that includes a surface reflection coefficient based on the statistics of the sea surface, part (but not all) of the signal can be regained. Some signal will be irrecoverable and lead to a reduced PBR which we refer to as the Eckart Limit. The statistic, due to the Gaussian nature of the sea surface, is dependent only upon the variance of the surface roughness and not the actual shape. The variance of the wave height can be observed or, by inverting the problem, estimated from the data. Based on these results, we suggest that in future matched field experiments, random sea surface effects always be included in the model. If using normal mode theory to compute the predicted field, the sea surface statistics can be incorporated as modal attenuation coefficients (Clay,1964; Kuperman and Ingenito,1977).

Internal waves also greatly degraded the ability of matched field processing to perform source localization. Due to the longer wavelengths and slower propagation speeds characteristic of internal waves, the effect is much less statistical in nature than with surface 
waves. The internal wave field must be completely parameterised (amplitude, phase, and phase speed) in order to counteract its effects. As such, we feel internal waves to be a limiting constraint in matched field processing.

Due to the high resolution of the MLM processor in all spatial dimensions, a moving source limits the time period allowed for estimation of the covariance matrix. An extended sampling period allows the source to move through several grid cells and therefore prevents a perfect match to any single point, which results in a degradation of the estimate. On the other hand, by limiting the sample period to a single resolution cell, it is difficult to get a resonable estimate of the covariance matrix, which also degrades the estimate. Thus, for fast moving sources, the two constraints may overlap such that there is no sufficient sampling period which allows adequate localization. The use of an MCM processor (i.e., using an expanded grid area which is stretched in the direction of expected motion) would seem to have some promise in solving this problem and should be investigated.

When confronted with internal waves, the MLM processor lacks the robustness necessary to adequately locate a moving source. Future research should be devoted to the development of a more robust processor instead of further examples highlighting the deficiencies of the MLM processor. We have begun this process by introducing two such processors; the MCM processor being developed by Baggeroer and Schmidt and the VCLM processor suggested here. In the examples shown, the MCM processor showed excellent results for plane wave beamforming but was less successful in localizing a source in a waveguide. This should in no way be construed as a failure of the method. Baggeroer and Schmidt have shown excellent results using different senerios. But it highlights the fact that the method is very dependent upon a combination of grid spacing and the variability of the model field. As a second option, the VCLM processor was introduced by the author. The VCLM processor was found to have increased robustness to mismatch while maintaining very good sidelobe suppression. 


\section{Appendix A}

\section{Derivation of the Surface Scattering Integral}

We derive here the Helmholtz integral equation which we use to calculate the scattering from a rough sea surface. Our development closely follows Eckart's formulation (Eckart,1953; Clay and Medwin, 1977).

\section{A.1 Helmholtz Integral Equation}

Consider a volume $V$ is bounded by the surface $S$ as shown in Figure A-1. Let vector A be a continuous function in the volume. The divergence theorem states that

$$
\iiint_{V} \nabla \cdot \mathbf{A} d v=\iint_{S} \mathbf{A} \cdot \mathbf{n} d s
$$

Let $\mathbf{A}=\phi \nabla G$ where $\phi$ and $G$ are scalar functions. Substituting into Equation (A.1), we have

$$
\iiint_{V}\left(\nabla \phi \cdot \nabla G+\phi \nabla^{2} G\right) d v=\iint_{S} \phi \frac{\partial G}{\partial n} d s
$$

If we let $\mathrm{A}=G \nabla \phi$, repeat the steps and subtract from Equation (A.2), we obtain

$$
\iiint_{V}\left(\phi \nabla^{2} G-G \nabla^{2} \phi\right) d v=\iint_{S}\left(\phi \frac{\partial G}{\partial n}-G \frac{\partial \phi}{\partial n}\right) d s
$$

Let the scalar values of $G$ and $\phi$ be given by

$$
G=\frac{e^{-i k r}}{r} \text { and } \nabla^{2} \phi+k^{2} \phi=0
$$




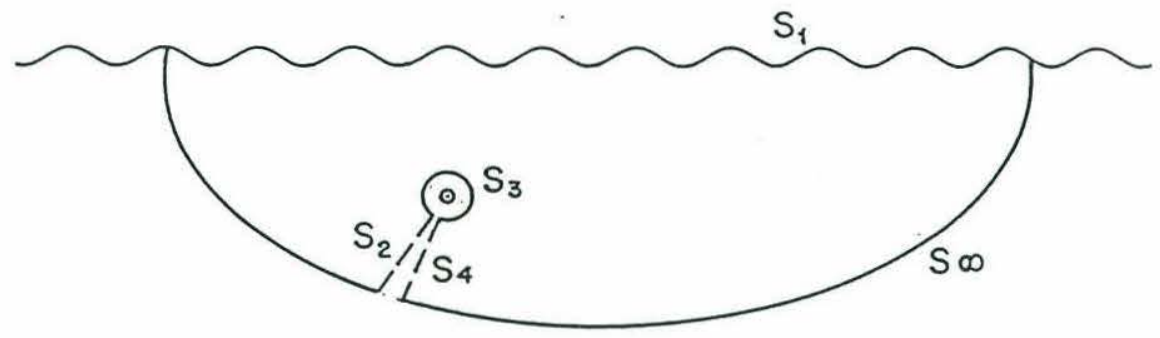

Figure A-1: Calculation of pressure field in a volume V.

We know that the volume contains no sources so that the LHS of Equation (A.3) is equal to zero. Furthermore, the surface integral along the boundary $S_{\infty}$ is zero. Finally, the value of the integration along $S_{2}$ is exactly canceled by the integral along $S_{4}$, leaving us with

$$
-\iint_{S_{3}} \phi \frac{\partial}{\partial n}\left(\frac{e^{-i k r}}{r}\right)-\frac{e^{-i 2 k r}}{r} \frac{\partial}{\partial n}(\phi) d S_{3}=\iint_{S_{1}} \phi \frac{\partial}{\partial n}\left(\frac{e^{-i k r}}{r}\right)-\frac{e^{-i 2 k r}}{r} \frac{\partial}{\partial n}(\phi) d S_{1}
$$

The LHS of Equation (A.5) can be evaluated by taking the limit as $r$ goes to zero. This gives a result which is equal to $4 \pi \phi$, where $\phi$ is evaluated inside the volume $S_{3}$. If the scalar quantity $\phi$ is assumed to be the pressure field, then the pressure at any point $P_{r}$ can be derived by evaluating the pressure field $P_{s}$ along the surface $S_{1}$. This is the Helmholtz integral equation

$$
P_{r}=1 / 4 \pi \iint_{S} P_{s} \frac{\partial}{\partial n}\left(\frac{e^{-i k r}}{r}\right)-\frac{e^{-i 2 k r}}{r} \frac{\partial}{\partial n}\left(P_{s}\right) d s
$$

\section{A.2 Evaluation of Helmholtz Integral Equation}

Equation (A.6) is easily adapted to scattering theory for the case shown in Figure A-2. We assume $P_{s}$ is the reflection of the incident field $P_{i}$. Designating $\Re$ as the reflection 


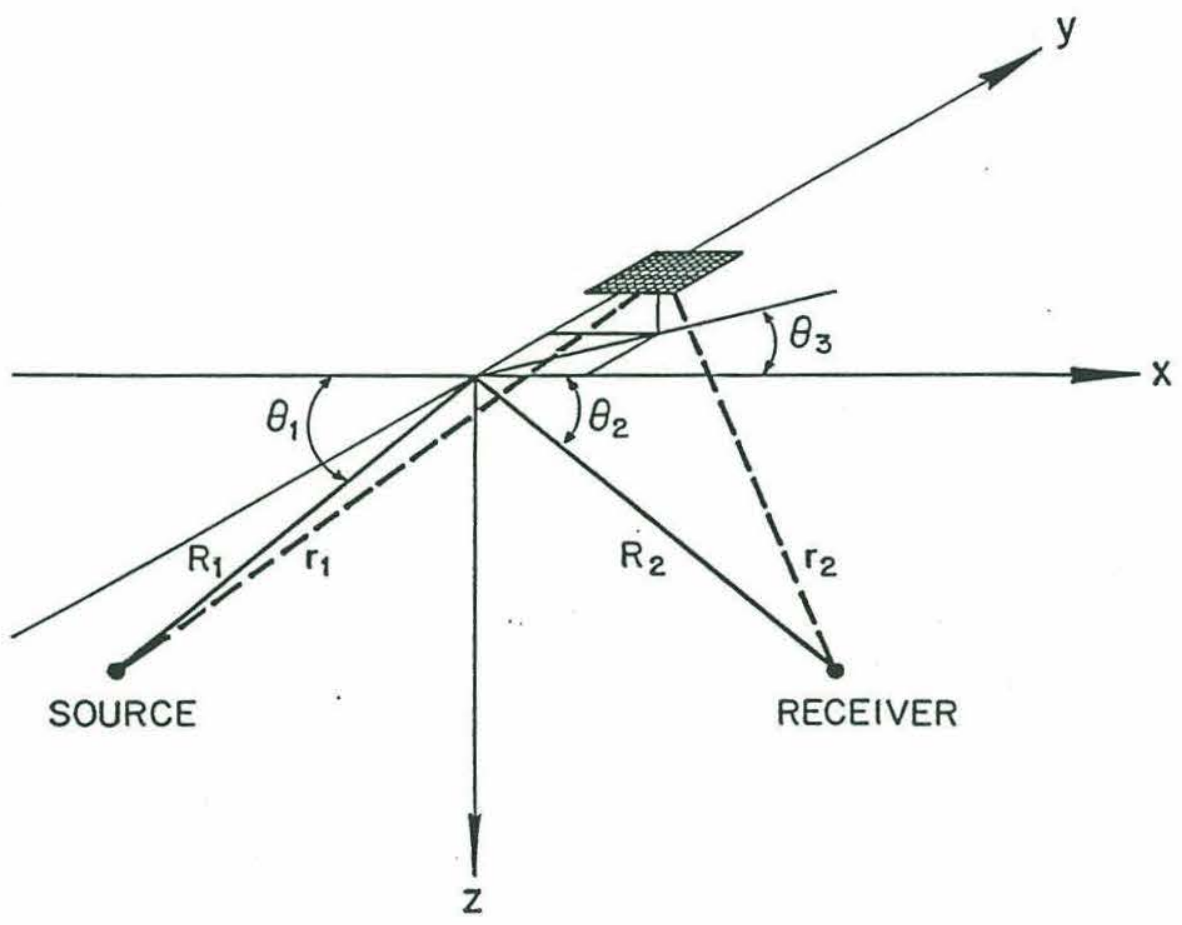

Figure A-2: Scattering from a rough surface 
coefficient, we can write

$$
P_{s}=\Re P_{i} \text { and } \frac{\partial P_{s}}{\partial n}=-\Re \frac{\partial P_{i}}{\partial n}
$$

Substituting into Equation (A.6) gives

$$
P_{r}=1 / 4 \pi \int_{S} \Re \frac{\partial}{\partial n}\left(P_{i} \frac{e^{-i k r_{2}}}{r_{2}}\right) d s
$$

The Kirchhoff approximation assumes that the coefficient $\Re$, which is derived from an infinite plane wave at an infinite plane interface, can be used at every point of the rough surface. At the air water interface, the density contrast is so large that $\Re=-1$ and nearly constant, therefore allowing us to remove $\Re$ from the integral. Futhermore, assuming the incident wave field can be written $P_{0} \frac{e^{-i k r_{1}}}{r_{1}}$, we are left with

$$
P_{r}=\Re / 4 \pi \int_{S} P_{o} \frac{\partial}{\partial n}\left(\frac{e^{-i k\left(r_{1}+r_{2}\right)}}{r_{1}+r_{2}}\right) d s
$$

The rough surface, $\xi$, is a function of $x, y$,and $t$. We now expand the path length into a Taylor series,

$$
k\left(r_{1}+r_{2}\right)=k\left(R_{1}+R_{2}\right)+2(\alpha x+\beta y+\gamma \xi)+x^{2} / x_{f}^{2}+y^{2} / y_{f}^{2}
$$

where

$$
\begin{aligned}
2 \alpha & =k\left(\cos \theta_{1}-\cos \theta_{2} \cos \theta_{3}\right) \\
2 \beta & =-k\left(\cos \theta_{2} \sin \theta_{3}\right) \\
2 \gamma & =-k\left(\sin \theta_{1}+\sin \theta_{2}\right) \\
x_{f}^{-2} & =k / 2\left(\frac{\sin ^{2} \theta_{1}}{R_{1}}+\frac{1-\cos ^{2} \theta_{2} \cos ^{2} \theta_{3}}{R_{2}}\right) \\
y_{f}^{-2} & =k / 2\left(\frac{1}{R_{1}}+\frac{1-\cos ^{2} \theta_{2} \sin ^{2} \theta_{3}}{R_{2}}\right)
\end{aligned}
$$

Assuming a small slope, we approximate $\frac{\partial}{\partial n}$ by $\frac{\partial}{\partial \xi}$. Secondly, we assume $R_{1}$ and $R_{2}$ to be much greater than the perturbation. We are left with

$$
P_{r}=\frac{-i \Re k f(\theta) e^{-i k\left(R_{1}+R_{2}\right)} P_{o}}{2 \pi R_{1} R_{2}} \times \underbrace{\iint_{x=\infty}^{\infty} e^{-i\left(x^{2} / x_{f}^{2}+y^{2} / y_{f}^{2}\right)} d x d y}_{\text {Ilumination Function }} \times \underbrace{\iint_{x=\infty}^{\infty} e^{-i(2 \alpha x+2 \beta y+2 \gamma \xi)} d x d y}_{\text {Roughness Function }}
$$


Given that we are interested only in the specular reflection, we can assume $\theta_{1} \approx \theta_{2}$ and $\theta_{3} \approx 0$, which when substituted into Equation (A.10) gives

$$
\begin{aligned}
2 \alpha & \approx 0 \\
2 \beta & \approx 0 \\
2 \gamma & \approx-2 k \sin \theta_{1} \\
x_{f}^{-2} & \approx k / 2 \sin ^{2} \theta_{1}\left(\frac{R_{1}+R_{2}}{R_{1} R_{2}}\right) \\
y_{f}^{-2} & \approx k / 2\left(\frac{R_{1}+R_{2}}{R_{1} R_{2}}\right)
\end{aligned}
$$

Using the stationary phase approximation which states,

$$
\int_{x} e^{i \phi(x)} d x \simeq e^{i\left(\phi\left(x_{0}\right) \pm \pi / 4\right)} \frac{\sqrt{2 \pi}}{\sqrt{\phi^{\prime \prime}\left(x_{o}\right)}}
$$

the Ilumination function can be approximated as,

$$
\iint_{x=-\infty}^{\infty} e^{-i\left(x^{2} / x_{f}^{2}+y^{2} / y_{f}^{2}\right)} d x d y \simeq i \underbrace{\frac{2 \pi R_{1} R_{2}}{k \sin \theta_{1}\left(R_{1}+R_{2}\right)}}_{=\pi R_{I}^{2}}
$$

We refer to the term $R_{I}$ as the illumination radius, since any ray falling inside the radius is assumed to have an intensity equal to one and any ray incident outside the radius, is asumed to be zero. Substitution into Equation (A.11) gives the final simplified pressure

$$
P_{r}=\frac{\Re P_{o} e^{-i k\left(R_{1}+R_{2}\right)}}{R_{1}+R_{2}} \iint_{\pi R_{I}^{2}} e^{-i 2 \gamma \xi} d A
$$




\section{Bibliography}

Apel, J.R., 1987. Principles of Ocean Physics. Academic Press, Boston.

Baggeroer, A.B., W.A. Kuperman and H. Schmidt, 1988. "Matched field processing: Source localization in correlated noise as an optimum parameter estimation problem". J. Acoust. Soc. Am., 83(2): 571-587.

Born, M. and E. Wolf, 1959. Principles of Optics. Pergamon Press, New York.

Brekhovskikh, L. M., 1960. Waves in Layered Media. Academic Press, New York.

Capon, J., 1969. "High-resolution frequency-wavenumber spectrum analysis". Proc. IEEE, $57: 1408-1418$.

Clay, C.S., 1964. "Effect of a slightly irregular boundary on the coherence of waveguide propogation". J. Acoust. Soc. Am., 36:833-837.

Clay, C. S. and H. Medwin, 1977. Acoustical Oceanography:Principles and Applications. John Wiley and Sons, Inc., New York.

DelBalzo, D.R., C. Feuillade, M.M. Rowe, 1988. "Effects of water-depth mismatch on matched field localization in shallow water". J. Acoust. Soc. Am., 83(6):21802185 .

Dongarra, J.J., C.B. Moler, J.R. Bunch, and G.W. Stewart, 1979. LINPACK User's Guide. SIAM, Philadelphia.

Eckart, C., 1953. "The scattering of sound from the sea surface". J. Acoust. Soc. Am., 25(3): 566-570. 
Feuillade, C., D.R. DelBalzo and M.M. Rowe, 1989. "Environmental mismatch in shallow-water matched-field processing: Geoacoustic parameter variability". $J$. Acoust. Soc. Am., 85(6): 2354-2364.

Fizell, R.G., 1987. "Application of high-resolution processing to range and depth estimation using ambiguity function methods". J. Acoust. Soc. Am., 82(2):606-613. Harris, F.J., 1978. "On the use of windows for harmonic analysis with the discrete Fourier transform". IEEE., 66(1): 51-83.

Hinich, M.J.,1973. "Maximum-likelihood signal processing for a vertical array". J. Acoust. Soc. Am., 54:499-503.

Jenson, F.B. and H. Schmidt, 1987. "Subcritical penetration of narrow Gaussian beams into sediments". J. Acoust. Soc. Am., 82(2): 574-579.

Katz, E.J., 1966. "Effect of the propagation of internal water waves on underwater sound transmission". J. Acoust. Soc. Am., 42(1): 83-87.

Kay, S.M. and S.L. Marple, Jr., 1981. "Spectrum analysis - a modern perspective". IEEE., 69(11): 1380-1419.

Kuperman W.A. and F. Ingenito, 1977. "Attenuation of the coherent component of sound propagating in shallow water with rough boundries". J. Acoust. Soc. Am., 61: 1178-1187.

Lee, O.S., 1961. "Effect an internal wave on sound in the ocean". J. Acoust. Soc. Am., 33(5): 677-681.

McCammon, D.F., 1984. "An Evaluation of the Kirchhoff Approximation for Acoustic Plane Wave Reflection Coefficients from a Sinusoidal Boundary". Doctoral Thesis, The Pennsylvania State University.

Orr, M., 1980. "Remote acoustic sensing of the oceanic fluid and biological processes". Woods Hole Oceanographic Institute Technical Report, WHOI-80-2. 
Pierson, W.J., Jr., and R.A. Stacy, 1973. "The elevation, slope, and curvature spectrum of a wind roughened sea surface", NASA Contractor Rep. CR-2247.

Schmidt, H., 1987. "SARARI. seismo - acoustic fast field algorithm for range independent environments, user's guide". SACLANT ASW Research Centre, la Spezia, Italy, Rep. SR.

Schmidt, H. and F.B. Jensen, 1985. "A full wave solution for propagation in multilayered visoelastic media with application to Gaussian beam reflection of fluid solid interfaces". J. Acoust. Soc. Am., 77: 813-825.

Tindle, C.T. and E.J. Bold, 1981. "Improved ray calculations in shallow water". $J$. Acoust. Soc. Am., 70(3): 813-819.

Tindle, C.T., 1983. "Ray calculations with beam displacements". J. Acoust. Soc. Am., 73(5): 1581-1586.

Tolstoy, A., 1989. "Sensitivity of matched field processing to sound- speed profile mismatch for vertical arrays in a deep water Pacific environment". J. Acoust. Soc. Am., 85(6): 2394-2404.

Tung C.C. and N.E. Huang., 1985. "Covariances and spectra of the kinematics and dynamics of nonlinear waves". J. Geophys. Res., 90(C6):11911-11916.

Van Trees, H. C., 1968. Detection, Estimation, and Modulation Theory, Part I. Wiley, New York.

Westwood, E.K. and C.T.Tindle, 1987. "Shallow water time-series simulation using ray theory". J. Acoust. Soc. Am., 81(6): 1752-1761.

Zhang R., 1975. "The normal mode sound field in a shallow water surface sound field". Acta. Phys. Sin., 24: 200-209. 